Aus der Abteilung Kardiologie und Pneumologie

(Prof. Dr. med. G. Hasenfuß)

im Zentrum Innere Medizin

der Medizinischen Fakultät der Universität Göttingen

\title{
Einfluss von typischen Komorbiditäten auf die Ausprägung der Symptomatik bei Herzinsuffizienz mit eingeschränkter und erhaltener linksventrikulärer Funktion
}

\section{INAUGURAL - DISSERTATION}

zur Erlangung des Doktorgrades

der Medizinischen Fakultät

der Georg-August-Universität zu Göttingen

vorgelegt von

Kathleen Durstewitz

aus

Heiligenstadt 
Dekan: Prof. Dr. med. C. Frömmel

I. Berichterstatter: PD Dr. med. R. Wachter

II. Berichterstatter: Prof. Dr. rer. nat. R. Hilgers

Tag der mündlichen Prüfung: 04. Dezember 2012 


\section{Inhalt}

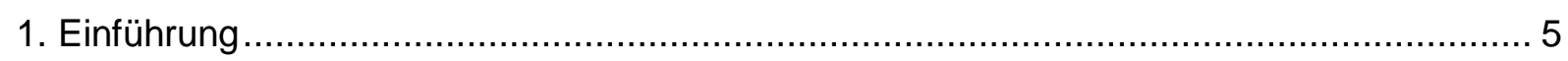

1.1 Definition und Klinik der chronischen Herzinsuffizienz …................................... 5

1.2 Ätiologie und Epidemiologie der chronischen Herzinsuffizienz.............................. 7

1.3 Therapie der chronischen Herzinsuffizienz ................................................... 8

1.3.1 Medikamentöse Therapie chronischer Herzinsuffizienz mit eingeschränkter

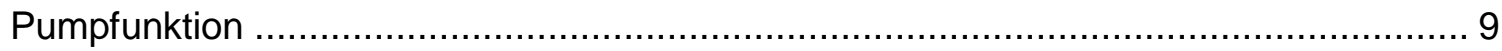

1.3.2 Medikamentöse Therapie chronischer Herzinsuffizienz mit erhaltener

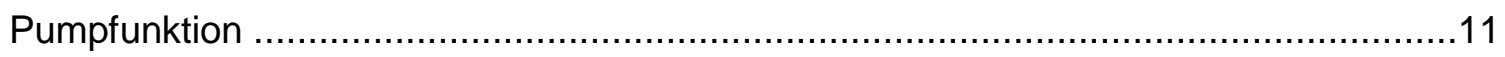

1.4 Stellenwert der Komorbiditäten bei chronischer Herzinsuffizienz ............................14

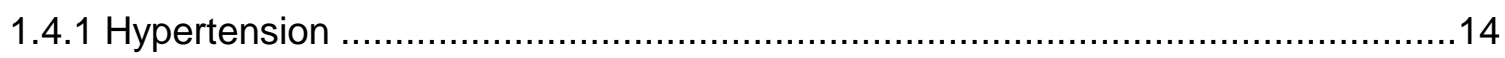

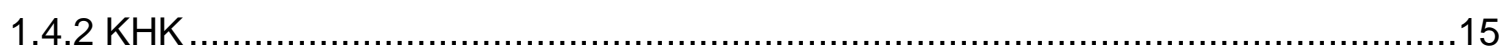

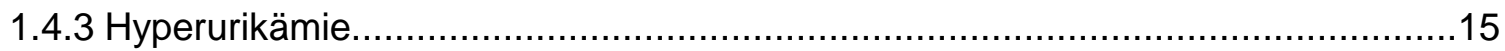

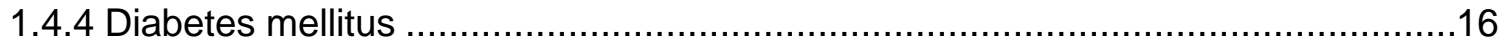

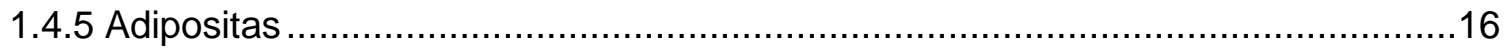

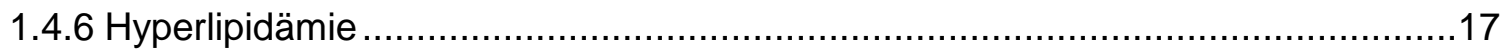

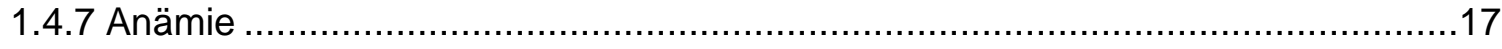

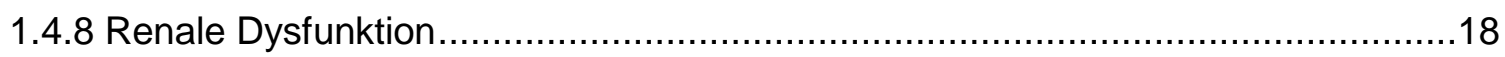

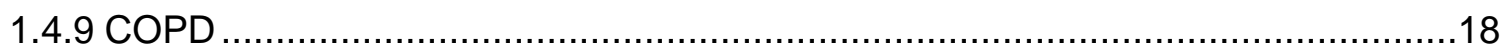

1.5 Fragestellung und Zielsetzung der vorliegenden Arbeit .......................................19

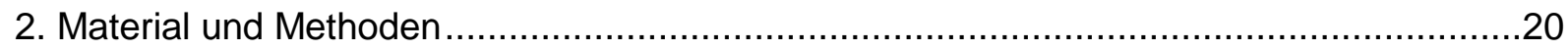

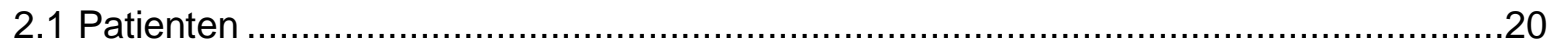

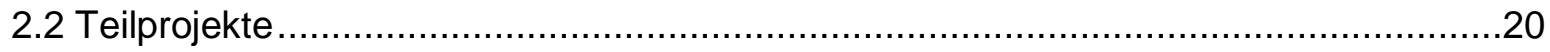

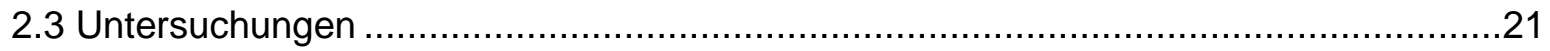

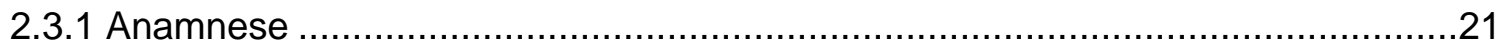

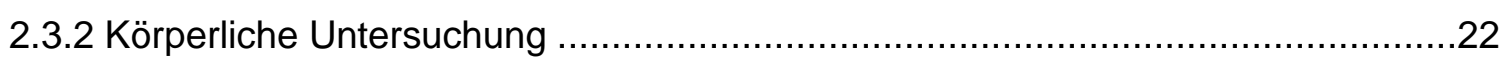

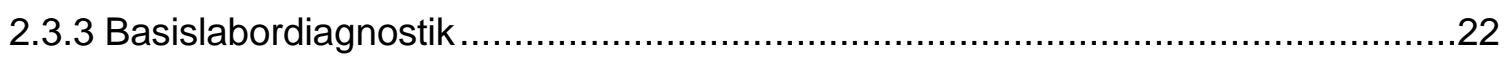

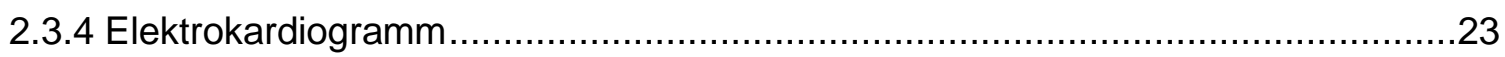

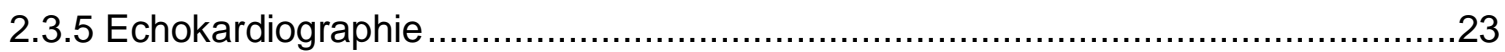

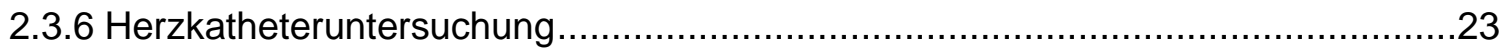

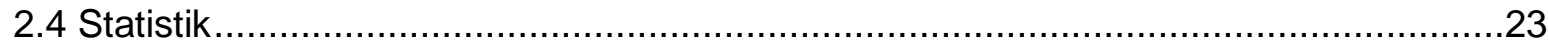

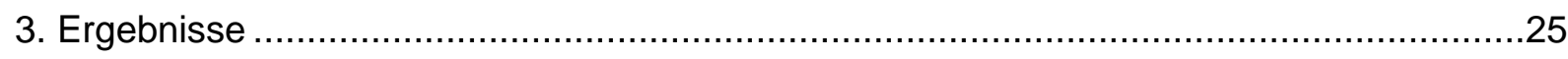

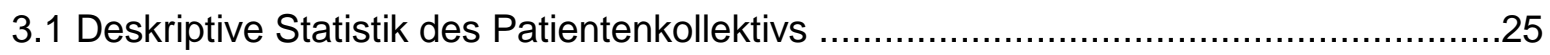

3.1.1 Patienten und wissenschaftliche Projekte ...................................................25

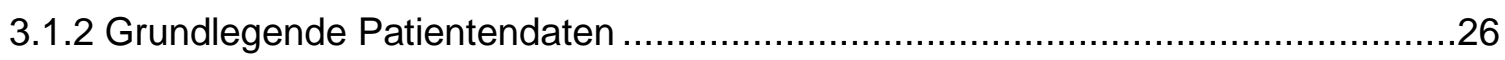

3.1.3 Zeichen und Symptome der Herzinsuffizienz im Patientenkollektiv ......................27 


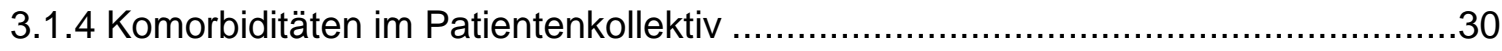

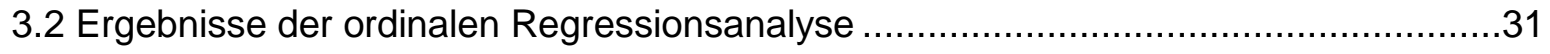

3.3 Ergebnisse der multivariaten Analyse bei HFREF und HFPEF .............................43

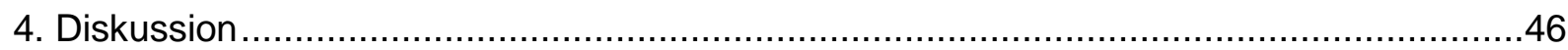

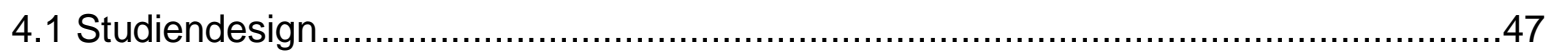

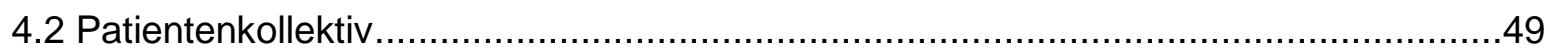

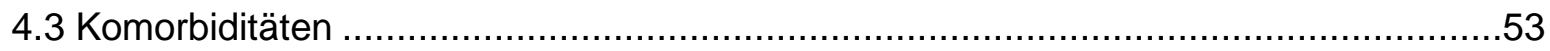

4.3.1 Verteilung der Komorbiditäten bei HFREF und HFPEF ....................................53

4.3.2 Verhältnis/Einfluss pathophysiologischer Kenngrößen und Komorbiditäten auf die

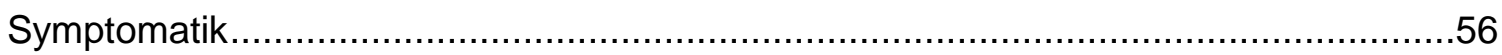

4.3.2.1 Gleichgerichtete Interaktionen von Komorbiditäten und Symptomatik bei

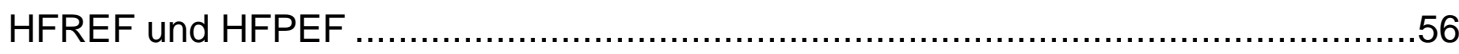

4.3.2.2 Konträre Interaktionen von Komorbiditäten und Symptomatik bei HFREF und HFPEF

4.3.2.3 Inverse Interaktionen

68

4.3.3 Der Stellenwert der Komorbiditäten bezüglich der Symptomatik chronisch herzinsuffizienter Patienten im Vergleich zu Parametern der ventrikulären systolischen und diastolischen Funktion ............................................................................ 71

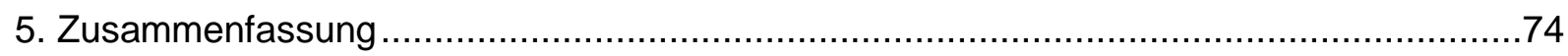

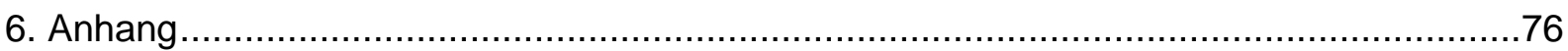

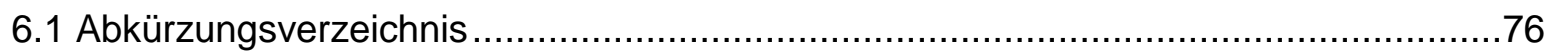

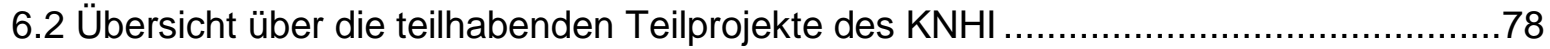

6.3 Beispielhafte Darstellung von Screenshot- und Outputdokumenten.........................98

6.3.1 Ordinale Regressionsanalyse: Screenshot-Dokument am Beispiel von Diabetes

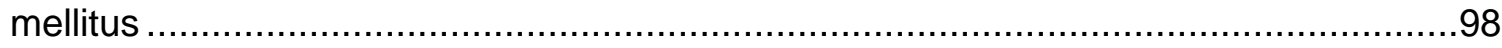

6.3.2 Ordinale Regressionsanalyse: SPSS-Outputdokument am Beispiel von Diabetes

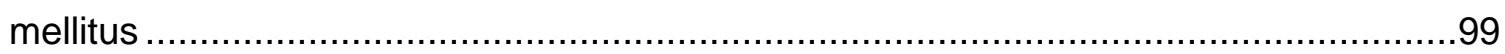

6.3.3 Multivariate Analysen: Screenshot- und Outputdokument.............................103

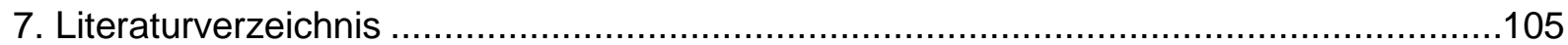




\section{Einführung}

\subsection{Definition und Klinik der chronischen Herzinsuffizienz}

Die Herzinsuffizienz ist ein komplexes Geschehen struktureller und funktioneller Veränderungen des Herzmuskels, wodurch dieser die Fähigkeit verliert, den Organismus mit ausreichend oxigeniertem Blut zu versorgen. Als Multiorgansyndrom ist die Herzinsuffizienz gekennzeichnet durch typische Symptome: Dyspnoe in Ruhe oder unter Belastung, Zeichen der Flüssigkeitsretention (Ödeme, Lungenstauung) und eines objektiven Nachweises vorhandener struktureller und funktioneller Abnormalitäten des Herzens in Ruhe. Das Vorliegen dieser Abnormalitäten in Abwesenheit von Symptomen wird als Vorläufer einer symptomatischen Herzinsuffizienz bezeichnet und geht auch schon mit einer erhöhten Mortalität einher (ESC guidelines 2008).

Anhand der Pathogenese unterscheidet man die chronische Herzinsuffizienz mit erhaltener Ejektionsfraktion (diastolische Herzinsuffizienz) von einer mit reduzierter Ejektionsfraktion (systolische Herzinsuffizienz).

Bei der systolischen Herzinsuffizienz kommt es infolge von Kontraktilitätsschwäche und Ventrikelspannung (verursacht durch Volumen- und/oder Druckbelastung) zu einer Dilatation des Ventrikels, vergrößerten Ventrikelvolumina und verminderter Kontraktilität. Die Ejektionsfraktion ist hier auf $<50 \%$ eingeschränkt. Man spricht von einer HFREF (heart failure with reduced ejection fraction).

Bei 30 bis 50\% der Patienten mit typischen Symptomen einer Herzinsuffizienz liegt der Erkrankung eine primär diastolische Funktionsstörung zugrunde. Diese Patienten sind neben einer Herzinsuffizienzsymptomatik durch eine normale oder nahezu normale systolische Funktion $(E F \geq 50 \%)$ gekennzeichnet. In diesem Fall spricht man von einer HFPEF (heart failure with preserved ejection fraction). Eine verzögerte Relaxation, zunehmende Kammersteifigkeit und eine verminderte diastolische Dehnbarkeit und Füllung können ebenfalls nachgewiesen werden. Wenn diese Diastolische Dysfunktion nicht nachgewiesen wird, spricht man von HFNEF (heart failure with normal ejection fraction, deutsch: Herzinsuffizienz mit normaler Ejektionsfraktion).

International erfolgt die Einteilung der Herzinsuffizienz nach der New-York-Heart-Association (NYHA)- Klassifikation. Hier wird die Schwere der Herzinsuffizienz, basierend auf Symptomatik und Leistungsfähigkeit der Patienten, in vier Stadien eingeteilt:

NYHA I Völlige Beschwerdefreiheit bei normaler körperlicher Belastung

NYHA II geringe Einschränkung der körperlichen Leistungsfähigkeit bei normaler Belastung, Dyspnoe beim schnelleren Gehen oder Laufen 
NYHA III starke Einschränkung der körperlichen Leistungsfähigkeit bei normaler Belastung, Dyspnoe beim Treppensteigen oder längerem Gehen zu ebener Erde

NYHA IV Einschränkung der körperlichen Leistungsfähigkeit selbst bei geringer Belastung oder bereits in Ruhe, Dyspnoe bereits nach kurzen Wegstrecken oder in Ruhe.

Eine weitere Einteilung anhand von Symptomen ermöglicht die Framingham-Klassifikation. Hier wurden manifeste Symptome und klinische Zeichen in einem CHF (congestive heart failure)- Score zusammengefasst, der nach Haupt- und Nebenkriterien unterscheidet: Sind zwei Hauptkriterien oder ein Haupt- und zwei Nebenkriterien bei einer Person vorhanden, so wird die Diagnose einer Herzinsuffizienz gestellt.

Symptome der Herzinsuffizienz äußern sich in Dyspnoe, Orthopnoe, pathologischer Ermüdung und reduzierter körperlicher Leistungsfähigkeit, Gewichtszunahme und Nykturie. Zusätzlich zu den aufgeführten Symptomen kann man bei der klinischen Untersuchung für die Herzinsuffizienz folgende Zeichen finden: Halsvenenstauung, einen positiven hepatojugulären Reflux, einen verbreiterten Herzspitzenstoß, einen dritten Herzton, feinblasige Rasselgeräusche, Pleura- oder Perikardergüsse, periphere Ödeme, Aszites, Hepatomegalie, Tachykardie, Hyperkapnie und kardiale Kachexie (ESC guidelines 2008).

Diagnostiziert wird eine Herzinsuffizienz beim Auftreten einzelner oder aller genannten subjektiven und klinischen Zeichen. Neben einer ausführlichen Anamnese, körperlicher Untersuchung, Durchführung eines Elektrokardiogramms und Erhebung von Laborparametern werden bildgebende Verfahren eingesetzt. Der Goldstandard der bildgebenden Herzinsuffizienzdiagnostik ist die Echokardiographie. Zusätzlich erfolgt der Einsatz von Röntgen-Thorax und Abdomen-Sonographie. Aufwendig ist die Durchführung einer Herzkatheteruntersuchung, die eher dem Ausschluss zugrundeliegender Ursachen wie KHK und Herzklappenfehler dient. Ein anerkanntes Verfahren zur Beurteilung der körperlichen Leistungsfähigkeit herzinsuffizienter Patienten ist die Spiroergometrie, welche unter anderem Herzfrequenz, Blutdruck, Atemvolumina, Atemfrequenz, maximale Sauerstoffaufnahme und die anaerobe Schwelle unter Belastung ermittelt. Der SechsMinuten-Geh-Test ist eine kostengünstige Alternative und ermöglicht die Erhebung der subjektiven Belastbarkeit der Patienten mit chronischer Herzinsuffizienz (ESC guidelines 2008). 


\section{2 Ätiologie und Epidemiologie der chronischen Herzinsuffizienz}

Die häufigsten bekannten Ursachen, die zu der Entstehung einer Herzinsuffizienz führen können, sind der Verlust und die Beschädigung des Herzmuskels, akute und chronische Ischämie, ein steigender vaskulärer Widerstand mit Hypertonie und die Ausbildung von Tachyarrhythmien (z.B. Vorhofflimmern). Die koronare Herzerkrankung ist die häufigste Ursache einer myokardialen Erkrankung und stellt bei 70\% der Patienten den Auslöser einer Herzinsuffizienz dar. Der Anteil der Klappenerkrankungen beträgt 10\% und die Kardiomyopathien machen weitere $10 \%$ aus (ESC Guidelines 2008).

Herzinsuffiziente Patienten mit erhaltener Pumpfunktion weisen neben einer altersbedingten Compliancestörung des Ventrikels als häufigste Ursache die Hypertrophie infolge eines arteriellen Hypertonus auf. Bei Patienten mit eingeschränkter EF wird die Herzinsuffizienz zum einen häufig durch einen Hypertonus und zum anderen durch die ischämische Herzerkrankung in Form des akuten Koronarsyndroms oder als chronische Form der Koronararterienstenose verursacht (Diller et al. 1999).

Nach jährlichen Schätzungen variiert die Inzidenz der Herzinsuffizienz bei 45- bis 74-jährigen Frauen von 0,9 bis 2,2 pro 1000 der Bevölkerung und bei 45- bis 74-jährigen Männern von 1,6 bis 4,6 pro 1000. Die Inzidenz wächst rapide mit steigendem Alter. Das männliche Geschlecht ist dabei insgesamt häufiger betroffen (m:w 1,5:1). Pro Jahr entwickeln 1\% der >75-jährigen Männer eine Herzinsuffizienz und diese Rate steigt bis zu 2\% bei den über 80jährigen Männern (Hobbs 2009).

Die Prävalenz der chronischen Herzinsuffizienz beträgt 2 bis 3\%, steigt deutlich bis zum 75 . Lebensjahr und beträgt bei 70 - bis 80 -Jährigen 10 bis $20 \%$. Die Herzinsuffizienz mit erhaltener Pumpfunktion tritt etwa bei der Hälfte der Patientenpopulation mit chronischer Herzinsuffizienz auf und kommt häufiger im fortgeschrittenen Alter und beim weiblichen Geschlecht vor (ESC Guidelines 2008).

Owan et al. untersuchten die Prävalenz der Herzinsuffizienz mit erhaltener Pumpfunktion über eine 15-jährige Periode (1987-2001). In diesem Zeitraum konnte eine Zunahme der Prävalenz einer HFPEF von 38 auf 54\% gezeigt werden. Der Anstieg entstand durch wachsende Krankenhauseinweisungen herzinsuffizienter Patienten mit erhaltener Pumpfunktion, während die Zahl der Patienten mit reduzierter Ejektionsfraktion über diesen Zeitraum unverändert blieb (Owan et al. 2006).

Die Morbidität der chronischen Herzinsuffizienz ist sehr hoch und resultiert aus der Schwere der Symptome, der Lebensqualität und der Hospitalisierungsrate. Es existieren nur wenige 
Studien, die aber belegen, dass die Herzinsuffizienz größere Auswirkungen auf die Lebensqualität hat als andere chronische Erkrankungen. Ebenfalls wurde bewiesen, dass Herzinsuffizienz mit depressiven Erkrankungen und einer schlechteren Prognose assoziiert ist (Hobbs 2009). Die Morbiditätsrate bei wegen Herzinsuffizienz hospitalisierter Patienten mit reduzierter Ejektionsfraktion ist nahezu identisch mit derjenigen mit erhaltener EF (Owan et al. 2006).

Die jährliche Mortalität für Patienten mit Herzinsuffizienz und eingeschränkter Pumpfunktion beträgt annährend 10 bis 15\%. Im Vergleich dazu liegt diese bei Patienten mit erhaltener EF bei 5 bis 8\%, während die dem Alter entsprechende Kontrollgruppe eine jährliche Mortalitätsrate von 1\% aufweist. Bei herzinsuffizienten Patienten von über 70 Jahren sind die Mortalitätsraten bei erhaltener und bei eingeschränkter Pumpfunktion nahezu identisch (Zile und Brutsaert 2002 a).

Die Prognose von systolischer und diastolischer Herzinsuffizienz wird in klinischen Studien unterschiedlich beurteilt: Die mittlere Überlebenszeit beträgt bei Patienten mit erhaltener Ejektionsfraktion 7,1 Jahre und 4,3 Jahren bei Patienten mit eingeschränkter Pumpfunktion (Vasan et al. 1999). Owan et al. untersuchten die Veränderungen der Überlebensrate zwischen herzinsuffizienten Patienten mit reduzierter und erhaltener Ejektionsfraktion über ein fünfjähriges Intervall. Die Überlebensrate bei herzinsuffizienten Patienten mit erhaltener $E F$ war höher als bei jenen mit eingeschränkter EF, allerdings nur geringfügig. Die Patienten mit eingeschränkter EF zeigten eine Verbesserung des Überlebens während des fünfjährigen Intervalls, während unter den Patienten mit erhaltener EF kein Verbesserungstrend nachgewiesen werden konnte (Owan et al. 2006). Bei Patienten mit reduzierter Pumpfunktion ist vor allem durch verbesserte therapeutische Strategien eine stetige Verbesserung der Überlebensrate nachweisbar. Für die Patienten mit erhaltener EF sind noch keine derartigen Therapieoptionen bekannt, was das Stagnieren der Überlebensrate begründen könnte (Owan et al. 2006).

Bhatia et al. beobachteten über ein Zeitintervall von einem Jahr keine signifikant höhere Mortalitätsrate bei herzinsuffizienten Patienten mit reduzierter EF im Vergleich zu herzinsuffizienten Patienten mit erhaltener EF (Bhatia et al. 2006).

\subsection{Therapie der chronischen Herzinsuffizienz}

Die Therapie der chronischen Herzinsuffizienz verfolgt grundsätzlich drei Ziele:

1. Prognoseverbesserung (Reduktion der Mortalität)

2. Verbesserung der Morbidität (Symptomlinderung, Verbesserung von Lebensqualität und Leistungsfähigkeit, Reduktion der Hospitalisierungsrate) 
3. Prävention (Verhindern des Auftretens und der Progression von Myokardschäden) (ESC Guidelines 2008).

Um diese Ziele umzusetzen, stehen sowohl medikamentöse als auch nichtmedikamentöse bzw. kausale Therapieoptionen zur Verfügung. Der Idealfall einer kausalen Therapie besteht in der Beseitigung der zugrunde liegenden Ursache. Dies beinhaltet unter anderem eine leitliniengerechte antihypertensive Therapie, Beseitigung und Vermeidung von Ischämien, Korrektur von Vitien, Diagnose und Behandlung eines Schlafapnoe-Syndroms und eine optimale Diabeteseinstellung.

Die nicht medikamentöse Therapie setzt sich zusammen aus:

- Gewichtsnormalisierung und täglicher Gewichtskontrolle durch den Patienten,

- Kochsalzzufuhr $<3 \mathrm{~g} / \mathrm{d}$,

- reduzierte Flüssigkeitszufuhr auf $\leq 2 \mathrm{l} / \mathrm{d}$,

- Reduktion kardiovaskulärer Risikofaktoren (Zigaretten, Alkohol, Cholesterin),

- körperliches Training bei kompensierter Herzinsuffizienz und Schonung/Bettruhe in Phasen der Dekompensation.

Diese Punkte sind nur für die Therapie der systolischen Herzinsuffizienz evidenzbasiert, finden aber eine allgemeine Anwendung in der Therapie der chronischen Herzinsuffizienz (ESC Guidelines 2008).

\subsubsection{Medikamentöse Therapie chronischer Herzinsuffizienz mit eingeschränkter Pumpfunktion}

Die Therapie der chronischen Herzinsuffizienz mit eingeschränkter Pumpfunktion erfolgt durch eine standardisierte, evidenzbasierte medikamentöse Therapie mit ACE-Hemmern, AT1-Rezeptorblockern, Betablockern und Aldosteronantagonisten, die Einfluss auf die Morbidität und Mortalität haben. Diuretika und Digoxin kommen als symptomorientierte Medikamente zum Einsatz, während Antikoagulanzien und HMG-CoA-Reduktasehemmer der Therapie vorhandener kardiovaskulärer Komorbiditäten dienen. Calciumkanalblocker spielen bei der Herzinsuffizienz mit eingeschränkter Pumpfunktion keine Rolle (ESC Guidelines 2008).

Ein ACE-Hemmer sollte bei allen Patienten mit symptomatischer Herzinsuffizienz und einer eingeschränkten linksventrikulärer Ejektionsfraktion (LVEF) verwendet werden. Die Therapie mit ACE- Hemmern verbessert die Ventrikelfunktion und das Wohlbefinden des Patienten, reduziert die Hospitalisierungsrate für fortschreitende Herzinsuffizienz und steigert das Überleben. 
In zwei randomisierten kontrollierten Studien (CONSENSUS und SOLVD treatment) konnte gezeigt werden, dass eine ACE-Hemmer-Therapie die Mortalität reduziert (ESC Guidelines 2008).

Der Einsatz von Betablockern sollte bei allen Patienten mit symptomatischer Herzinsuffizienz (NYHA II-IV) und einer LVEF $\leq 40 \%$ ergänzend zu einer optimalen ACE-Hemmer- und/oder AT1-Rezeptorblocker-Basistherapie, erfolgen. Bei fortgeschrittener Herzinsuffizienz ist eine einschleichende Dosierung indiziert. Betablocker verbessern die Ventrikelfunktion und das Wohlbefinden des Patienten. In drei großen randomisierten, placebokontrollierten Studien (CIBISII, COPERNICUS und MERIT-HF) wurde bewiesen, dass eine Behandlung mit Betablockern (Bisoprolol, Carvedilol oder Metoprololsuccinat) die Mortalität reduziert und die Hospitalisierungsrate bei fortgeschrittener Herzinsuffizienz senkt (ESC Guidelines 2008).

Die zusätzliche Verabreichung eines niedrig dosierten Aldosteronantagonisten (Spironolacton, Eplerenon) sollte bei Patienten mit einer LVEF $\leq 35 \%$ und schwerer Herzinsuffizienzsymptomatik (NYHA III oder IV) in Erwägung gezogen werden, sofern Hyperkaliämie und signifikante renale Dysfunktion ausgeschlossen werden können (ESC Guidelines 2008). In zwei randomisierten placebokontrollierten Studien (RALES und EPHESUS) bewirkten Aldosteronrezeptorantagonisten in Addition zu einer Basistherapie eine Reduktion der Zahl der Krankenhausaufenthalte und verbesserten das Überleben bei Patienten mit schwerer systolischer Herzinsuffizienz sowie bei Herzinsuffizienz infolge eines Myokardinfarktes (Zannad et al. 2011).

Ebenfalls konnte kürzlich in der placebokontrollierten EMPHASIS-HF-Studie eine Reduktion der Sterblichkeit sowie der Hospitalisierung unter der Therapie mit Eplerenon in Addition zur empfohlenen Therapie bei Patienten mit chronischer systolischer Herzinsuffizienz und milder Symptomatik (NYHA II) nachgewiesen werden (Zannad et al. 2011).

AT1-Rezeptorblocker haben gegenüber einer ACE-Hemmer-Therapie keinen prognostischen Vorteil. Sie werden daher bei herzinsuffizienten Patienten mit einer LVEF $\leq 40 \%$ entweder als Therapiealternative bei ACE-Hemmer-Unverträglichkeit oder bei Patienten mit persistierender Symptomatik, trotz ACE-Hemmer- und Betablockertherapie angewandt.

Bei Patienten mit symptomatischer Herzinsuffizienz (NYHA II-IV) und Vorhofflimmern kann Digoxin zur Verlangsamung der ventrikulären Frequenz eingesetzt werden. Für eine Herzfrequenzkontrolle bei Vorhofflimmern und einer LVEF $\leq 40 \%$ sollte Digoxin zusätzlich zu einem Betablocker verabreicht werden. Es konnte keine Mortalitätssenkung, Reduktion der 
Hospitalisierungsrate oder eine Verbesserung des klinischen Endergebnisses unter Digoxintherapie nachgewiesen werden (ESC Guidelines 2008).

Die Behandlung mit Diuretika wird bei Patienten mit Herzinsuffizienz und Zeichen der pulmonalen und systemischen Stauung sowie bei vorhandenen Ödemen und Symptomen der Flüssigkeitsüberladung empfohlen. Die Effekte auf die Prognose sind allerdings noch nicht nachgewiesen. Die Therapie sollte in Kombination mit ACE-Hemmern oder AT1Rezeptorblockern erfolgen (ESC Guidelines 2008).

\subsubsection{Medikamentöse Therapie chronischer Herzinsuffizienz mit erhaltener Pumpfunktion}

Die medikamentöse Therapie der chronischen Herzinsuffizienz mit erhaltener Pumpfunktion ist derzeit noch theoretisch und empirisch. Bisher konnten keine Therapien etabliert werden, die die Morbidität und Mortalität senken (ESC Guidelines 2008).

Deshalb wird dazu empfohlen, die Therapie in drei Stufen aufzubauen:

1. Symptomorientierte Therapie,

2. Behandlung von Grunderkrankungen,

3. Therapie herzinsuffizienzverursachender Mechanismen.

An erster Stelle der Symptomreduktion stehen die Senkung des pulmonalvenösen Drucks und eine Reduktion der Lungenstauung. Dies beinhaltet eine Senkung des LV-Volumens, den Erhalt einer synchronen Vorhofkontraktion und eine Verlängerung der Diastole durch eine Reduktion der Herzfrequenz. Infolge einer Reduktion des LV-diastolischen Volumens fällt auch der LV-diastolische Druck. Der Einsatz von Diuretika und Nitraten sowie eine kontrollierte Salz- und Flüssigkeitszufuhr können das totale und zentrale Blutvolumen reduzieren und damit den linksventrikulären diastolischen Druck senken. Ebenso können Diuretika das Auftreten von Ödemen und die Luftnotsymptomatik lindern.

Eine Aktivierung des neurohumoralen und des Renin-Angiotensin-Aldosteron-Systems führt zu Flüssigkeitsretention und damit zu einem systemischen Volumenanstieg und geht laut klinischen Studien ebenfalls mit der Entstehung von Hypertrophie einher. Daher sollte die Therapie der chronischen Herzinsuffizienz bei erhaltener Pumpfunktion auch die Einnahme von ACE-Hemmern, AT1-Rezeptorblockern und Aldosteronantagonisten beinhalten. Allerdings konnte nicht gezeigt werden, dass durch ACE-Hemmer und AT1-Rezeptorblocker die Mortalität gesenkt wird.

In der CHARM-Preserved- und der I-PRESERVE-Studie wurde der Einfluss der AT1Rezeptorblocker Candesartan und Irbesartan auf Morbidität und Mortalität bei Patienten mit chronischer Herzinsuffizienz und erhaltener linksventrikulärer EF untersucht. Candesartan 
(CHARM-Preserved) führt zu einer moderaten Reduktion der Hospitalisierungsrate und verbessert die NYHA- Klassifikation der herzinsuffizienten Patienten (Yusuf et al. 2003). Irbesartan (I-PRESERVE) hatte keinen Einfluss auf die Mortalität oder Hospitalisierung aufgrund kardiovaskulärer Ursachen bei Patienten mit Herzinsuffizienz und erhaltener systolischer LV-Funktion und führte zu keiner Verbesserung der sekundären Endpunkte oder krankheitsbezogener Lebensqualität (Massie et al. 2008).

Die PEP-CHF-Studie wiederum untersuchte die Wirkung des ACE-Hemmers Perindopril auf herzinsuffiziente Patienten mit erhaltener linksventrikulärer EF. Perindopril führte zu keinem statistisch signifikanten Vorteil beim primären Endpunkt aus Mortalität und ungeplanten Hospitalisierungen aufgrund der Herzinsuffizienz (Cleland et al. 1999). Für Aldosteronantagonisten fehlen größere Studien bisher vollkommen.

Betablocker und einige Calciumkanalblocker (Verapamil, Nifedipin, Diltiazem) können zur Prävention exzessiver Tachykardien eingesetzt werden. Dadurch soll die Herzfrequenz gesenkt, der Sinusrhythmus aufrecht erhalten und die Diastole verlängert werden. Die myokardiale Relaxation und die Ventrikelfüllung werden optimiert und eine Senkung des diastolischen Drucks und eine Verlängerung der koronaren Durchblutungszeit können somit erreicht werden (Zile und Brutsaert 2002b).

Der Gebrauch von ACE-Hemmern, Calciumkanalblockern und Betablockern reduziert Ischämien, optimiert die myokardiale Relaxation, limitiert die Hypertrophie und kann somit eine Verbesserung der diastolischen Funktion bewirken (Diller et al. 1999).

Ein weiterer Angriffspunkt ist die Verbesserung der Leistungsfähigkeit. Bei herzinsuffizienten Patienten mit erhaltener Ejektionsfraktion steigt unter Belastung die Herzfrequenz und eine Zunahme der Relaxationsgeschwindigkeit wird gleichzeitig verhindert. Der diastolische Druck steigt, das Schlagvolumen kann nicht ausreichend angehoben werden und die Patienten entwickeln Luftnot und Abgeschlagenheit. Betablocker, Calciumkanalblocker und AT1Rezeptorblocker verlangsamen die Herzfrequenz, verlängern die Diastole, bewirken eine günstige hämodynamische Anpassung und können somit günstige Effekte auf die Symptomatik und körperliche Belastbarkeit bei Patienten mit chronischer Herzinsuffizienz bei erhaltener Pumpfunktion ausüben (Zile und Brutsaert 2002 b). Ob eine Frequenzsenkung tatsächlich vorteilhaft ist, muss noch geprüft werden.

Positiv inotrope Substanzen wie Digitalis sind für Patienten mit isolierter chronischer Herzinsuffizienz und erhaltener Ejektionsfraktion nicht indiziert. In den Resultaten der Digitalis Investigation Group trial wurde aufgezeigt, dass herzinsuffiziente Patienten mit erhaltener Pumpfunktion weniger Symptome und eine geringere Hospitalisierungsrate unter Digitalistherapie aufweisen (Ahmed et al. 2008). Diese Effekte konnten allerdings nicht in 
anderen Studien bestätigt werden. Außerdem war kein Effekt auf die Mortalität nachweisbar (Zile und Brutsaert 2002 b).

Ebenso sollten die dem Krankheitsprozess zugrunde liegenden Mechanismen in Angriff genommen werden. Dies umfasst eine Verbesserung der Calciumhämostase, eine Reduktion der neurohumoralen Aktivität und die Prävention und Regression von Fibrose und Hypertrophie (Zile und Brutsaert 2002 b).

Kontrolliertes körperliches Training stellt eine weitere wesentliche Option zur Verbesserung der Lebensqualität und Leistungsfähigkeit bei HFPEF dar. Dies zeigten die Ergebnisse einer prospektiven, randomisierten, kontrollierten Pilotstudie: Die Ex-DHF-P-Studie untersuchte in einem Kollektiv aus chronisch herzinsuffizienten Patienten mit erhaltener Ejektionsfraktion den Effekt einer kontrollierten Trainingstherapie durch gezieltes Ausdauer- und Krafttraining im Vergleich zur Kontrollgruppe ohne Training über einen Zeitraum von drei Monaten. Als Ergebnis zeigte sich in der Trainingsgruppe eine Verbesserung verschiedener Parameter der Lebensqualität, der linksventrikulären diastolischen Funktion und der körperlichen Belastbarkeit sowie ein Anstieg des peak $\mathrm{VO}_{2}$ (Edelmann et al. 2011).

Damit gibt es im Gegensatz zur chronischen Herzinsuffizienz mit eingeschränkter Ejektionsfraktion für die mit erhaltener Pumpfunktion keine evidenzbasierten Behandlungsstrategien, welche eine Verbesserung der Morbidität, Mortalität und des klinischen Schweregrades belegen.

Es sind Behandlungsstrategien vonnöten, die die Symptomatik verbessern, die funktionelle Kapazität steigern, den Hospitalisierungsbedarf reduzieren und die Mortalität mindern. Nach der Symptomreduktion nimmt die Beseitigung der Grunderkrankung einen wichtigen Stellenwert ein. Während in einigen Studien bereits bewiesen wurde, dass das Vorhandensein von Komorbiditäten die Prognose bei herzinsuffizienten Patienten mit eingeschränkter Pumpfunktion herabsetzt, so ist der Einfluss dieser auf Herzinsuffizienz mit erhaltener Ejektionsfraktion nur wenig untersucht, weshalb deren Rolle als therapeutischer Angriffspunkt und besonders deren Stellenwert auf die Ausprägung der Symptomatik noch unklar ist.

Somit könnte die Diagnose und Behandlung vorhandener Komorbiditäten eine besonders bedeutende Therapieoption zur Reduktion der Symptomatik, Morbidität und ggf. Mortalität herzinsuffizienter Patienten auch mit normaler EF darstellen und könnte helfen, Patienten mit Herzinsuffizienzsymptomatik besser zu differenzieren. 


\subsection{Stellenwert der Komorbiditäten bei chronischer Herzinsuffizienz}

Komorbiditäten, wie Hypertonus, koronare Herzerkrankungen und Vitien spielen eine wichtige Rolle in der Entstehung einer chronischen Herzinsuffizienz und werden häufig erst mit der Manifestation einer Herzinsuffizienz diagnostiziert. Allein oder in Koexistenz mit vorhandenen Risikofaktoren beeinflussen sie ebenfalls die Ausprägung, den Progress und die Prognose einer bestehenden Herzinsuffizienz. Insbesondere nichtkardiale Komorbiditäten sind häufig bei herzinsuffizienten Patienten vertreten und zeigen einen Zusammenhang mit dem klinischen Bild der Erkrankung.

\subsubsection{Hypertension}

Die infolge eines Hypertonus entstehende Druckbelastung führt zu einer konzentrischen Hypertrophie. Diese hat einerseits einige koronare und cerebrovaskuläre Erkrankungen zur Folge und andererseits entsteht nach Überschreiten einer kritischen Grenze der Myokardhypertrophie eine relative Koronarinsuffizienz mit Abnahme der Leistungsfähigkeit, Apoptose, ventrikulärer Dilatation und diastolischer Dysfunktion und endet in einer Dekompensation der Herzinsuffizienz. Weiterhin bewirkt ein Hypertonus eine RAAS- und Sympathikusaktivierung mit gesteigerter Katecholaminausschüttung, was zu einer DownRegulation der Betarezeptoren, einer Erhöhung der Nachlast und somit zu einer weiteren Verschlechterung der Herzinsuffizienz führt.

In der Framingham-Kohorte von 5143 Personen ohne chronische Herzinsuffizienz, von denen 49\% eine Hypertonie aufwiesen, war das Risiko, innerhalb von 20 Jahren an einer chronischen Herzinsuffizienz (alle Formen, diagnostiziert nach den Framingham- Kriterien) zu erkranken, bei Männern mit Hypertonie um den Faktor 2 und bei Frauen mit Hypertonie um den Faktor 3 erhöht. Die Inzidenz stieg mit der Höhe des Blutdrucks (Levy et al. 1996).

Der Hypertonus ist somit ein Risikofaktor für kardiovaskuläre Erkrankungen, Morbidität sowie Mortalität und spielt eine Rolle in der Entstehung und Progression der Herzinsuffizienz. In einigen Studien wurde gezeigt, dass die Therapie der Hypertension das Risiko für Schlaganfall, systolische Herzinsuffizienz, Myokardinfarkt und Gesamtmortalität senkt. So wurden in der MERIT-HF-Studie die Effekte der Beta-1-Blockade versus Placebo auf Mortalität, Hospitalisierung, Symptomatik und Lebensqualität untersucht, mit dem Ergebnis, dass sich die Therapie mit Metoprolol auf Patienten mit chronischer systolischer Herzinsuffizienz und einer Hypertonievorgeschichte positiv auswirkt. Die Mortalität, die Hospitalisierung infolge fortschreitender Herzinsuffizienz und die Zahl der an plötzlichem Herztod Verstorbenen konnte signifikant gesenkt werden (Herlitz et al. 2002). 
Im Gegensatz dazu untersuchten Gustafsson et al. (2006) den Einfluss von Hypertension auf die Langzeitmortalität in einer Kohorte hospitalisierter Patienten mit kongestiver Herzinsuffizienz. Die Studie ergab, dass ein bestehender arterieller Hypertonus keinen Einfluss auf die Mortalität von Herzinsuffizienzpatienten hat (Gustafsson et al. 2006).

Eine hypertensive Erkrankung kann sich zu einer diastolischen Herzinsuffizienz fortentwickeln. Lam et al. (2007) fanden bei Hypertonikern mit HFNEF im Vergleich zu Hypertonikern mit einer asymptomatischen diastolischen Dysfunktion eine höhere linksventrikuläre Steifigkeit (höherer LVEDP und niedrigeres LVEDV). Die Effekte des Hypertonus auf die Prognose und Symptomatik herzinsuffizienter Patienten mit erhaltener Ejektionsfraktion wurden bisher nicht ausreichend analysiert.

\subsubsection{KHK}

Infolge eines Herzinfarktes kommt es zu einem strukturellen Remodeling mit kompensatorischer Hypertrophie, Narbenbildung, eingeschränkter Relaxation und restriktivem Füllmechanismus. Die zusätzliche Kollagenablagerung in Infarkt- und NichtInfarktarealen führt zu einer Progression der LV-Dilatation, Myokardsteifigkeit, diastolischer und/oder systolischer Dysfunktion mit der Folge von Herzinsuffizienz und Herztod nach abgelaufenem Myokardinfarkt.

Die koronare Herzerkrankung ist somit neben dem Hypertonus ein bedeutender Risikofaktor für die Entstehung und auch Progression der Herzinsuffizienz. Insbesondere rezidivierende Myokardinfarkte, die in seltenen Fällen bei herzinsuffizienten Patienten erkannt werden, sind eine wichtige Ursache für einen plötzlichen Herztod und die Progression der Herzinsuffizienz.

Daher sollte bei Patienten mit Herzinsuffizienz zur Prävention der Herzinsuffizienzprogression ein Hauptaugenmerk auf die Behandlung und Vermeidung von Ischämie und koronaren Ereignissen gelegt werden (Cleland et al. 2003).

Welchen Einfluss eine KHK auf die Symptomatik und die Prognose herzinsuffizienter Patienten mit erhaltener Pumpfunktion hat, ist dabei noch unklar.

\subsubsection{Hyperurikämie}

Hyperurikämie entsteht häufig bei Patienten mit Herzinsuffizienz infolge einer Schleifendiuretikatherapie oder durch renale Dysfunktion.

Hohe Harnsäurespiegel (>8,5 mg/dl) führen zu einem 8-fachem Risiko einer Niereninsuffizienz (Tomita et al. 2000). Ferner haben Klein et al. (1973) gezeigt, dass ein linearer Zusammenhang zwischen Serumharnsäurespiegel und Blutdruck mit der Folge eines Hypertonus bei hohem Harnsäurelevel besteht. Ebenso konnte in älteren 
epidemiologischen Studien gezeigt werden, dass Hyperurikämie einen Risikofaktor für kardiovaskuläre Erkrankungen darstellt. Sie ist ein Zeichen für eine metabolische Imbalance und zudem ein unabhängiger Marker für eine ungünstige Prognose bei Patienten mit chronischer Herzinsuffizienz (Edwards 2008).

Allerdings ist der direkte Einfluss der Hyperurikämie auf den Verlauf und die Ausprägung der chronischen Herzinsuffizienz bei erhaltener sowie eingeschränkter Ejektionsfraktion nicht systematisch analysiert und somit ist der Effekt auf die Symptomatik der Patienten noch unbekannt.

\subsubsection{Diabetes mellitus}

Diabetes mellitus ist eine häufige Komorbidität bei Herzinsuffizienz und betrifft 20 bis 30\% der Patienten. Diabetes verursacht funktionelle, morphologische und biochemische Veränderungen am Myokard, die unabhängig von anderen Faktoren zu einer LVHypertrophie, diastolischer Dysfunktion und Herzinsuffizienz führen können. Analysen der Framinghampopulation zeigen eine 2-fach höhere Inzidenz der Herzinsuffizienz bei Männern mit Diabetes und eine 5-fach höhere bei Frauen mit Diabetes (Resl et al. 2009). Ebenfalls ist die Hospitalisierungsrate wegen Herzinsuffizienz bei Patienten mit Diabetes mellitus höher. Diabetiker mit Herzinsuffizienz leiden an stärker ausgeprägten Herzinsuffizienzsymptomen, reduzierter Leistungsfähigkeit, eingeschränkter Lungenfunktion und einem schnelleren Progress der Symptomatik (MacDonald et al. 2008).

Diabetes mellitus, HbA1c und ein erhöhter BMI sind Risikofaktoren für die Entstehung kardiovaskulärer Erkrankungen und Herzinsuffizienz. Ein direkter Zusammenhang konnte bei Iribarren et al. gezeigt werden: Ein $\mathrm{HbA} 1 \mathrm{c} \geq 10$ verglichen mit einem $\mathrm{HbA} 1 \mathrm{c} \leq 7$ ist mit einem 1,56-fachen Risikoanstieg für Herzinsuffizienz verbunden. Zudem ist der Anstieg des HbA1c um 1\% mit einem um 8\% erhöhten Risiko, an einer Herzinsuffizienz zu erkranken, assoziiert (Iribarren et al. 2001).

\subsubsection{Adipositas}

Adipositas bewirkt die Entstehung eines Hypertonus und umgekehrt kann ein Hypertonus die Entstehung einer Adipositas begünstigen. Während Adipositas eine Kammerdilatation ohne Wanddickenzunahme zur Folge hat, entsteht aufgrund der Hypertonie eine konzentrische linksventrikuläre Hypertrophie ohne Kammerdilatation. Da aber Übergewicht und Hypertonie häufig koexistieren, entstehen infolge dessen linksventrikuläre systolische sowie diastolische Dysfunktionen.

Adipositas ist assoziiert mit einem hohen Risiko für Morbidität und Mortalität in der gesamten Population. Sie führt zu einer schnelleren Progression der kardiovaskulären Risikofaktoren, 
was wiederum das Risiko für Herzinsuffizienz und koronarer Herzerkrankung erhöht (Artham et al. 2008).

Für jede Zunahme des BMI um $1 \mathrm{~kg} / \mathrm{m}^{2}$ steigt das Herzinsuffizienzrisiko um $5 \%$ bei Männern und um $7 \%$ bei Frauen. Bei Übergewicht besteht eine höhere Prävalenz für LVAbnormalitäten, was mit einer höheren Mortalität assoziiert ist. Aber ein höherer BMI geht einher mit einer geringeren Mortalität (obesity paradox) (Lavie et al. 2009).

So haben Gustafsson et al. den Einfluss des BMIs auf die Herzinsuffizienz-Mortalität in 4700 akut hospitalisierten Patienten untersucht: Bei nicht-systolischer Herzinsuffizienz waren die Überlebensraten bei übergewichtigen Patienten im Vergleich zu Untergewichtigen höher. Bei Patienten mit systolischer Herzinsuffizienz folgte die Überlebensrate einer U-Kurve (Artham et al. 2008).

In der DIG-Studie (7788 Patienten mit stabiler Herzinsuffizienz und Sinusrhythmus; Digoxin versus Placebo) konnte gezeigt werden, dass ein Zusammenhang zwischen einem höheren BMI und niedrigerer Mortalität bei Patienten mit Herzinsuffizienz besteht. Übergewichtige Patienten hatten weniger schwere myokardiale Dysfunktionen als normgewichtige. Patienten mit stabiler Herzinsuffizienz und hohem BMI haben ein geringeres Gesamtmortalitätsrisiko und ein niedrigeres Risiko, an fortschreitender Herzinsuffizienz zu versterben. Ebenfalls konnten im Vergleich zu Normgewichtigen bessere Überlebensraten und bessere Endergebnisse nachgewiesen werden (Curtis et al. 2005).

\subsubsection{Hyperlipidämie}

Es ist bereits bewiesen, dass eine Adipositas mit einem hohen Risiko für Morbidität und Mortalität in der gesamten Bevölkerung einhergeht (Artham et al. 2008). Allerdings konnte der Effekt einer vorhandenen Hyperlipidämie auf die Prognose, die Mortalität und die Ausprägung der Symptomatik bei herzinsuffizienten Patienten mit erhaltener sowie reduzierter Ejektionsfraktion noch nicht nachgewiesen werden.

\subsubsection{Anämie}

Die Prävalenz der Anämie beträgt bei der chronischen Herzinsuffizienz 20 bis $50 \%$ und steigt mit zunehmender Schwere der Herzinsuffizienz, fortschreitendem Alter, renaler Dysfunktion und anderen vorhandenen Komorbiditäten (Angermann 2009).

Die Anämie führt bei Herzinsuffizienzpatienten zu linksventrikulärer Hypertrophie und einer schwereren diastolischen sowie systolischen Dysfunktion mit höherer NYHA-Klassifizierung, niedrigerer Leistungsfähigkeit, schlechterer Lebensqualität, einem Abfall der Nierenfunktion, stärker ausgeprägten peripheren Ödemen, niedrigerem Blutdruck, höherem Gebrauch an 
Diuretika und anderen kardiovaskulären Medikamenten und schlechteren neurohormonellen Profilen und inflammatorischen Markern (Zytokine, CRP) (Anand 2008).

In kontrollierten und nicht-kontrollierten Studien führte die Korrektur der Anämie (mit EPOstimulierenden Agenzien in Verbindung mit oraler- oder i.v.- Eisenzufuhr) zur Besserung des klinischen Status, zu verminderter Hospitalisierungsrate und zur Besserung der kardialen und renalen Funktion (Angermann 2009).

Eine vorhandene Anämie geht einher mit einer reduzierten Überlebensrate, steigender Morbidität und einer höheren Hospitalisierungsrate (Angermann 2009). So haben Horwich et al. einen Anstieg der Mortalität um 13\% für einen Abfall des Hämoglobins um $1 \mathrm{~g} / \mathrm{dl}$ nachgewiesen (Horwich et al. 2002).

\subsubsection{Renale Dysfunktion}

Die Prävalenz der renalen Dysfunktion nimmt mit steigender Schwere der Herzinsuffizienz, fortgeschrittenem Alter und dem Vorhandensein von Diabetes mellitus und Hypertonie zu.

Eine reduzierte glomeruläre Filtrationsrate fördert Hypertonie und Dyslipidämie und ist ebenfalls assoziiert mit LV-Hypertrophie, Inflammation, endothelialer Dysfunktion, Hyperkoagulabilität, Atherosklerose, Anämie und Urämie und entspricht damit einem potentiellen kardiovaskulärem Risikofaktor (Angermann 2009).

Hillege et al. (2000) haben bewiesen, dass sich die GFR unabhängig von der linksventrikulären Ejektionsfraktion verhält und bezüglich stabiler Herzinsuffizienz ein stärkerer Prädiktor der Mortalität ist, verglichen mit der NYHA- Klassifizierung und der LVEF.

Philipp et al. haben die Korrelation zwischen Nierenfunktion und Symptomatik untersucht: Mit sinkender GFR steigt die NYHA-Klassifizierung (NYHA I bei einer GFR von $82 \mathrm{ml} / \mathrm{min}$, NYHA IV bei einer GFR von $59 \mathrm{ml} / \mathrm{min}$ ) (Besarab und Soman 2005, S. 366).

Eine eingeschränkte GFR ist dementsprechend mit einer Progression kardiovaskulärer Erkrankungen assoziiert und führt zu steigender Gesamtmortalität und kardiovaskulärer Mortalität. Ebenfalls führt eine renale Dysfunktion zu einem schlechterem „Outcome“ und steigender Mortalität bei Herzinsuffizienzpatienten (Ford et al. 2009).

\subsubsection{COPD}

Die Prävalenz der COPD beträgt bei Patienten mit chronischer Herzinsuffizienz 20 bis 30\%. Eine chronische Herzinsuffizienz bei vorherrschender COPD aufzudecken, ist eine Herausforderung, da sich typische Symptome und Zeichen wie Dyspnoe eindeutig überschneiden. Die Erhebung des natriuretischen Peptids (BNP oder NT-proBNP) oder die Durchführung einer Echokardiographie kann für die Diagnosestellung hilfreich sein. 
Der Zigarettenkonsum - die häufigste Ursache der COPD - führt neben der Entstehung von Entzündung, Obstruktion und Emphysemen in den Atemwegen, zu systemischen Entzündungen, vasomotorischer und endothelialer Dysfunktion, einem Anstieg prokoagulatorischer und inflammatorischer Faktoren und damit zu einem dramatischen Anstieg des kardiovaskulären Risikos bei Patienten mit COPD. Diese Patienten weisen ein erhöhtes Risiko für die Entstehung einer Herzinsuffizienz auf. COPD ist ein sicherer unabhängiger Risikofaktor für kardiovaskuläre Erkrankungen, einschließlich Herzinsuffizienz, und für kardiovaskuläre Mortalität. Vor allem wirkt sich eine Koexistenz von COPD und chronischer Herzinsuffizienz negativ auf den prognostischen Verlauf und die Leistungsfähigkeit aus und geht mit Entzündungen und Skelettmuskeldysfunktionen einher (Angermann 2009).

Boudestein et al. (2009) konnten zeigen, dass Herzinsuffizienz ein sicherer unabhängiger Prädiktor für die Gesamtmortalität bei Patienten mit COPD ist. Neben der signifikant höheren Mortalität ist ebenfalls die Hospitalisierungsrate bei COPD-Patienten mit gleichzeitig bestehender Herzinsuffizienz höher, verglichen mit COPD-Patienten ohne Herzinsuffizienz (Boudestein et al. 2009).

\subsection{Fragestellung und Zielsetzung der vorliegenden Arbeit}

Ziel dieser Arbeit ist es, den genauen Zusammenhang ausgewählter typischer Komorbiditäten (Diabetes mellitus, Hyperurikämie, renale Dysfunktion, Anämie, COPD, Koronare Herzerkrankung, Hypertonus, Adipositas, Hyperlipidämie) mit der chronischen Herzinsuffizienz zu analysieren.

Komorbiditäten tragen in hohem Maß zur Morbidität und Mortalität bei chronischer Herzinsuffizienz mit reduzierter Ejektionsfraktion bei. Allerdings ist deren Auswirkung auf die Ausprägung der Symptomatik bei chronisch herzinsuffizienten Patienten mit erhaltener Pumpfunktion nicht systematisch analysiert worden.

Zum einen soll in dieser Arbeit die Verteilung von Komorbiditäten in einem großen Herzinsuffizienzkollektiv untersucht und diese im Vergleich bei reduzierter sowie erhaltener Pumpfunktion betrachtet werden. Zum anderen spielt die Erfassung der Symptome und deren Verteilung in diesem Kollektiv eine Rolle. Des Weiteren soll der Einfluss der Komorbiditäten auf die Ausprägung der Symptomatik herzinsuffizienter Patienten erarbeitet werden.

Die Ergebnisse sollen helfen, die Diagnosestellung der chronischen Herzinsuffizienz, besonders der mit erhaltener Ejektionsfraktion zu verbessern. Ebenso sollen mit dem damit verbundenen pathologischen Verständnis Therapieoptionen für die HFPEF erarbeitet werden. 


\section{Material und Methoden}

\subsection{Patienten}

Das untersuchte Kollektiv umfasst Patienten mit Herzinsuffizienz, die in dem Zeitraum $11 / 2003$ bis $12 / 2008$ prospektiv in das deutsche multizentrische Kompetenznetz Herzinsuffizienz (KNHI) aufgenommen worden sind (http://www.knhi.de). Insgesamt elf Studien, die man im Rahmen von Teilprojekten des KNHI durchgeführt hat, werden in die vorliegende Analyse einbezogen. Diese Teilprojekte beschäftigen sich mit unterschiedlichen Schwerpunkten: mit Ätiologie, Epidemiologie, Diagnostik, Verlauf, Management und Therapie der chronischen Herzinsuffizienz. Auch die Rolle von Risikofaktoren und der Einfluss auf die Lebensqualität sind im Rahmen einiger Teilprojekte untersucht worden. Eine Zusammenfassung über die individuellen Ein- und Ausschlusskriterien befindet sich im Anhang (6.2).

\subsection{Teilprojekte}

- Gen-Studie (TP5)

Ätiologie der Herzinsuffizienz im Kompetenznetz Herzinsuffizienz

- Handheld-BNP (TP6)

Objektivierung der kardiovaskulären Dysfunktion im ambulanten und hausärztlichen Bereich mittels handgehaltener Echokardiographie und dem BNP-Schnelltest

- $\quad$ Diast-CHF (TP7)

Prävalenz und Verlauf der diastolischen Dysfunktion und der diastolischen Herzinsuffizienz

- Ex-DHF-P (TP7)

Exercise training in diastolic heart failure-pilotstudy

- IKARIUS (TP9A)

Die inflammatorische dilatative Kardiomyopathie als Manifestation einer autoimmunen Diathese

- HIV \& Herz (TP9B)

Chronische Herzinsuffizienz bei HIV-positiven Patienten: Studie zu kardialen Erkrankungen bei von der HIV-Infektion betroffene Patienten

- $\quad$ HELPS (TP10)

Heidelberger Studie zur Verlaufsuntersuchung von Lebensqualität und psychischer Komorbidität bei depressiven Herzinsuffizienz-Patienten

- $\quad$ TtT (TP10) 
Schulung von Hausärzten zur Verbesserung der Lebensqualität von Patienten chronischer Herzinsuffizienz

- CIBIS-ELD (TP12)

Vergleich von Bisoprolol mit Carvedilol bei älteren Patienten mit Herzinsuffizienz

- $\quad$ ACVB-Out (TP13)

Geschlechtsspezifische Unterschiede in der Bedeutung von Herzinsuffizienz, Hormonstatus und psychosozialen Faktoren für die Prognose nach aortokoronarer Bypass-Operation

- $\quad$ INH (TP15)

Krankheitsmanifestation und Krankheitsmanagement bei chronischer Herzinsuffizienz

\subsection{Untersuchungen}

\subsubsection{Anamnese}

Im Rahmen der Teilprojekte ist standardisiert ein ausführlicher Basisdatensatz erhoben worden.

Die Anamnese umfasst die Krankengeschichte und die aktuelle Symptomatik der Patienten sowie die Erhebung soziodemographischer Angaben. Ebenfalls sind die Patienten nach der Anzahl der Kontakte zum Hausarzt und Kardiologen und nach stationären Rehabilitations-I Kur- und Krankenhausaufenthalten innerhalb der letzten 12 Monate befragt worden.

Anschließend ist das Vorhandensein kardiovaskulärer Risikofaktoren geprüft worden. Dabei handelt es sich um Diabetes mellitus, Hypertonie (bei aktuellem Blutdruck $>140 / 90 \mathrm{mmHg}$, bei bestehender Therapie mit Antihypertensiva oder bekannt als Vordiagnose), Hyperlipidämie (bei bekannter Vordiagnose oder bei bestehender Therapie mit Lipidsenkern), Hyperurikämie (bei Serum- Harnsäure $>6,4 \mathrm{mg} / \mathrm{dl}$ oder bestehender Therapie mit Harnsäuresenkern), Myokardinfarkt vor dem 60. Lebensjahr bei leiblichen Eltern, Geschwistern oder Kindern, Raucherstatus und Alkoholkonsum.

Bezüglich der Krankengeschichte sind aus der Anamnese und aus Vorbefunden kardiale Diagnosen erhoben worden. Diese sind im Detail das Bestehen einer koronaren Herzkrankheit, der Zustand nach Myokardinfarkt, primäre Herzklappenerkrankungen, angeborene Herzfehler, eine vorliegende Kardiomyopathie und deren Ätiologie. Zudem sind bisherige kardiovaskuläre Interventionen, wie koronare Revaskularisation, Bypass- oder Herzklappen-Operationen, Schrittmacher- und Defibrillatorimplantationen, Herztransplantationen und Reanimationsmaßnahmen, erfasst worden.

Außerdem wurden die Patienten bezüglich bekannter aktueller Nebendiagnosen befragt. Diese beinhalten PAVK, cerebrovaskuläre Erkrankungen, Synkopen in der Vorgeschichte, COPD, pulmonale Hypertonie, Depression (entweder bekannt als Vordiagnose oder aktuelle 
Einnahme antidepressiver Medikamente), HIV-Infektionen, sowie chronische Hepatitis A oder C, Leberzirrhose und maligne Erkrankungen. Frauen wurden zusätzlich nach dem Zeitpunkt der Menopause befragt.

Bei der aktuellen regelmäßigen Medikation ist die Einnahme kardiovaskulärer Medikamente, Lipidsenker, Antikoagulanzien, Antidiabetika, Antidepressiva, Schlaf-/ Beruhigungsmittel und Allopurinol erhoben worden. Bei Frauen hat man ergänzend die Einnahme oraler Kontrazeptiva und postmenopausaler Hormonen ermittelt.

\subsubsection{Körperliche Untersuchung}

Im Rahmen einer klinischen Untersuchung hat man Größe, Gewicht, Blutdruck und Herzfrequenz bestimmt und Symptome und Zeichen der Herzinsuffizienz, wie das Vorhandensein von Belastungs- und/oder Ruhedyspnoe, erfasst. Eine orientierende abdominelle Untersuchung hat der Prüfung von Aszites, Hepatomegalie und Halsvenenstauung gedient. Ebenfalls ist eine Untersuchung auf das Vorliegen von peripheren Ödemen und pulmonalen Rasselgeräuschen erfolgt. Auch der Zeitpunkt der Erstdiagnose der Herzinsuffizienz wurde ermittelt. Neben der Klassifikation der Herzinsuffizienz nach der Lokalisation, erfolgte anhand der Leistungsfähigkeit und der Schwere der Symptomatik der Patienten, die Einteilung der vorliegenden Herzinsuffizienz nach der New-York-Heart-Association (NYHA)- Klassifikation.

Außerdem hat der Untersucher mit Hilfe der Major- und Minor- Kriterien der FraminghamDefinition (Ho et al. 1993) das Vorliegen einer symptomatischen Herzinsuffizienz beurteilt. Die Major-Kriterien beinhalten paroxysmale nächtliche Dyspnoe, Halsvenenstauung, pulmonale Rasselgeräusche, den radiologische Nachweis einer Kardiomegalie, ein akutes Lungenödem, einen dritten Herzton und hepatojugulären Reflux. Zu den Minor-Kriterien gehören nächtlicher Husten, beidseitige Knöchelödeme, Luftnot bei normaler Belastung, Hepatomegalie, ein Pleuraerguss sowie eine Tachykardie (>120 Schläge/min). Die Diagnose einer Herzinsuffizienz wurde gestellt, wenn zwei Hauptkriterien oder ein Haupt- und zwei Nebenkriterien vorhanden waren.

\subsubsection{Basislabordiagnostik}

Bei jedem Patienten ist ein Basislabor erhoben worden, welches bei klinisch stabilen Patienten maximal drei Monate alt sein durfte und sonst aktuell durchgeführt werden musste. Im Detail sind dabei Hämoglobin, Kreatinin, Harnsäure, Natrium, Kalium und Gesamtcholesterin bestimmt worden. Die ermittelten Ergebnisse sind daraufhin in die Diagnose der Risikofaktoren und Komorbiditäten eingeflossen. 


\subsubsection{Elektrokardiogramm}

Die Auswertung eines EKGs erfolgte bei jedem Patienten. Bei klinisch stabilen Patienten durfte das EKG nicht älter als drei Monate sein und musste sonst aktuell durchgeführt werden. Zur Auswertung wurden Herzfrequenz, Rhythmus (Sinusrhythmus, Vorhofflimmern oder ein anderer Rhythmus), PQ- Zeit, QRS- Dauer und QT- Zeit bestimmt. Ebenfalls wurde das EKG auf Vorliegen eines Schrittmacherrhythmus, AV-Block, Links- oder Rechtsschenkelblock geprüft.

\subsubsection{Echokardiographie}

Bei allen Patienten wurde eine aktuelle, transthorakale, zweidimensionale Echokardiographie durchgeführt. Die Durchführung erfolgte gemäß der Richtlinien des Basisdatensatzes des KNHI (SOP KNHI). Diese beinhalten die Erfassung der Dimensionen (LVDES, LVDED, IVSED, PWED, LAES), die linksventrikuläre Ejektionsfraktion (LVEF), das Vorhandensein diverser Herzklappenfehler, einer RV Dilatation oder eines Perikardergusses.

Anhand der linksventrikulären Ejektionsfraktion, bestimmt nach Simpson (XX), wurden die symptomatischen Patienten in die Gruppen der Herzinsuffizienz mit eingeschränkter Pumpfunktion (LVEF<50\%) und erhaltener Pumpfunktion (LVEF $\geq 50 \%$ ) eingeteilt.

\subsubsection{Herzkatheteruntersuchung}

War bei den Patienten bereits eine Herzkatheteruntersuchung durchgeführt worden, so wurden die Ergebnisse der letzten HKU dokumentiert. Aufgenommen wurde, ob und in welchem Ausmaß eine koronare Herzkrankheit vorlag, ob eine Biopsie entnommen wurde und ob eine Rechtsherzkatheteruntersuchung erfolgt war.

\subsection{Statistik}

Die statistischen Analysen erfolgten mit dem Programm SPSS (IBM Inc.), Version 19.

Alle Werte sind als Mittelwert $( \pm S D$ ) oder prozentualer Anteil (\%) aufgeführt. Vergleiche zwischen den Gruppen wurden mittels Student-t-test oder bei dichotomer Verteilung mit Fishers Exact test durchgeführt.

Der Einfluss der unterschiedlichen Komorbiditäten auf die Ausprägung der Symptomatik anhand der NYHA-Klasse wurde mittels ordinaler logistischer Regression mit der SPSSProzedur PLUM (Polytomous Universal Model) überprüft, adjustiert für Alter, Geschlecht und linksventrikuläre Ejektionsfraktion bzw. Herzinsuffizienzgruppe (HFPEF bzw. HFREF) und 
wurde als Odds Ratio (OR, 95\% Konfidenzintervall) angegeben. Die NYHA-Klasse war die abhängige Variable und gleichzeitig wurden alle Regressoren eingeschlossen. Allgemein ist eine ordinale Regressionsanalyse dafür konzipiert, die Zusammanhänge zwischen einer ordinalen abhängigen Variablen (deren Werte zwar in eine Reihenfolge zu ordnen sind, deren Abstände zwischen den Werten jedoch nicht quantifizierbar sind) und kategorialen und/oder intervallskalierten erklärenden Variablen zu untersuchen.

Im Anhang ist exemplarisch für die Komorbidität Diabetes ein entsprechendes SPSSOutputdokument dargestellt.

Des Weiteren erfolgten multivariate Regressionsanalysen für die NYHA-Klassen. Dabei wurden alle Komorbiditäten als Kovariaten behandelt. Bei der multivariaten Regressionsanalyse handelt es sich um eine multiple ordinale logistische Regressionsrechnung aller Komorbiditäten, die einzeln einen signifikanten Einfluss auf die NYHA-Klasse aufweisen, anhand derer die Beständigkeit des Zusammenhangs, auch in Anwesenheit anderer Faktoren überprüft werden soll. Daher wurden ergänzend noch zwei echokardiographische Parameter in die Analyse aufgenommen: die linksventrikuläre Ejektionsfraktion zur Abschätzung der Funktionseinschränkung bei HFREF sowie der linksventrikuläre enddiastolische Diameter (LVDED) zur Abschätzung der Füllungseinschränkung bei HFPEF.

Im Anhang ist exemplarisch ein SPSS-Outputdokument der multivariaten Analyse aufgeführt, jedoch ohne Hinzunahme der echokardiographischen Parameter. 


\section{Ergebnisse}

\subsection{Deskriptive Statistik des Patientenkollektivs}

\subsubsection{Patienten und wissenschaftliche Projekte}

In den Tabellen dieses Abschnitts, ist die Zusammensetzung des Patientenkollektivs aufgeführt. In dem Zeitraum 2003 bis 2008 sind insgesamt 8368 Patienten im Rahmen von Studien des deutschen Kompetenznetzes Herzinsuffizienz (KNHI) aufgenommen und über einen bestimmten Zeitraum untersucht worden. Durch dieses Screening wurde bei 4259 Patienten eine Herzinsuffizienz diagnostiziert, während die restlichen 4109 Patienten keine Herzinsuffizienz aufwiesen. Davon konnten schließlich 4079 Patienten mit Herzinsuffizienz für die Analysen gewonnen werden, wovon 2674 (65,6\%) Männer und 1405 (34,4\%) Frauen sind. Die genaue Zusammenstellung des Kollektivs ist in Abbildung 1 dargestellt.

Insgesamt sind Daten aus elf Studien des $\mathrm{KNHI}$ in die Analyse einbezogen worden. Diese wissenschaftlichen Projekte und deren Anteil am gesamten Patientenkollektiv sind in Tab.1 aufgelistet.

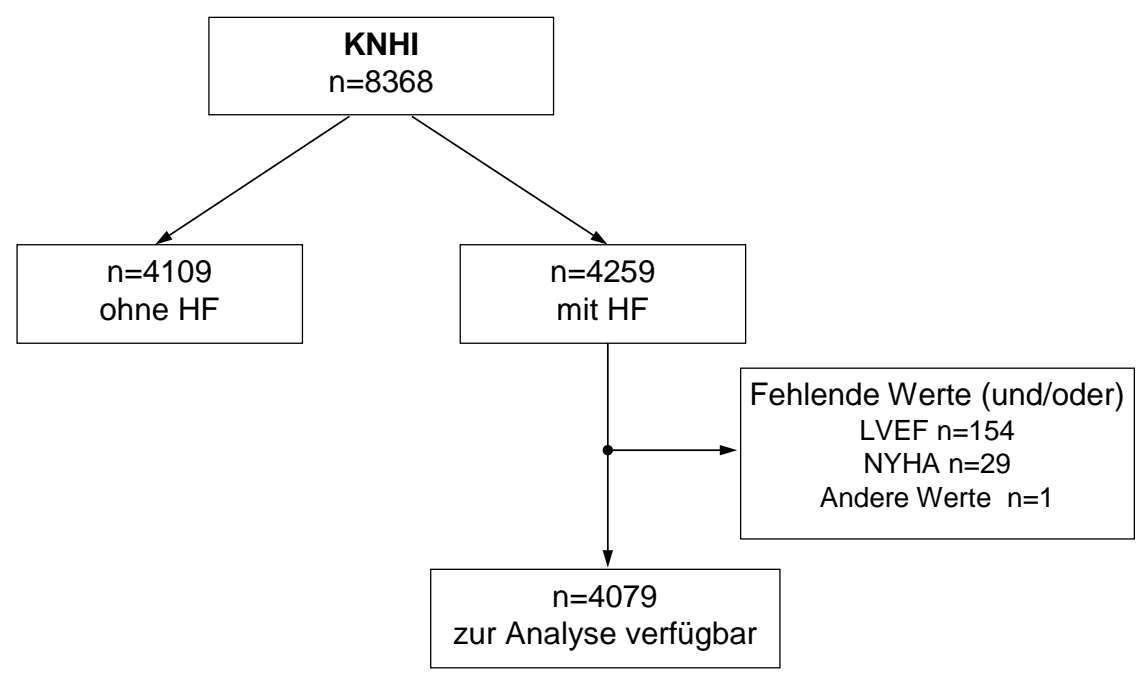

Abbildung 1: Dargestellt ist die Zusammensetzung des Patientenkollektivs, im Rahmen des deutschen Kompetenznetz Herzinsuffizienz. 


\begin{tabular}{|c|c|c|c|c|c|}
\hline & $\begin{array}{c}\text { LVEF }<50 \% \\
n\end{array}$ & $\begin{array}{l}\text { LVEF }<50 \% \\
\text { Prozent }[\%]\end{array}$ & $\begin{array}{c}\text { LVEF } \geq 50 \% \\
\mathrm{n}\end{array}$ & $\begin{array}{l}\text { LVEF } \geq 50 \% \\
\text { Prozent [\%] }\end{array}$ & $\begin{array}{c}\text { Patienten } \\
\text { Gesamt }\end{array}$ \\
\hline $\mathrm{HH}-\mathrm{BNP}$ & 50 & 12 & 355 & 88 & 40 \\
\hline $\mathrm{INH}$ & 877 & 99 & 7 & 1 & 884 \\
\hline Diast-CHF & 59 & 24 & 191 & 76 & 250 \\
\hline HIV\&Herz & 21 & 36 & 38 & 64 & 59 \\
\hline Gen & 519 & 71 & 217 & 29 & 736 \\
\hline $\begin{array}{l}\text { CIBIS- } \\
\text { ELD }\end{array}$ & 219 & 50 & 218 & 50 & 437 \\
\hline Ikarius & 331 & 99 & 3 & 1 & 334 \\
\hline HELPS & 295 & 92 & 27 & 8 & 322 \\
\hline $\mathrm{TtT}$ & 151 & 91 & 15 & 9 & 166 \\
\hline ACVB-Out & 263 & 67 & 132 & 33 & 395 \\
\hline \multirow[t]{2}{*}{ Ex-DHF-P } & 0 & 0 & 91 & 100 & 91 \\
\hline & 2785 & 68 & 1294 & 32 & 4079 \\
\hline
\end{tabular}

Tabelle 1: Aufgeführt sind die Teilprojekte des KNHIs, die in die Analyse einbezogen worden sind, und deren Anteil der Patienten am Gesamtkollektiv.

\subsubsection{Grundlegende Patientendaten}

Die folgende Tabelle zeigt die Verteilung demographischer Variablen wie Alter und Geschlecht sowie die Verteilung der zum Ausgangszeitpunkt gemessenen klinischen Charakteristika wie Blutdruck, Herzfrequenz und die Häufigkeit der Medikation mit kardiovaskulärer Indikation im gesamten Patientenkollektiv.

Ein Vergleich der Verteilung erfolgt zwischen den Gruppen der Herzinsuffizienz mit eingeschränkter Pumpfunktion (LVEF<50\%) und erhaltener Pumpfunktion (LVEF $\geq 50 \%$ ). Alle Werte sind als Mittelwert ( \pm SD) oder prozentualer Anteil (\%) aufgeführt. Der p-Wert stellt die Signifikanzen für die Unterschiede in der Gruppenverteilung dar.

Herzinsuffiziente Patienten mit erhaltener Ejektionsfraktion sind im Vergleich zu Patienten mit eingeschränkter EF durch ein höheres Alter und meist weibliches Geschlecht gekennzeichnet. Darüber hinaus zeichnen sie sich durch einen höheren Blutdruck, eine niedrigere Herzfrequenz in Ruhe und eine weniger häufige Einnahme kardiovaskulärer Medikamente aus. 


\begin{tabular}{|c|c|c|c|c|c|}
\hline & $\begin{array}{c}\text { LVEF<50 } \\
\% \\
n=2785\end{array}$ & $\begin{array}{c}\text { LVEF }<50 \\
\% \\
\text { Prozent } \\
{[\%]}\end{array}$ & $\begin{array}{c}\text { LVEF } \geq 50 \\
\% \\
n=1294\end{array}$ & $\begin{array}{c}\text { LVEF } \geq 50 \\
\% \\
\text { Prozent } \\
{[\%]}\end{array}$ & $\mathrm{p}$-Wert \\
\hline Alter [Jahre] & $63 \pm 14$ & & $67 \pm 13$ & & $<0,001$ \\
\hline $\begin{array}{l}\text { Weibliches } \\
\text { Geschlecht [n] }\end{array}$ & 709 & 25,5 & 696 & 53,8 & $<0,001$ \\
\hline $\begin{array}{l}\text { Systolischer } \\
\text { Blutdruck } \\
\text { [mmHg] }\end{array}$ & $123 \pm 20$ & & $142 \pm 24$ & & $<0,001$ \\
\hline $\begin{array}{l}\text { Diastolischer } \\
\text { Blutdruck } \\
{[\mathrm{mmHg}]}\end{array}$ & $73 \pm 12$ & & $81 \pm 13$ & & $<0,001$ \\
\hline $\begin{array}{l}\text { Herzfrequenz } \\
\text { [1/min] }\end{array}$ & $76 \pm 17$ & & $70 \pm 14$ & & $<0,001$ \\
\hline ACE-I/AT1-RA [n] & 2458 & 88,3 & 790 & 61,1 & $<0,001$ \\
\hline Betablocker [n] & 2352 & 84,5 & 669 & 51,7 & $<0,001$ \\
\hline Diuretika [n] & 2213 & 79,5 & 680 & 52,6 & $<0,001$ \\
\hline $\begin{array}{l}\text { Aldosteron- } \\
\text { Antagonisten[n] }\end{array}$ & 1290 & 46,3 & 61 & 4,7 & $<0,001$ \\
\hline
\end{tabular}

Tabelle 2: Die Tabelle zeigt demographische Variablen und klinische Charakteristika des Patientenkollektivs im Vergleich von eingeschränkter und erhaltener Ejektionsfraktion.

\subsubsection{Zeichen und Symptome der Herzinsuffizienz im Patientenkollektiv}

In diesem Abschnitt finden sich Tabellen und Abbildungen, die die Symptomatik der Herzinsuffizienz und ihre Häufigkeitsverteilung im Kollektiv verdeutlichen.

Die Tabelle 3 zeigt eine Auswahl durch Anamnese und körperliche Untersuchung erhobener Symptome der Herzinsuffizienz und deren Verteilung im Vergleich zwischen eingeschränkter und erhaltener Ejektionsfraktion.

Ebenfalls aufgeführt sind die Verteilung der NYHA-Klasse und die Anzahl der Symptome. Alle Werte sind als Mittelwert ( $\pm \mathrm{SD}$ ) oder prozentualer Anteil aufgeführt (\%). Der p-Wert stellt die Signifikanz für die Unterschiede in der Gruppenverteilung dar. 
Patienten mit erhaltener Ejektionsfraktion weisen, abgesehen von dem Vorhandensein peripherer Ödeme, insgesamt weniger Symptome auf, als Patienten mit eingeschränkter Pumpfunktion.

\begin{tabular}{|l|c|c|c|c|c|}
\hline & $\begin{array}{c}\text { LVEF }<50 \\
\% \\
\mathrm{n}=2785\end{array}$ & $\begin{array}{c}\text { LVEF }<50 \\
\% \\
\text { Prozent } \\
{[\%]}\end{array}$ & $\begin{array}{c}\text { LVEF } \geq 50 \\
\% \\
\mathrm{n}=1294\end{array}$ & $\begin{array}{c}\text { LVEF } \geq 50 \\
\% \\
\text { Prozent } \\
{[\%]}\end{array}$ & p-Wert \\
\hline Belastungsdyspnoe & 2424 & 87,0 & 1088 & 84,1 & $<0,05$ \\
Ruhedyspnoe & 327 & 11,7 & 88 & 6,8 & $<0,001$ \\
Periphere Ödeme & 713 & 25,6 & 432 & 33,4 & $<0,001$ \\
Halsvenenstauung & 187 & 6,7 & 31 & 2,4 & $<0,001$ \\
Pulmonale & 317 & 11,4 & 52 & 4,0 & $<0,001$ \\
Rasselgeräusche & $2,4 \pm 0,6$ & & $2,0 \pm 0,6$ & & \\
\hline NYHA- Klasse & $1,4 \pm 1,0$ & & $1,3 \pm 0,8$ & & $<0,001$ \\
\hline
\end{tabular}

Tabelle 3 : Aufgeführt ist die Häufigkeitsverteilung der Zeichen und Symptome der Herzinsuffizienz im Patientenkollektiv im Vergleich zwischen eingeschränkter und erhaltener Ejektionsfraktion. 

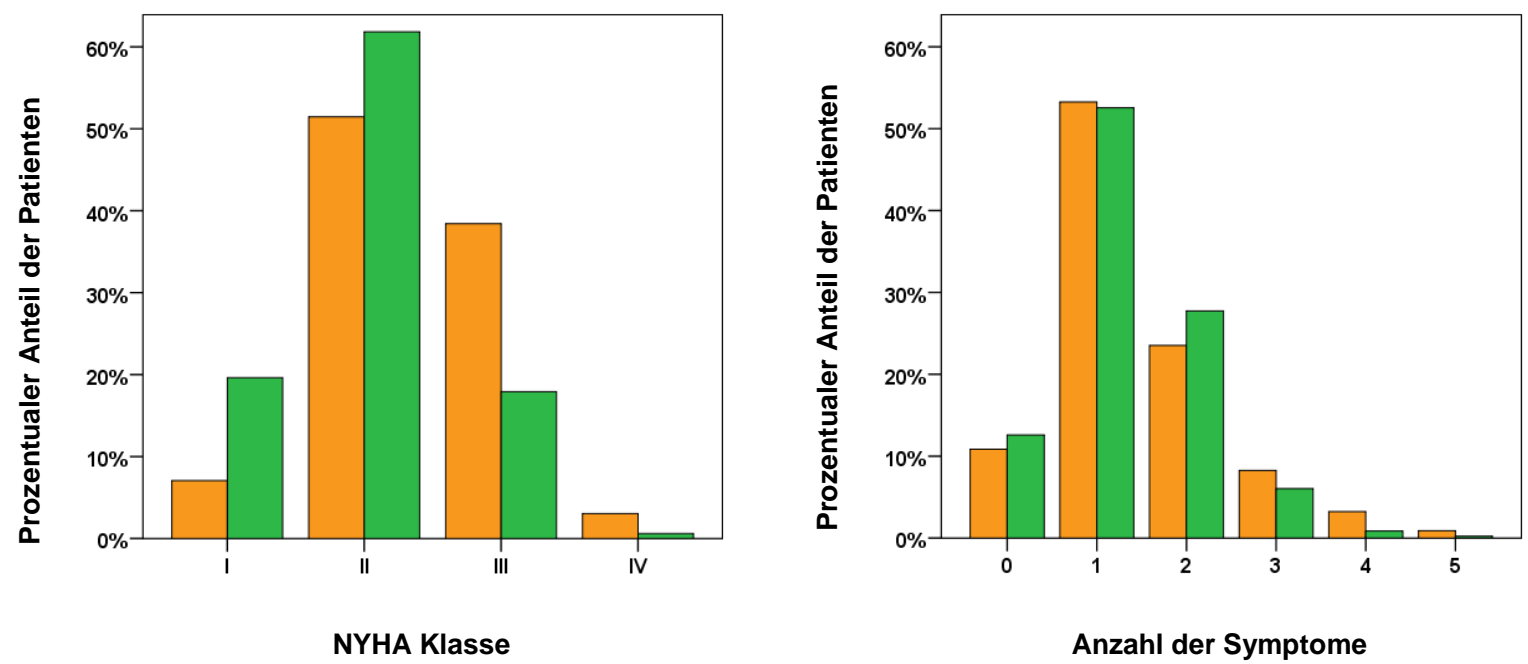

Abbildung 2: Prozentuale Verteilung von NYHA-Klasse und Symptomen der Herzinsuffizienz bei Patienten mit eingeschränkter $(\square$ LVEF<50\%) und bei Patienten mit erhaltener Ejektionsfraktion ( $\square$ LVEF $\geq 50 \%$ ).

In der Abbildung 2 wurde der prozentuale Anteil der Patienten, adjustiert auf die LVEF, entsprechend der jeweiligen NYHA-Klasse und der Anzahl der Symptome aufgetragen.

Die NYHA-Klassen II und III sind im Gesamtkollektiv am häufigsten vertreten, wobei Patienten mit erhaltener Pumpfunktion vergleichsweise weniger symptomatisch sind bzw. die Patienten mit systolischer Herzinsuffizienz höhere NYHA-Klassen aufweisen. In der Anzahl der Symptome unterscheiden sich die Patienten mit HFREF und HFPEF kaum. Die meisten Patienten weisen ein bis zwei Symptome auf. 


\subsubsection{Komorbiditäten im Patientenkollektiv}

In diesem Abschnitt sind Angaben bezüglich der zum Untersuchungszeitpunkt bereits bekannten Komorbiditäten aufgeführt und entsprechend ihrer Verteilung im Gesamtkollektiv bei eingeschränkter und bei erhaltener Ejektionsfraktion aufgelistet.

Insgesamt sind Komorbiditäten sehr häufig: Während sie im Mittel bei 37,2\% der Patienten mit systolischer Herzinsuffizienz vorkommen, so sind bei $33 \%$ der Patienten mit erhaltener Pumpfunktion Komorbiditäten bekannt.

Komorbiditäten wie Diabetes mellitus, Hyperurikämie, renale Dysfunktion, Anämie, COPD, KHK und Hyperlipidämie treten bei Patienten mit systolischer Herzinsuffizienz zahlreicher auf, während Hypertonie und Adipositas bei den Patienten mit erhaltener EF signifikant häufiger vorliegen.

\begin{tabular}{|c|c|c|c|c|c|}
\hline & $\begin{array}{c}\text { LVEF }<50 \\
\% \\
n=2785\end{array}$ & $\begin{array}{c}\text { LVEF<50 } \\
\% \\
\text { Prozent } \\
{[\%]}\end{array}$ & $\begin{array}{c}\text { LVEF } \geq 50 \\
\% \\
n=1294\end{array}$ & $\begin{array}{c}\text { LVEF } \geq 50 \\
\% \\
\text { Prozent } \\
{[\%]}\end{array}$ & p-Wert \\
\hline Diabetes mellitus & 864 & 31,0 & 313 & 24,2 & $<0,001$ \\
\hline Hyperurikämie & 1086 & 39,0 & 252 & 19,5 & $<0,001$ \\
\hline Renale & 962 & 34,5 & 341 & 26,4 & $<0,001$ \\
\hline Dysfunktion & & & & & \\
\hline Anämie & 610 & 21,9 & 183 & 14,1 & $<0,001$ \\
\hline COPD & 421 & 15,1 & 163 & 12,6 & $<0,05$ \\
\hline KHK & 1286 & 46,2 & 405 & 31,3 & $<0,001$ \\
\hline Hypertonie & 1709 & 61,4 & 1014 & 78,4 & $<0,001$ \\
\hline Adipositas & 746 & 26,8 & 486 & 37,6 & $<0,001$ \\
\hline Hyperlipidämie & 1638 & 58,8 & 699 & 54,0 & $<0,01$ \\
\hline
\end{tabular}

Tabelle 4: Die Tabelle zeigt die Häufigkeitsverteilung von Komorbiditäten bei eingeschränkter Ejektionsfraktion im Vergleich zu erhaltener Ejektionsfraktion. 


\subsection{Ergebnisse der ordinalen Regressionsanalyse}

Die folgenden Abbildungen (Abb. 2 bis 12) illustrieren die Überprüfung des Einflusses einzelner Komorbiditäten auf die Ausprägung der Symptome anhand der NYHA-Klasse mittels ordinaler Regressionsanalyse. Die ordinale logistische Regressionsanalyse erfolgte mit der SPSS-Prozedur PLUM. Zur Verdeutlichung zeigt eine Abbildung im Anhang exemplarisch für den Diabetes mellitus ein entsprechendes Output-dokument. In den folgenden Abschnitten erfolgt für die übrigen Komorbiditäten eine analoge Auswertung.

Die Ergebnisse sind als Odds Ratio (OR, 95\% Konfidenzintervall) angegeben. Die Beziehung zwischen NYHA-Klasse und vorliegender Komorbidität wurde auf Alter, Geschlecht und linksventrikuläre Ejektionsfraktion adjustiert. Die NYHA-Klasse war die abhängige Variable und gleichzeitig wurden alle Regressoren eingeschlossen. Getestet wurde, ob der Effekt der Komorbidität in den Untergruppen mit reduzierter und erhaltener Ejektionsfraktion gleich ist. Jedes der folgenden Diagramme zeigt dabei auf der Abszisse die linksventrikuläre Ejektionsfraktion ( $\mathrm{EF}<50 \%, \mathrm{EF} \geq 50 \%$ ), während auf der Ordinate die NYHAKlasse ablesbar ist. Im linken Abschnitt ist der Einfluss der jeweiligen Komorbidität für Männer dargestellt und rechts für Frauen.

Gleichgerichtete Interaktionen bzw. ein vergleichbarer Einfluss auf die NYHA-Klasse bei Herzinsuffizienz mit reduzierter und erhaltener Ejektionsfraktion konnte bei Diabetes mellitus, Hyperurikämie, renaler Dysfunktion und Anämie gezeigt werden. Dabei besteht ein signifikanter Zusammenhang mit einer höheren NYHA-Klasse sowie vergleichbare Auswirkungen bezüglich HFREF und HFPEF ( $p<0,05$ bezüglich der Interaktion mit der LVEF).

Konträre Interaktionen bestehen hingegen bei der Prüfung des Einflusses von COPD, KHK, Adipositas und Hypertonie. So hat eine COPD unterschiedliche Auswirkungen auf die NYHAKlasse bei weiblichen und männlichen Patienten $(p<0,01)$. COPD hat einen stärkeren negativen Effekt auf die NYHA-Klasse der Männer als auf die NYHA-Klasse der Frauen, unabhängig von der linksventrikulären Ejektionsfraktion.

KHK und Adipositas üben einen negativen Einfluss auf die Symptomatik der herzinsuffizienten Patienten mit erhaltener Ejektionsfraktion aus, nicht jedoch auf die Gruppe der Patienten mit eingeschränkter Pumpfunktion $(p \leq 0,01)$.

Eine umgekehrte Wirkung auf die NYHA-Klasse bei Patienten mit HFREF und HFPEF haben das Vorliegen eines Hypertonus oder einer Hyperlipidämie. Hypertonie scheint die NYHAKlasse bei Patienten mit reduzierter Pumpfunktion positiv zu beeinflussen, hat jedoch keinen Effekt auf die Symptomatik oder NYHA-Klasse der Patienten mit erhaltener EF. Darüber 
hinaus variiert der Einfluss einer vorliegenden Hyperlipidämie auf die Patienten mit HFREF und HFPEF.

\section{Diabetes mellitus}

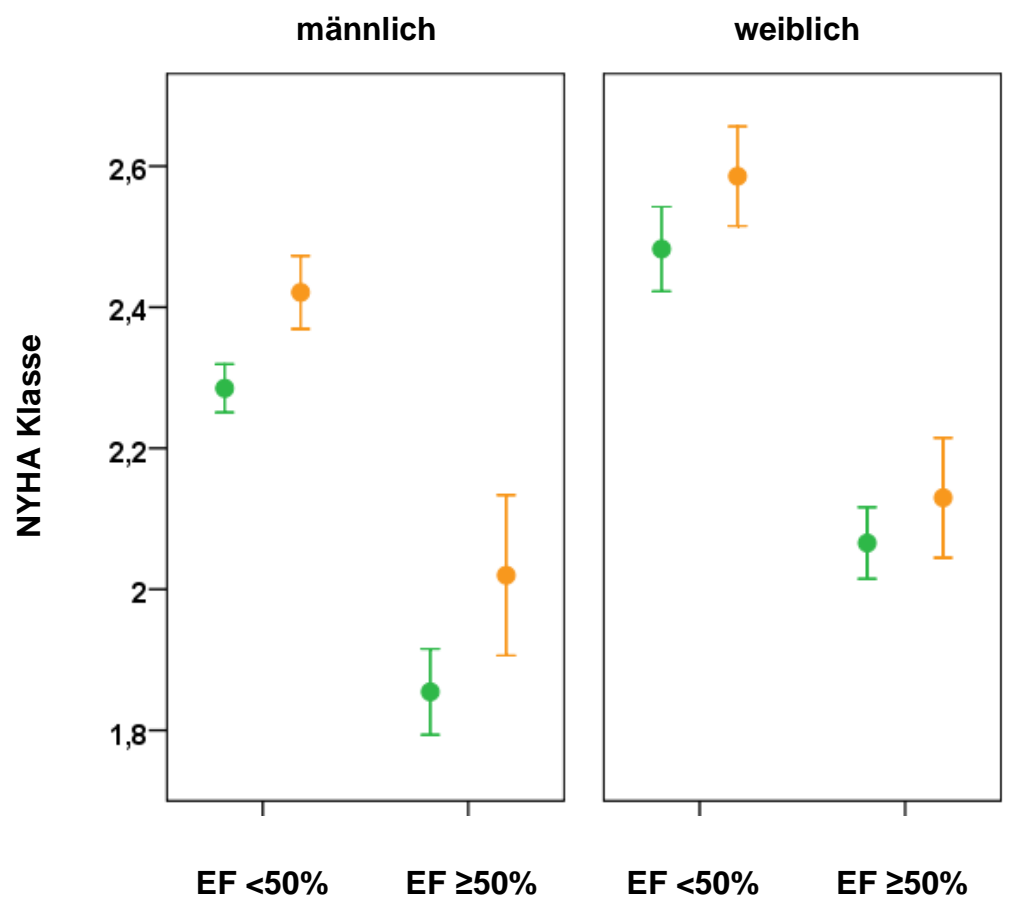

Abbildung 3: Die Abbildung zeigt den Einfluss von Diabetes mellitus ( $\bigcirc$ Diabetes mellitus, kein Diabetes mellitus) auf die NYHA-Klasse bei Patienten mit eingeschränkter Ejektionsfraktion im Vergleich zu Patienten mit erhaltener EF. Ein vorliegender Diabetes mellitus übt einen signifikant negativen Effekt auf die NYHA-Klasse aus (OR=1,26; 95\% $\mathrm{KI}: 1,11-1,45 ; p<0,001)$. Das bedeutet, ein Diabetes mellitus erhöht die Chance (=p/[1-p]), einer höheren NYHA-Klasse zugeordnet zu werden, um 26\%. Allerdings konnten keine unterschiedlichen Auswirkungen zwischen erhaltener und eingeschränkter Ejektionsfraktion auf die Ausprägung der NYHA-Klasse aufgezeigt werden $(p=0,79)$.

Zur Verdeutlichung findet sich im Anhang exemplarisch für den Diabetes mellitus ein entsprechendes Output-Dokument. In den folgenden Abschnitten zu den einzelnen Komorbiditäten wird bei deren Auswertung analog vorgegangen. 


\section{Hyperurikämie}

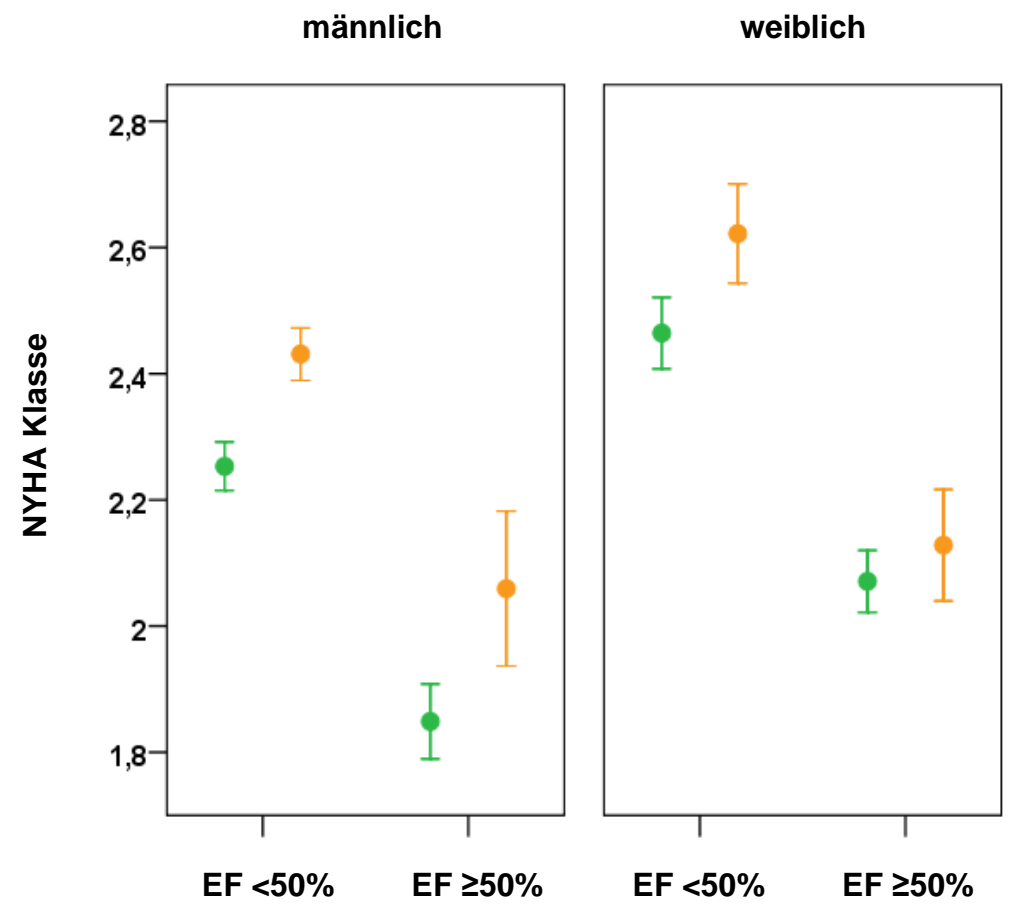

Abbildung 4: Dargestellt ist der Einfluss von Hyperurikämie $(\bigcirc$ Hyperurikämie, $\bigcirc$ keine Hyperurikämie) auf die NYHA-Klasse im Vergleich zwischen reduzierter und erhaltener Ejektionsfraktion. Die NYHA-Klasse wird signifikant negativ von einer Hyperurikämie beeinflusst (OR=1,60; 95\% Kl:1,40-1,82; $\mathrm{p}<0,001)$, jedoch unabhängig von der linksventrikulären Ejektionsfraktion $(p=0,58)$ und vom Geschlecht. 


\section{Renale Dysfunktion}
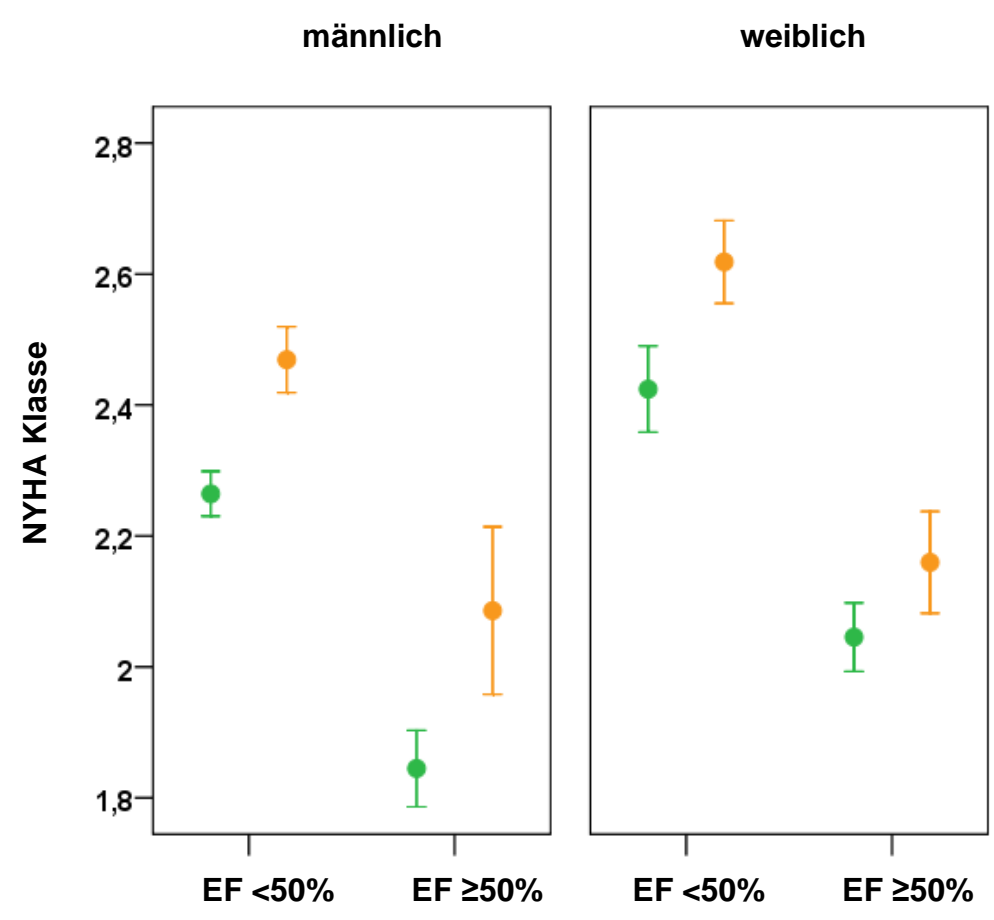

Abbildung 5: Die Abbildung zeigt die Beziehung zwischen renaler Dysfunktion $(\bigcirc$ Renale Dysfunktion, $\bigcirc$ keine renale Dysfunktion) und NYHA-Klasse im Vergleich zwischen eingeschränkter und erhaltener Ejektionsfraktion. Während das Vorhandensein einer renalen Dysfunktion eine signifikant negative Auswirkung auf die NYHA-Klasse hat (OR=1,51; 95\% $\mathrm{Kl}: 1,31-1,73 ; \mathrm{p}<0,001)$, konnte keine signifikante Abhängigkeit von der linksventrikulären Ejektionsfraktion $(p=0,60)$ und vom Geschlecht nachgewiesen werden. 


\section{Anämie}
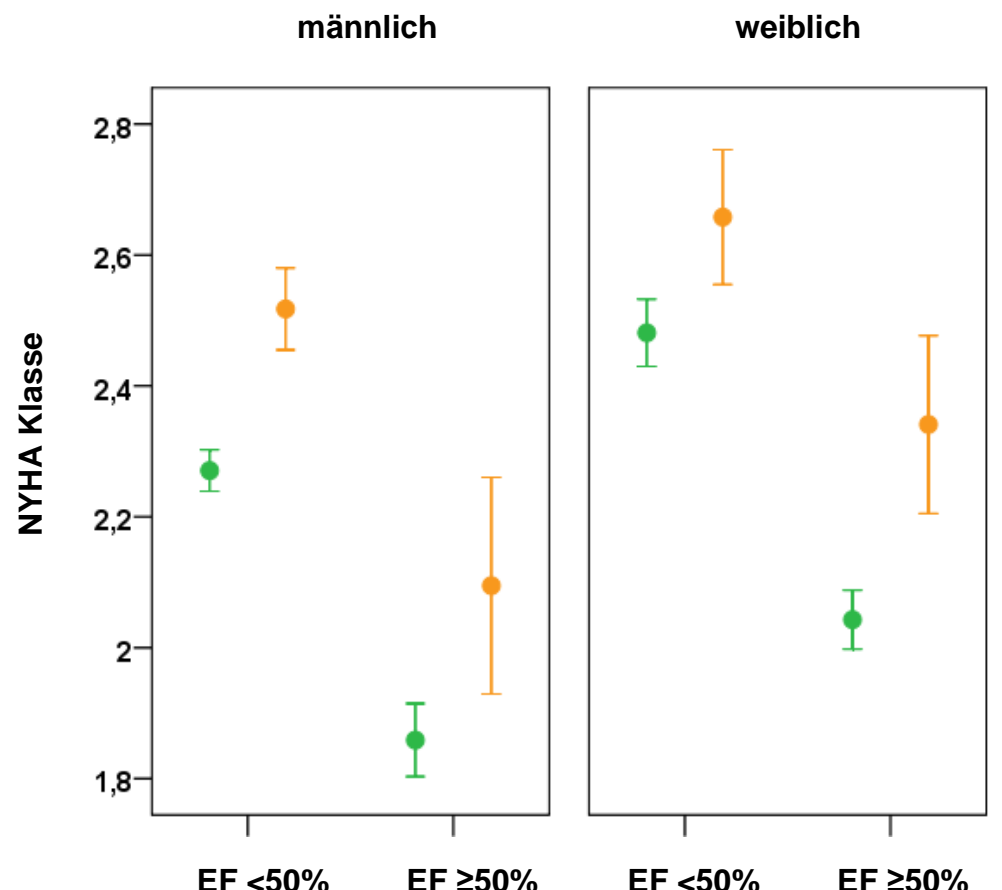

EF $<50 \% \quad E F \geq 50 \%$

Abbildung 6: Abgebildet ist der Einfluss von Anämie ( $\bigcirc$ Anämie, $\bigcirc$ keine Anämie) auf die NYHA Klasse bei reduzierter und erhaltener Ejektionsfraktion. Eine bestehende Anämie hat einen signifikant negativen Effekt auf die NYHA-Klasse (OR=1,77; 95\% KI:1,51-2,07; $p<0,001)$. Jedoch besteht keine Interaktion mit der linksventrikulären Ejektionsfraktion $(p=0,36)$ und auch das Geschlecht zeigt keine signifikanten Auswirkungen auf die NYHAKlasse. 


\section{COPD}

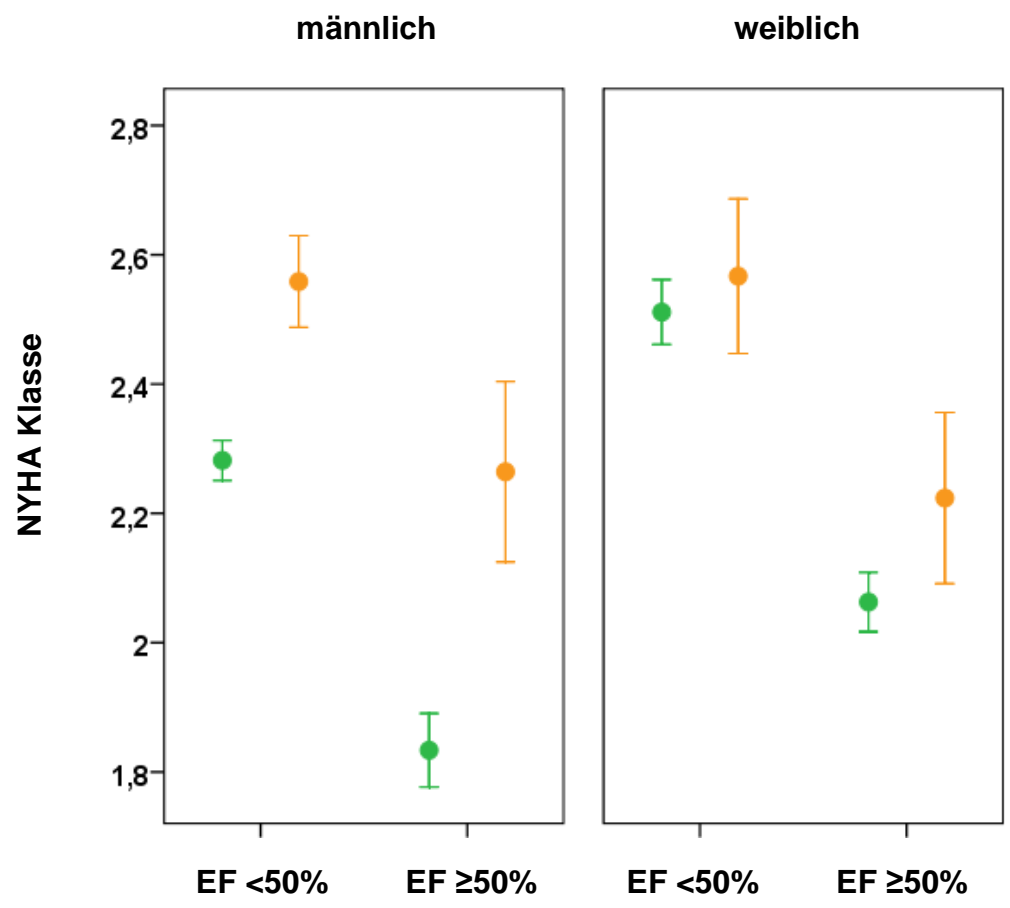

Abbildung 7: Die Abbildung zeigt die Abhängigkeit der NYHA-Klasse von einer COPD $(\bigcirc$ COPD, $\bigcirc$ keine COPD) im Vergleich zwischen eingeschränkter und erhaltener Ejektionsfraktion. Das Vorliegen einer COPD hat unterschiedliche Auswirkungen auf die NYHA-Klasse in Abhängigkeit vom Geschlecht (OR unterscheidet sich bzgl. des Geschlechts; $p<0,01)$. Nur das männliche Geschlecht zeigt einen signifikant negativen Einfluss auf die NYHA-Klasse beim Vorliegen einer COPD (OR=2,25; 95\% KI:1,83-2,77; $p<0,001)$. Bei Frauen konnte kein signifikanter Unterschied bezüglich der NYHA-Klasse in Abhängigkeit von einer COPD nachgewiesen werden (OR=1,36; 95\% KI:0,99-1,88; $p=0,06$ ). Ebenfalls besteht keine Interaktion mit der linksventrikulären Ejektionsfraktion $(p=0,18)$. 


\section{KHK}

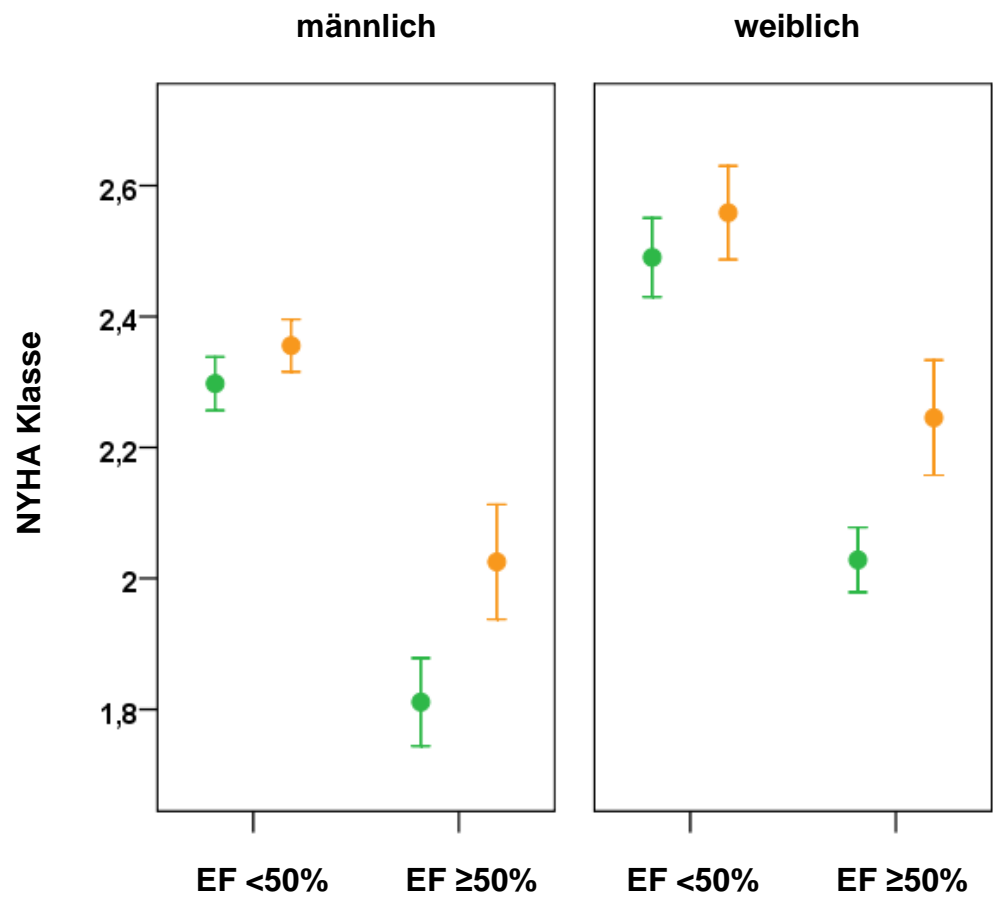

Abbildung 8: Dargestellt ist die Auswirkung von KHK $(\bigcirc \mathrm{KHK}, \bigcirc$ keine KHK) auf die NYHAKlasse bei erhaltener und bei eingeschränkter Ejektionsfraktion. Eine bestehende KHK geht in beiden Gruppen mit verstärkter Luftnot einher, allerdings mit unterschiedlichen Odds Ratios $(p=0,01)$. Eine vorhandene KHK hat nur einen signifikant negativen Einfluss auf die NYHA-Klasse bei Herzinsuffizienz mit erhaltener EF (OR=1,71; 95\% KI:1,33-2,19; $p<0,001)$. Bei der Gruppe mit eingeschränkter EF konnten keine signifikanten Unterschiede bezüglich der NYHA-Klasse gezeigt werden (OR=0,94; 95\% KI:0,80-1,10; $p=0,40)$. 


\section{Adipositas}

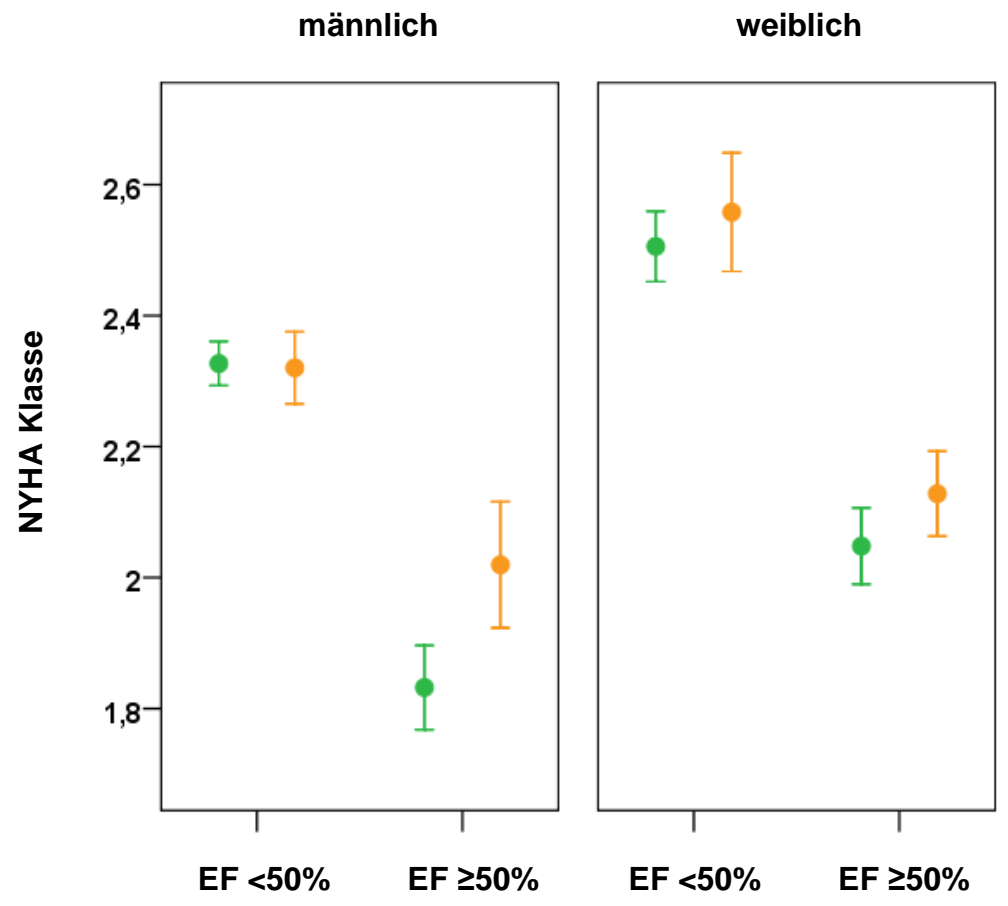

Abbildung 9: Die Abbildung zeigt die Abhängigkeit der NYHA-Klasse von Adipositas ( Adipositas, $\bigcirc$ keine Adipositas) im Vergleich zwischen reduzierter und erhaltener Ejektionsfraktion. Die NYHA- Klasse wird in Anwesenheit von Adipositas und in Abhängigkeit von einer vorliegenden reduzierten oder erhaltenen Pumpfunktion unterschiedlich beeinflusst (unterschiedliche OR für HFREF und HFPEF; $p=0,01$ ). Adipositas hat nur einen signifikant negativen Einfluss auf die NYHA-Klasse bei Herzinsuffizienz mit erhaltener Ejektionsfraktion $(\mathrm{OR}=1,53 ; 95 \% \mathrm{Kl}: 1,22-1,92 ; \mathrm{p}<0,001)$, nicht jedoch auf die Gruppe mit eingeschränkter Pumpfunktion (OR=1,10; 95\% KI:0,93-1,30; $p=0,26)$. 


\section{Hypertonie}

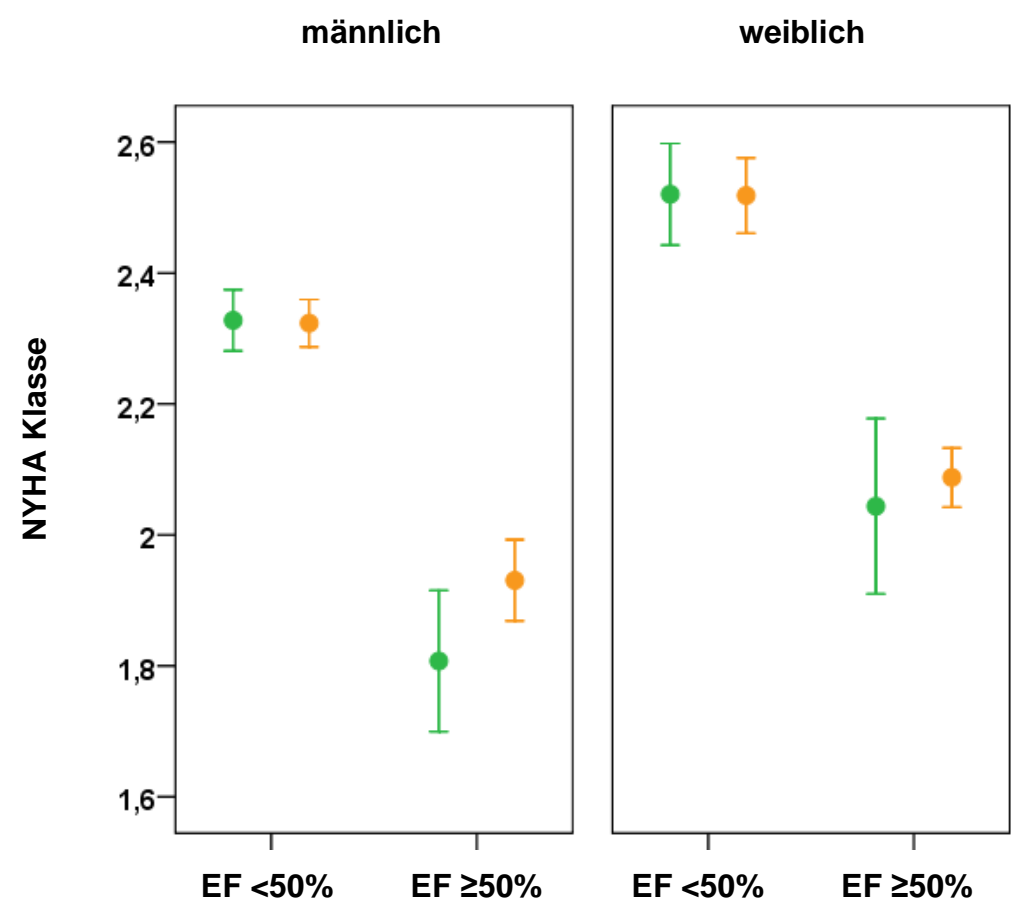

Abbildung 10: Abgebildet ist der Einfluss von Hypertonie $(\bigcirc$ Hypertonie, $\bigcirc$ keine Hypertonie) auf die NYHA-Klasse im Vergleich zwischen erhaltener und eingeschränkter Ejektionsfraktion. Die Odds Ratios für das Vorliegen einer Hypertonie bei LVEF $<50 \%$ und LVEF $\geq 50 \%$ sind möglicherweise unterschiedlich $(p=0,058)$. Daher erfolgt in den Abbildungen 10 und 11 eine genauere Darstellung durch eine Auftrennung der HFREF und HFPEF in festgelegte Altersgruppen. 


\section{Hypertonie bei HFREF}

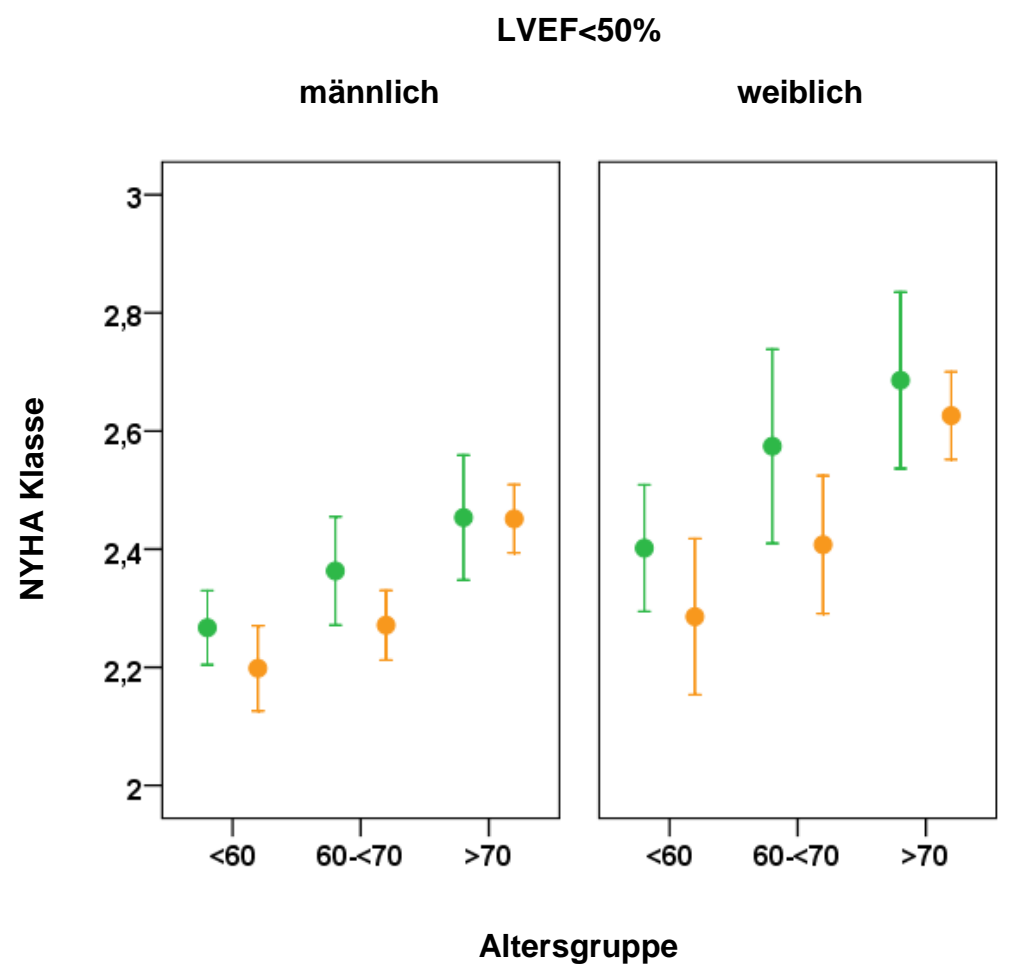

Abbildung 11: Die Abbildung zeigt den Einfluss von Hypertonie ( $\bigcirc$ Hypertonie, $\bigcirc$ keine Hypertonie) auf die NYHA-Klasse bei eingeschränkter Pumpfunktion, adjustiert auf Geschlecht und festgelegte Altersgruppen. Eine vorliegende Hypertonie geht mit einer besseren NYHA-Klasse einher und scheint somit die Symptomatik bei herzinsuffizienten Patienten mit eingeschränkter Ejektionsfraktion positiv zu beeinflussen (OR=0,75; 95\% $\mathrm{Kl}: 0,64-0,88 ; \mathrm{p}=0,001)$. 


\section{Hypertonie bei HFPEF}

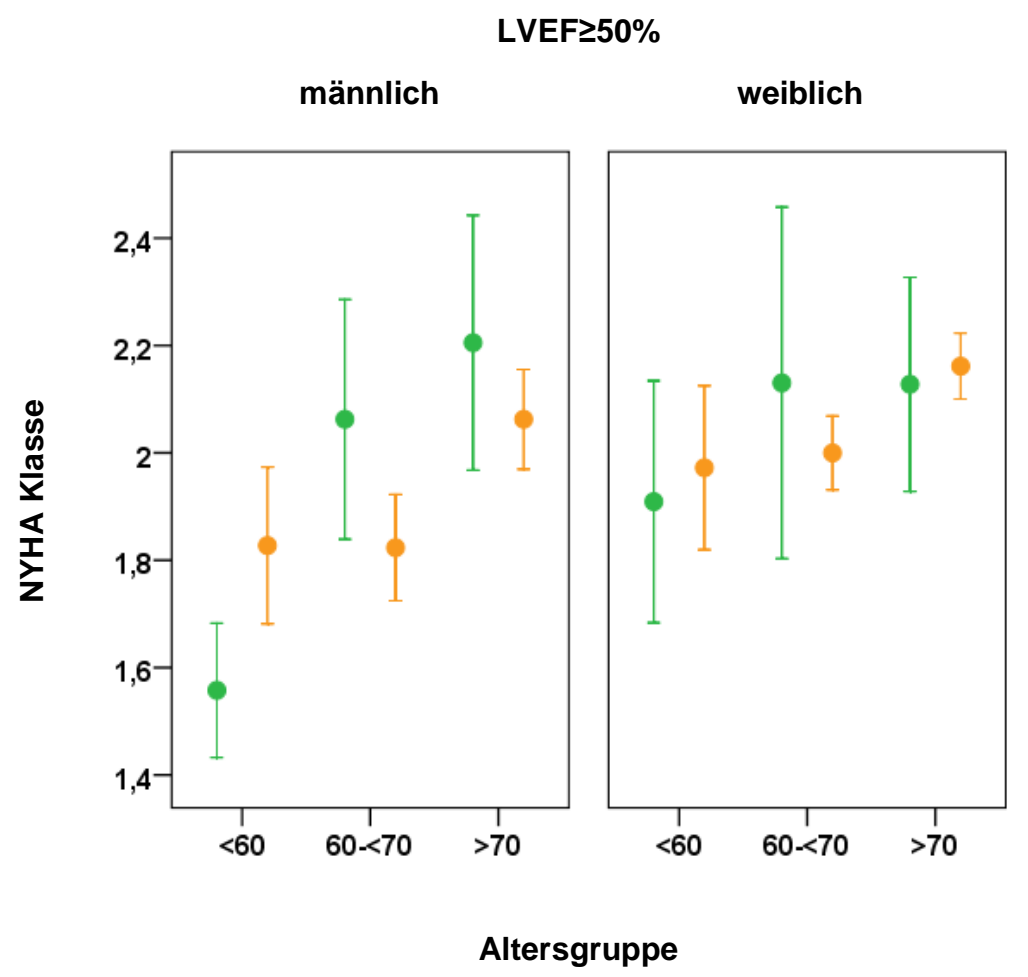

Abbildung 12: Dargestellt ist der Einfluss von Hypertonie $(\bigcirc$ Hypertonie, $\bigcirc$ keine Hypertonie) auf die NYHA-Klasse bei erhaltener Pumpfunktion, adjustiert auf Geschlecht und definierte Altersgruppen. Ein vorhandener Hypertonus hat keinen Einfluss auf die Symptomatik oder NYHA-Klasse bei herzinsuffizienten Patienten mit erhaltener Pumpfunktion (OR=0,89; 95\% Kl:0,66-1,18; $p=0,41)$. 


\section{Hyperlipidämie}

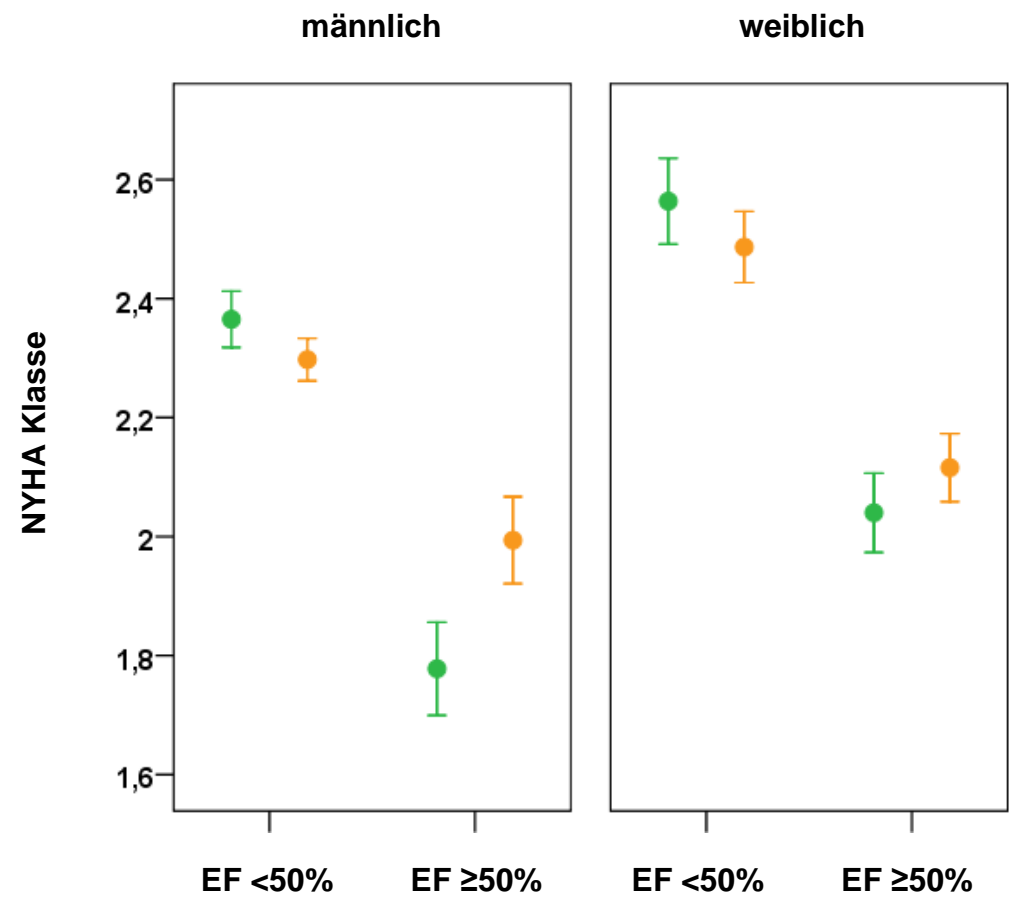

Abbildung 13: Die Abbildung zeigt den Zusammenhang zwischen Hyperlipidämie $(\bigcirc$ Hyperlipidämie, $\bigcirc$ keine Hyperlipidämie) und NYHA-Klasse bei reduzierter und bei erhaltener Ejektionsfraktion. Der Einfluss einer Hyperlipidämie auf die Patienten mit HFREF und HFPEF variiert (verschieden ORs für HFREF und HFPEF; $p<0,001$ ). Eine bestehende Hyperlipidämie hat bei vorliegender eingeschränkter Ejektionsfraktion einen positiven Einfluss auf die NYHA-Klasse (OR=0,75; 95\% KI: 0,65-0,87; $\mathrm{p}<0,001)$ und einen eher negativen Effekt auf die NYHA-Klasse bei erhaltener Pumpfunktion (OR=1,38; 95\% KI:1,11$1,73 ; p<0,005)$. 


\subsection{Ergebnisse der multivariaten Analyse bei HFREF und HFPEF}

Die folgenden Abbildungen zeigen multiple ordinale logistische Regressionsrechnungen von allen Komorbiditäten, die einzeln einen signifikanten Einfluss auf die NYHA-Klasse aufweisen, anhand derer die Beständigkeit des Zusammenhangs, auch in Abhängigkeit von anderen Faktoren wie zur Erkrankung führender morphologischer Parameter, überprüft werden soll. Ergänzend wurden zwei echokardiographische Parameter eingefügt: Die linksventrikuläre Ejektionsfraktion (LVEF), die den Schweregrad der systolischen Dysfunktion bei HFREF bezeichnet, und der linksventrikuläre enddiastolische Diameter (LVEDD), der die gestörte ventrikuläre Füllung bei HFPEF widerspiegelt. Dies erlaubt es, den Einfluss der ventrikulären Dysfunktion und der Komorbiditäten auf die Symptome abzubilden. Ein entsprechendes SPSS-Outputdokument, jedoch ohne Einbezug der echokardiographischen Parameter, befindet sich exemplarisch im Anhang.

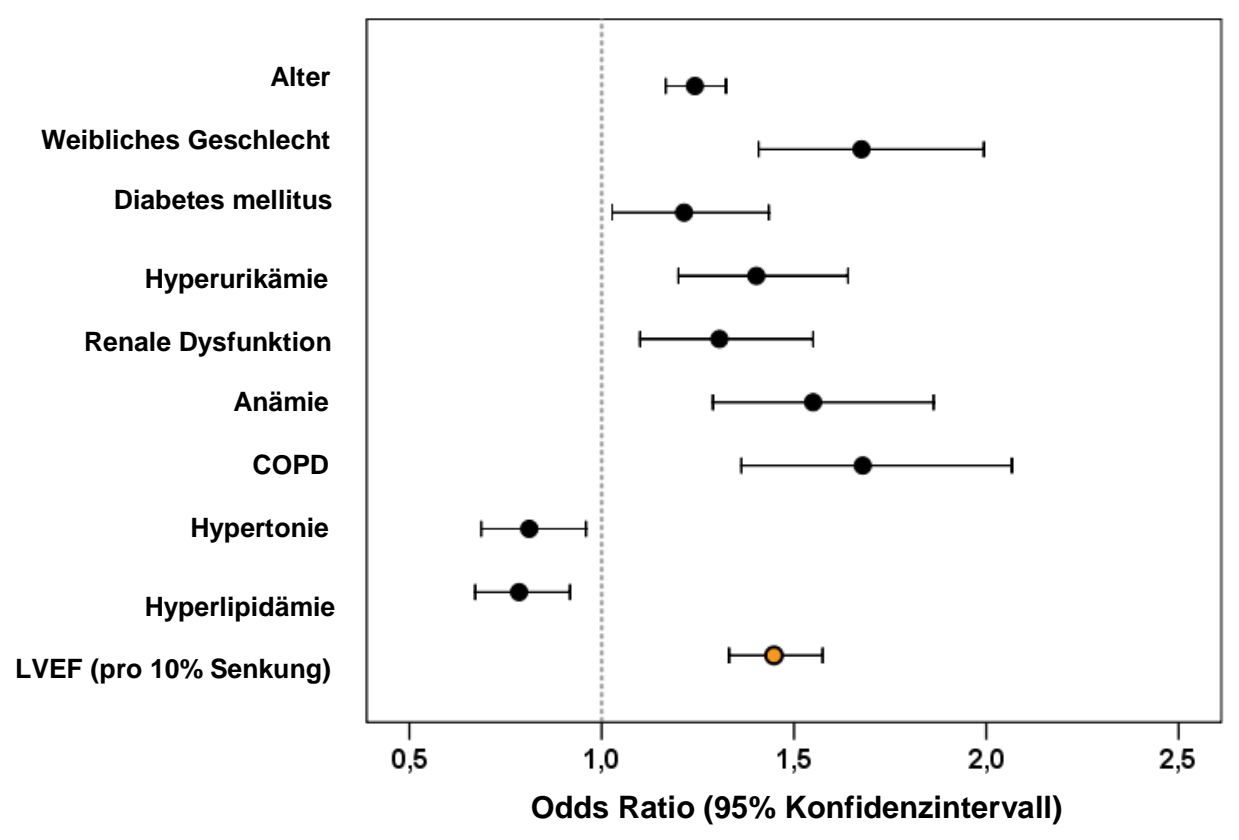

Abbildung 14: Diese Abbildung zeigt die multivariate Analyse, d.h. eine multiple ordinale logistische Regressionsrechnung, des Einflusses der einzelnen Komorbiditäten auf die Ausprägung der Symptomatik bei herzinsuffizienten Patienten mit reduzierter Ejektionsfraktion. Die Abszisse zeigt das Ausmaß des Einflusses als Odds Ratio (OR, 95\% Konfidenzintervall). Auf der Ordinate sind die einzelnen Komorbiditäten aufgetragen. 
Zusätzlich zu den Komorbiditäten wird der Einfluss der linksventrikulären Ejektionsfraktion pro $10 \%$ Abfall geprüft.

Es wird deutlich, dass Alter, weibliches Geschlecht, Diabetes mellitus, Hyperurikämie, renale Dysfunktion, Anämie, COPD und ein Abfall der LVEF einen signifikant negativen Einfluss auf die Symptomatik und die NYHA-Klasse bei HFREF haben. Die Abhängigkeit der NYHAKlasse von der LVEF entspricht dabei in etwa dem Vorliegen einer Hyperurikämie oder renalen Dysfunktion. Der LVD(ED) zeigte in der univariaten Analyse keinen signifikanten Effekt und wurde daher hier nicht aufgeführt.

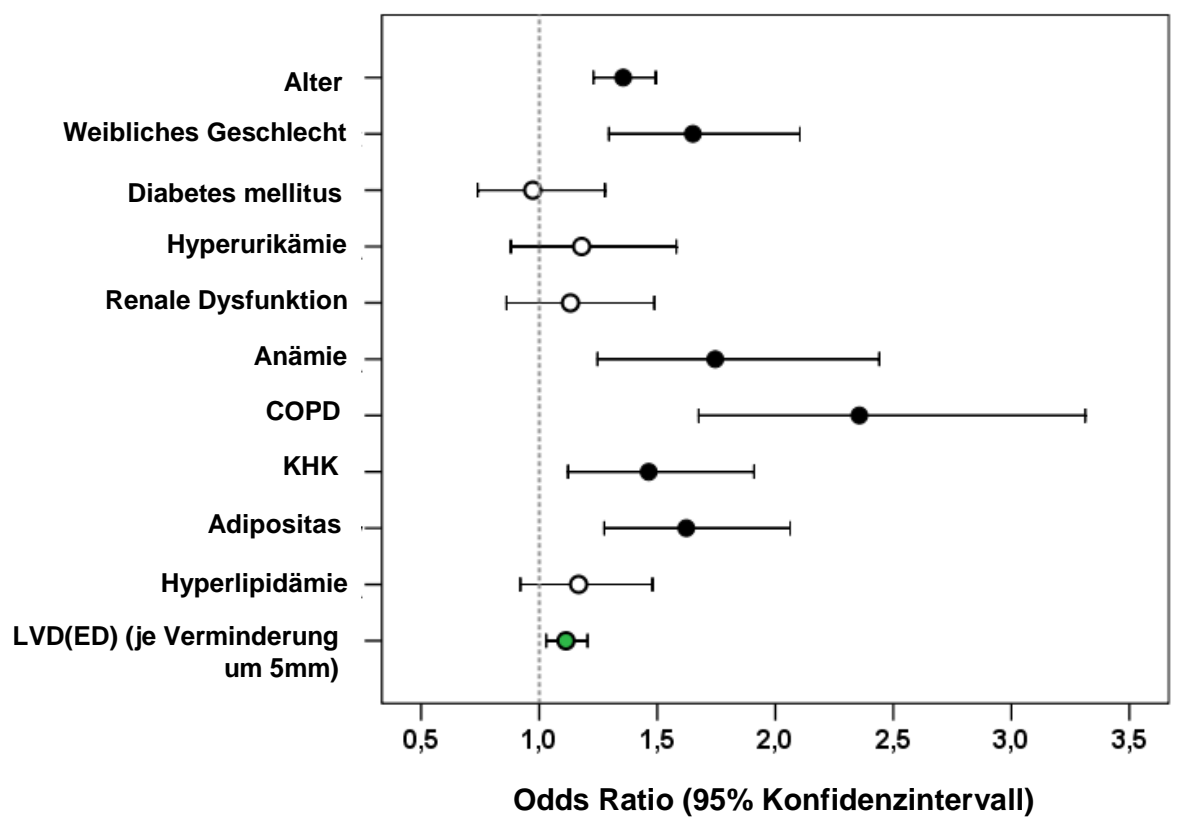

Abbildung 15: Dargestellt ist die multivariate Analyse der Abhängigkeit der NYHA- Klasse von Komorbiditäten bei Herzinsuffizienz mit erhaltener Ejektionsfraktion. Auch hier sind auf der Abszisse die Odds Ratio (OR, 95\% Konfidenzintervall) und auf der Ordinate die einzelnen Komorbiditäten angegeben. Darüber hinaus ist der Einfluss einer Abnahme des enddiastolischen linksventrikulären Durchmessers (LVD(ED)) um $5 \mathrm{~mm}$ geprüft und ebenfalls auf der Ordinate aufgelistet.

Ein signifikant negativer Effekt auf die NYHA-Klasse in der Gruppe der HFPEF besteht nur durch das Alter, das weibliche Geschlecht, Anämie, COPD, KHK, Adipositas und eine Abnahme des LVD(ED). 
Allerdings wird deutlich, dass der Einfluss der Komorbiditäten auf die NYHA- Klasse höher ist, als Veränderungen in relevanten linksventrikulären Parametern wie dem LVD(ED). Die EF wurde hier nicht aufgeführt, da in den univariaten Analysen keinen signifikanter Effekt gezeigt werden konnte. 


\section{Diskussion}

Die Inzidenz sowie die Prävalenz der chronischen Herzinsuffizienz sind hoch und wachsen mit steigendem Alter. Vor allem die Prävalenz der Herzinsuffizienz mit erhaltener Ejektionsfraktion, die etwa die Hälfte der Patientenpopulation mit chronischer Herzinsuffizienz aufweist, nimmt besonders wegen der immer älter werdenden Bevölkerung stetig zu. Während die Epidemiologie, Therapie und Prognose der systolischen Herzinsuffizienz extensiv untersucht und beschrieben worden sind, sind Studienergebnisse zur Herzinsuffizienz mit erhaltener Ejektionsfraktion limitiert und teilweise widersprüchlich.

Durch eine standardisierte, evidenzbasierte und medikamentöse Therapie (ACE- Hemmer, AT1-Rezeptorblocker, Betablocker und Aldosteronantagonisten) der chronischen Herzinsuffizienz mit reduzierter Ejektionsfraktion konnte eine Verbesserung von Mortalität und Morbidität dieser Patienten nachgewiesen werden.

Im Gegensatz dazu erfolgt die Therapie der Herzinsuffizienz mit erhaltener EF derzeit noch theoretisch und empirisch. Grund hierfür ist das Fehlen erfolgreicher, randomisierter, doppelblinder Multizenterstudien. So konnte bei einer Therapie mit Perindopril (PEP-CHF, Cleland et al. 2006), Digoxin (Ahmed et al. 2006) oder Irbesartan (I-Preserve, Massie et al. 2008) keine signifikante Reduktion der Mortalität oder Morbidität herzinsuffizienter Patienten mit erhaltener EF nachgewiesen werden. Die CHARM-Preserved-Studie (Yusuf et al. 2003) konnte eine Reduktion der Hospitalisierungsraten im Rahmen einer Herzinsuffizienz durch Candesartan zeigen, aber diese Ergebnisse weisen trotz großer Patientenzahl nur eine moderate Signifikanz auf. Nur die SENIORS-Studie (Flather et al. 2005) ergab eine effektive Reduktion von Morbidität und Mortalität der über 70-jährigen Patienten unter der Therapie von Nebivolol unabhängig von der Ejektionsfraktion. Damit ist derzeit keine evidenzbasierte Therapie vorgegeben und deshalb steht eine Risikofaktor kontrollierte Behandlung im Vordergrund.

Während in einigen Studien bereits bewiesen worden ist, dass vorhandene Komorbiditäten die Prognose und Morbidität herzinsuffizienter Patienten mit reduzierter Ejektionsfraktion negativ beeinflussen, ist der Einfluss dieser auf Herzinsuffizienz mit erhaltener EF bislang unzureichend untersucht. Besonders deren Stellenwert bei der Diagnosefindung und ihre Rolle als therapeutischer Angriffspunkt sind bisher unklar. Das ist möglicherweise auch ein Grund für das Versagen von großen Studien.

Zu diesem Zweck untersucht die vorliegende Arbeit die Verteilung typischer Komorbiditäten in einem großen Herzinsuffizienzkollektiv und stellt deren unterschiedliche Auswirkungen auf die Symptomatik bei erhaltener sowie eingeschränkter Ejektionsfraktion dar.

Im Folgenden sollen die Ergebnisse der vorliegenden Arbeit bewertet und in den bisherigen Wissensstand der Forschung zu diesem Thema eingearbeitet werden. 


\subsection{Studiendesign}

Für die Interpretation einer Studie und die daraus resultierende Aussagekraft der Ergebnisse ist das Studiendesign von Bedeutung. Bezogen auf die hier vorliegende Arbeit ist es wichtig, repräsentative Ergebnisse zu erzielen und zu verhindern, dass diese durch einen Zufall zustande gekommen sein könnten. Daher ist es sinnvoll große Patientenkollektive und ein breites Patientenspektrum zu wählen, um so ein homogenes Bild schaffen zu können. Ebenso werden die Analysen noch aussagekräftiger, wenn ähnliche Ergebnisse in verschiedenen Studien durch unterschiedliche Untersucher und $\mathrm{zu}$ verschiedenen Zeiten erzielt werden und diese einzelnen Studien durch verschiedene Designs zustande kommen.

In dieser Studie sind alle verfügbaren Teilprojekte des deutschen multizentrischen Kompetenznetzes Herzinsuffizienz (KNHI) auf Patienten untersucht worden, die für unsere Studie in Frage kommen könnten, um so ein möglichst großes Patientenkollektiv zu erstellen. Dementsprechend sind 8268 Patienten aus elf Teilprojekten des KNHI, die im Zeitraum von 2003 bis 2008 durchgeführt wurden, auf das Vorliegen einer chronischen Herzinsuffizienz untersucht worden. Letztendlich konnten von 4259 chronisch herzinsuffizienten Patienten 4079 Patienten für die Analysen dieser Studie „Einfluss von typischen Komorbiditäten auf die Ausprägung der Symptomatik bei Herzinsuffizienz mit eingeschränkter und erhaltener LV-Funktion“ einbezogen werden.

Die elf ausgewerteten Projekte beinhalten neben epidemiologischen Teilprojekten auch einige Therapiestudien. Dadurch ist zwar eine reine epidemiologische Beurteilung limitiert, verschafft uns aber ein breiteres und repräsentativeres Bild.

Da die Rekrutierung aus Teilprojekten des $\mathrm{KNHI}$ erfolgt ist, unterliegt diese einer gewissen Vorselektion. Jedes Teilprojekt zeichnet sich durch sein eigenes Design und individuell festgelegte Ein- und Ausschlusskriterien aus. Aber bei allen unseren Patienten wurde die Diagnose der chronischen Herzinsuffizienz durch einen Kardiologen gestellt. Außerdem wurde bei allen eine Echokardiographie durchgeführt und anhand der echokardiographisch festgelegten LVEF wurde das Patientenkollektiv entsprechend eines Cut-Off-Wertes von $50 \%$ nach chronischer Herzinsuffizienz mit eingeschränkter (HFREF) und erhaltener (HFPEF) Ejektionsfraktion eingeteilt. Die Herzinsuffizienzsymptomatik wurde anhand geeigneter Kriterien erfasst: neben der NYHA-Klassifizierung wurden bei allen Patienten zusätzlich die Framinghamkriterien erhoben.

Durch das Betrachten des Gesamtbildes dieser verschiedenen genutzten Kollektive und das Zusammenkommen unterschiedlicher Ein- und Ausschlusskriterien entsteht ein homogenes Bild und breitere Erkenntnisse werden ermöglicht.

Im Vergleich dazu sind frühere epidemiologische und therapeutische Studien durch ihre Einund Ausschlusskriterien genau definiert und jede dieser Studien hat ihr eigenes Design. 
Dadurch, dass z.B. in einigen Studien nur ambulante oder nur hospitalisierte Patienten beobachtet wurden, bei einigen die Diagnose der chronischen Herzinsuffizienz schon bestand oder erst im Verlauf festgestellt wurde oder dass eine Einteilung in verschiedene Altersgruppen erfolgte etc., sind diese Studien untereinander kaum vergleichbar und führen zu einem inhomogenen Bild des Patientenkollektivs. So haben Vasan et al. (1999) echokardiographische Untersuchungen eines ebenfalls vorselektierten Patientenkollektivs mit bekannter Herzinsuffizienz der Framingham Heart Study ausgewertet und diese einem dem Geschlecht und Alter angepassten Kontrollkollektiv gegenübergestellt. Smith et al. (2003) haben ein Kollektiv nur aus hospitalisierten Patienten, die Merkmale einer bestehenden Herzinsuffizienz aufwiesen, untersucht. Schließlich haben Bursi et al. (2006) Patienten mit klinischen Zeichen einer Herzinsuffizienz aus einem elektronisch medizinischen Datensatz in Olmsted County identifiziert. In zurückliegenden Therapiestudien zur chronischen Herzinsuffizienz mit erhaltener Ejektionsfraktion sind Patienten untersucht und rekrutiert worden, bei denen entweder bereits die Diagnose einer chronischen Herzinsuffizienz bestand, die nur die Symptome einer solchen aufwiesen oder bei denen bereits eine oder mehrere Hospitalisierungen wegen Herzinsuffizienz oder anderen kardialen Ursachen stattgefunden hatte. Diese Therapiestudien haben die Wirksamkeit von Placebo und beispielsweise Candesartan (CHARM-Preserved, Yusuf et al. 2003), Nebivolol (SENIORS, Flather et al. 2005), Perindopril (PEP-CHF, Cleland et al. 2006), Digoxin (Ahmed et al. 2006) oder Irbesartan (I-Preserve, Massie et al. 2008) miteinander verglichen.

Im Gegensatz zu unserer Studie setzten sich vor allem die epidemiologischen Studien aus viel kleineren Kollektiven von Erkrankten zusammen. Die Therapiestudien bestanden zwar meist aus großen Kollektiven, aber sie sind dadurch in ihrer Aussagekraft und Vergleichbarkeit limitiert, dass sie sich entweder auf die HFREF oder die HFPEF beschränken.

Ein weiteres Problem der Epidemiologie- und Therapiestudien ist, dass verschiedene CutOff-Werte der LVEF genutzt wurden und dadurch unterschiedliche Definitionen bezüglich einer HFREF oder HFPEF entstanden sind. Wie in der hier vorliegenden Arbeit legten auch Vasan et al. (1999) und Bursi et al. (2006) den Grenzwert der LVEF auf 50\% fest. Auch Paulus et al. nutzen eine EF von 50\% als Cut-Off-Wert und etablierten diese in den Leitlinien als Standart zur Diagnosestellung einer diastolischen Herzinsuffizienz (Paulus et al. 2007). Im Gegensatz dazu definierten Ahmed et al. (2006) und Massie et al. (I-Preserve 2008) das Vorliegen einer Herzinsuffizienz mit erhaltener Pumpfunktion bei einer ermittelten Ejektionsfraktion $>45 \%$, während Smith et al. (2003) und Yusuf et al. (CHARM-Preserved 2003) den Cut off für die LVEF auf $>40 \%$ legten. Die Wahl eines so niedrigen Grenzwertes für die LVEF hat zur Folge, dass unter den herzinsuffizienten Patienten mit erhaltener EF mehr Patienten mit schon eingeschränkter Pumpfunktion zu finden sind. Dies wirkt sich 
beispielsweise auf die Ausprägung der Symptomatik und die Altersverteilung aus und nimmt somit Einfluss auf die Verteilung der Basischarakteristika der Patienten.

Frühere epidemiologische und therapeutische Studien weisen also durch definierte Ein- und Ausschlusskriterien sehr spezielle Designs auf. Dadurch sind sie untereinander kaum vergleichbar und besitzen nur eine begrenzte Aussagekraft. Im Gegensatz dazu fasst unsere Studie die verschiedenen Designs zusammen, ermöglicht uns so ein breites und umfassendes Bild.

\subsection{Patientenkollektiv}

Für die vorliegende Arbeit sind 4079 herzinsuffiziente Patienten untersucht worden. Dabei überwogen männliche Patienten mit 65,6\%. Auch in einigen früheren epidemiologischen Studien war eine leichte Dominanz des männlichen Geschlechts erkennbar, allerdings bei einem viel kleineren Datensatz. So liegt der Anteil der Männer bei Vasan et al. (1999) bei $54,8 \%$ von insgesamt 73 Patienten und bei Smith et al. (2003) sind 52\% der 413 Patienten männlichen Geschlechts. Nur bei Bursi et al. (2006) besteht mit 50\% Männern von 556 Patienten eine gleiche Verteilung bezüglich des Geschlechts. Unsere Studie beinhaltet neben den epidemiologischen auch therapeutische Teilprojekte. Da die Therapiestudien verglichen mit Epidemiologiestudien häufig einen größeren Männeranteil aufweisen, ist auch in der vorliegenden Arbeit das männliche Geschlecht prozentual häufiger vertreten. Insgesamt ist unser Patientenkollektiv bezüglich der Geschlechterverteilung mit den früheren Studien also gut vergleichbar.

Die Patienten dieser Studie sind anhand ihrer LVEF klassifiziert worden. Die Gruppe der Patienten mit Herzinsuffizienz und eingeschränkter Ejektionsfraktion $(E F<50 \%)$ macht mit insgesamt 2785 Patienten (68,3\%) den größeren Anteil aus, während eine erhaltene Ejektionsfraktion (EF $\geq 50 \%$ ) nur bei 1294 Patienten nachgewiesen werden konnte. Dass die HFREF überwiegt lässt sich dadurch erklären, dass sich die Teilprojekte häufiger mit der systolischen Herzinsuffizienz befasst haben. Außerdem haben wir durch einige therapeutische Teilprojekte einen größeren Männeranteil verglichen mit rein epidemiologischen Studien. Da die HFPEF insgesamt häufiger bei Frauen beobachtet wird, was sich auch in der hier vorliegenden Studie zeigt, könnte dies ein weiterer Grund für die ungleiche Verteilung zwischen HFREF und HFPEF sein. Vasan et al. (1999) und Bursi et al. (2006), deren LVEF-Cut-Off ebenfalls bei 50\% liegt, haben mit einem Anteil von 51\% herzinsuffizienter Patienten mit erhaltener EF bei Vasan et al. (1999) und 55\% bei Bursi et al. (2006) eine annährend gleiche Verteilung zwischen HFREF und HFPEF gezeigt. Auch bei 
Smith et al. (2003), deren Grenzwert der LVEF auf 40\% festgelegt wurde, entspricht ein Anteil von 52\% HFPEF etwa der Normalverteilung in der Bevölkerung.

Betrachtet man nun die Verteilung des Geschlechts entsprechend der LVEF, so überwiegt die Zahl der Patienten männlichen Geschlechts bei der systolischen Herzinsuffizienz in der vorliegenden Studie mit 74,5\% deutlich, während das weibliche Geschlecht mit 53,8\% häufiger bei HFPEF vertreten war. Ebenso wiesen Vasan et al. (1999) mit 64,8\%, Smith et al. (2003) mit 63\% und Bursi et al. (2006) mit 57\% einen höheren Prozentsatz an Frauen in der Gruppe der Herzinsuffizienz mit erhaltener Ejektionsfraktion und eine deutliche Mehrheit der systolischen Herzinsuffizienz bei Männern auf (75\% bei Vasan et al., $65 \%$ Smith et al., 58\% Bursi et al.). Eine ähnliche Verteilung findet man bei der Therapiestudie PEP-CHF (Cleland et al. 2006) mit einem Frauenanteil von 55\% und bei der I-Preserve-Studie (Massie et al. 2008) mit insgesamt 60\% Frauen. Die CHARM-Preserved-Studie (Yusuf et al. 2003) zeigt im Vergleich zum CHARM-Program insgesamt einen größeren Frauenanteil. In diesen Arbeiten konnte also eine Präferenz unter herzinsuffizienten Patienten mit erhaltener Pumpfunktion zugunsten des weiblichen Geschlechts nachgewiesen werden. Eine Verteilung zugunsten des männlichen Geschlechts findet man nur in der SENIORS-Studie (Flather et al. 2005) mit 63\% und bei Ahmed et al. (2006) mit 59\%. Die Geschlechterverteilung in den genannten Epidemiologie- und Therapiestudien entspricht unserem Patientenkollektiv und macht es somit repräsentativ.

Bezüglich des Alters fällt in der vorliegenden Studie auf, dass die Patienten mit erhaltener Ejektionsfraktion mit einem Mittelwert von 67(₫13) Jahren älter waren als das Patientenkollektiv mit reduzierter Ejektionsfraktion, die ein mittleres Alter von 63( \pm 14$)$ Jahren aufwiesen. Auch bei Smith et al. (2003) mit einem mittleren Alter von 73( \pm 11$)$ Jahren bei HFPEF und 70( \pm 11$)$ Jahren bei HFREF sowie bei Bursi et al. (2006) mit einem mittleren Alter von 77,4 bei HFPEF und 73,4 Jahren bei HFREF konnte gezeigt werden, dass die Patienten mit einer erhaltenen EF älter waren. Bei Vasan et al. (1999) waren dagegen die Patienten mit systolischer Herzinsuffizienz älter (72 \pm 9 Jahre bei HFPEF und $74 \pm 7$ Jahre bei HFREF). Allerdings beziehen sich die Studien von Vasan et al. auf ein viel kleineres Patientenkollektiv. Deren Ergebnisse sind somit weniger repräsentativ.

Insgesamt war das mittlere Alter bei den epidemiologischen Studien höher als in der hier vorliegenden Arbeit. Die einzelnen Studien sind jedoch immer durch Ein- und Ausschlusskriterien definiert und beschränken sich meist auf die Untersuchung einer bestimmten Altersgruppe. Unsere Arbeit besteht aus mehreren Teilprojekten mit verschiedenen Altersgruppen und beinhaltet neben epidemiologischen auch therapeutische Studien, die sich auch in ihrer Altersverteilung unterscheiden. Denn wie beispielsweise bei CHARM-Preserved mit einem mittleren Alter von 67,2 Jahren und bei Ahmed et al. (2006) 
mit 67 Jahren zu erkennen ist, sind die Therapiestudien häufig durch einen niedrigeren Mittelwert bezüglich des Alters gekennzeichnet. Die SENIORS-Studie (Flather et al. 2005) sowie PEP-CHF (Cleland et a. 2006) können hier nicht in den Vergleich einbezogen werden, da ein Einschlusskriterium das Mindestalter von 70 Jahren war.

Insgesamt ist mit unserer Studie zwar kein rein epidemiologischer Vergleich möglich, aber die hier vorliegende Arbeit weist durch die Untersuchung verschiedener Teilprojekte ein sehr repräsentatives Patientenkollektiv auf, bei dem neben einer großen Patientenzahl auch ein viel breiteres Patientenspektrum erfasst, untersucht und dargestellt werden konnte.

Bezüglich der Vormedikation zeigen sich zwischen der Gruppe herzinsuffizienter Patienten mit reduzierter und erhaltener Pumpfunktion deutliche Unterschiede, die nicht gut miteinander vergleichbar sind. Denn bei Patienten mit systolischer Herzinsuffizienz erfolgt eine medikamentöse Behandlung der Herzinsuffizienz und der daraus hervorgehenden Symptomatik. Diese Therapie basiert auf den Leitlinien und erfolgt meist in einer ViererKombination, wie auch in der vorliegenden Arbeit deutlich wird: ACE-Hemmer bzw. AT1Rezeptorblocker wurden von 88\% der Patienten, Betablocker von 84\% und Diuretika von 80\% eingenommen. Der Gebrauch von Aldosteron-Antagonisten ist mit 46\% deutlich geringer ausgefallen, ist aber laut den Leitlinien erst ab einer EF von $\leq 35 \%$ indiziert.

Für die Herzinsuffizienz mit erhaltener Ejektionsfraktion existieren allerdings noch keine Leitlinien zur Therapie, sodass diese zum größten Teil theoretisch und empirisch erfolgt. Dabei steht nicht die ursächliche Therapie der Herzinsuffizienz im Vordergrund, sondern die Behandlung bestehender Komorbiditäten und der daraus entstehenden Symptome.

So zeigt die vorliegende Studie, dass Patienten mit erhaltener Ejektionsfraktion vorrangig ACE-Hemmer bzw. AT1-Rezeptorblocker (61,1\%) und Betablocker (52\%) eingenommen haben, die mit großer Wahrscheinlichkeit der Therapie einer häufig vorliegenden Hypertonie dienten. Auch die Einnahme von Diuretika war mit 53\% recht häufig nachweisbar und diente wahrscheinlich der Linderung der Symptome beispielsweise von peripheren Ödemen und Dyspnoe bei Belastung und/oder in Ruhe, die in der Folge einer Herzinsuffizienz entstehen. Die Einnahme von Aldosteron- Antagonisten war mit 4,7\% eher eine Ausnahme.

Auch einige frühere Therapiestudien (Ahmed et al. (2006), CHARM-Preserved (Yusuf et al. 2003), PEP-CHF (Cleland et al. 2006), I-Preserve (Massie et al. 2008), SENIORS (Flather et al. 2005)) mit herzinsuffizienten Patienten und erhaltener EF belegen bei der Vormedikation eine Einnahme von ACE-Hemmern bzw. AT1-Rezeptorblockern, Beta-blockern und Diuretika.

In den epidemiologischen Studien von Vasan et al. (1999) und Smith et al. (2003) wird deutlich, dass die Einnahme von ACE-Hemmern/AT1-Rezeptorblockern im Verhältnis zu dieser Arbeit zugrunde liegenden Studie insgesamt seltener erfolgt ist, dagegen aber 
häufiger bei HFREF (Vasan et al.: 36\%, Smith et al.: 70\%) als bei HFPEF (Vasan et al.: 19\%, Smith et al.: 34\%). Auch die Einnahme von Betablockern ist insgesamt seltener als in der vorliegenden Studie nachgewiesen, erfolgt aber häufiger bei Patienten der Gruppe mit HFPEF (Vasan et al.:32\%, Smith et al.: 40\%) als bei HFREF (Vasan et al.: 11\%, Smith et al.: 34\%). Der Grund dafür könnte eine häufig vorliegende Hypertonie bei HFPEF sein. Diuretika sind im Vergleich zu ACE-Hemmern/AT1-Rezeptorblockern und Betablockern bei Vasan et al. und Smith et al insgesamt am häufigsten eingenommen worden. Allerdings zeigt sich hier auch kaum ein Unterschied zwischen HFREF (Vasan et al.: 67\%, Smith et al.: 89\%) und HFPEF (Vasan et al.: 68\%, Smith et al.: 79\%).

Es wurde häufig postuliert, dass chronisch herzinsuffiziente Patienten mit erhaltener Pumpfunktion weniger symptomatisch sind. Auch in der vorliegenden Arbeit traten bei herzinsuffizienten Patienten mit einer erhaltenen Ejektionsfraktion zwar häufiger periphere Ödeme auf, aber insgesamt waren diese Patienten weniger symptomatisch, was auch anhand einer niedrigeren NYHA-Klasse $(2,0 \pm 0,6)$ im Vergleich zu Patienten mit systolischer Herzinsuffizienz (NYHA 2,4 $\pm 0,6$ ) deutlich wurde.

Diese Beobachtung wird durch Smith et al. (2003) bestätigt, die zeigten, dass mit 32\% mehr herzinsuffiziente Patienten mit reduzierter EF an schwerer Dyspnoe leiden als Patienten mit erhaltener Ejektionsfraktion (20\%). Auch Bursi et al. (2006) kamen zu vergleichbaren Ergebnissen: Sie zeigen, dass ein größerer prozentualer Anteil mit einer NYHA-Klasse II oder III in der Gruppe der HFPEF vorlag (46\%) als in der Gruppe der HFREF (42\%). Allerdings wiesen im Vergleich zur HFPEF (42\%) aus der Gruppe der HFREF mehr Patienten die NYHA-Klasse IV auf (52\%). Insgesamt zeigt das Patientenkollektiv bei Bursi et al. allerdings eine schwerere Ausprägung der Symptomatik, verglichen mit der vorliegenden Studie. Dies könnte durch das ältere Patientenkollektiv bei Bursi et al. begründet sein, was wahrscheinlich eine größere Zahl an Komorbiditäten zur Folge hat und sich dementsprechend weiter auf die Symptomatik auswirkt.

In der CHARM-Preserved-Studie (Yusuf et al. 2003) entsprach die Symptomatik der Patienten in $61 \%$ der NYHA-Klasse II, welche auch in der SENIORS-Studie (Flather et al. 2005) mit 56,4\% und bei Ahmed et al. (2006) mit 58,1\% am häufigsten vertreten war. In der PEP-CHF-Studie (Cleland et al. 2006) wiesen 75,5\% die NYHA-Klasse I oder II auf, während bei I-Preserve (Massie et al. 2008) der größte Teil der Patienten in der NYHA-Klasse III zu finden war $(76,5 \%)$. 


\subsection{Komorbiditäten}

\subsubsection{Verteilung der Komorbiditäten bei HFREF und HFPEF}

In der vorliegenden Arbeit ist das gesamte Patientenkollektiv auf vorhandene typische Komorbiditäten untersucht worden. Insgesamt konnte man feststellen, dass diese sehr häufig vorliegen. Unter den Patienten mit systolischer Herzinsuffizienz wiesen 37,2\% typische Komorbiditäten auf, während sie bei 33\% der herzinsuffizienten Patienten mit erhaltener Ejektionsfraktion nachgewiesen werden konnte. Das heißt, dass die Komorbiditäten bei der systolischen Herzinsuffizienz zwar häufiger im Mittel lagen, aber insgesamt kamen in dieser Patientengruppe mehr Komorbiditäten vor. Dabei traten bei Patienten mit systolischer Herzinsuffizienz mehrheitlich Diabetes mellitus $(31,0 \%)$, Hyperurikämie (39,0\%), Renale Dysfunktion (34,4\%), Anämie (21,9\%), COPD (15,1\%), KHK $(46,2 \%)$ und Hyperlipidämie $(58,8 \%)$ auf. Nur Hypertonie $(78,4 \%)$ und Adipositas $(37,6 \%)$ konnten in der Gruppe der Herzinsuffizienz mit erhaltener Ejektionsfraktion signifikant häufiger nachgewiesen werden.

Die häufigsten Komorbiditäten unter den systolisch herzinsuffizienten Patienten waren Hypertonie (61,4\%), Hyperlipidämie (58,8\%) und KHK $(46,2 \%)$. Im Vergleich dazu war die Verteilung unter den Patienten der Gruppe der Herzinsuffizienz mit erhaltener Ejektionsfraktion ähnlich, da auch hier Hypertonie (78,4\%) und Hyperlipidämie (54\%) die häufigsten Komorbiditäten darstellten, jedoch Adipositas als die dritthäufigste Komorbidität mit 37,6\% nachgewiesen werden konnte. COPD ist in beiden Gruppen am seltensten nachgewiesen worden (HFREF: 15,1\%, HFPEF: 12,6\%).

Ein Vergleich dieser Zahlen mit früheren Epidemiologie- und Therapiestudien ist leider nur begrenzt möglich, da diese vor allem die häufigsten und damit typischsten Komorbiditäten untersuchten und keine der Studien ein vergleichbar breites Spektrum an Komorbiditäten erfasst hat. Somit ist dies die erste Studie, die alle die Komorbiditäten erfasst hat.

Entsprechend den Ergebnissen der hier vorliegenden Arbeit ließ sich auch bei Vasan et al. (75\%), Smith et al. (80\%) und Bursi et al. (86\%) ein Überwiegen der Hypertonie in der Gruppe der Herzinsuffizienz mit erhaltener Ejektionsfraktion verglichen mit der HFREF (Vasan et al. (1999): 71\%, Smith et al. (2003): 65\%, Bursi et al. (2006): 81\%) nachweisen. Vergleichbar mit unserer Arbeit ist auch die Häufigkeitsverteilung der KHK, die auch in den genannten epidemiologischen Studien bei Patienten mit systolischer Herzinsuffizienz (Vasan et al.: 72\%, Smith et al.: 76\%) im Gegensatz zu herzinsuffizienten Patienten mit erhaltener Pumpfunktion (Vasan et al.: 57\%, Smith et al.: 24\%) signifikant häufiger nachgewiesen werden konnte, allerdings mit einem größeren prozentualen Anteil. Das verdeutlicht, dass 
eine KHK neben dem arteriellen Hypertonus eine der häufigsten Ursachen für eine Herzinsuffizienz darstellt.

Nicht vergleichbar mit Ergebnissen unserer Studie ist die Verteilung von Diabetes mellitus. So zeigten Vasan et al. (1999) einen vorhandenen Diabetes mellitus vermehrt in HFPEF (46\%) als in HFREF (22\%), während die Verteilung bezüglich der HFREF und HFPEF bei Smith et al. (2003) vollkommen gleich war (jeweils 48\%). Nur Bursi et al. (2006) zeigten ein leichtes Überwiegen des Diabetes mellitus bei der HFREF (HFPEF: 36\%, HFREF: 38\%).

Auch das Vorliegen einer respiratorischen Erkrankung bzw. COPD ist in den epidemiologischen Studien häufiger bei HFPEF (Smith et al.: 31\%, Bursi et al.: 38\%) als bei HFREF (Smith et al.: 26\%, Bursi et al.: 30\%) gefunden worden, was ebenfalls unseren Ergebnissen widerspricht. Allerdings wurde bei Smith et al. ganz allgemein eine respiratorische Erkrankung erfasst, das heißt, es wurde nicht explizit nach einer COPD gesucht. Außerdem ist das gemeinsame Hauptsymptom der COPD und der chronischen Herzinsuffizienz die Dyspnoe. Das erschwert häufig die eindeutige Differenzierung zwischen den beiden Erkrankungen und kann somit zu Verschiebungen bezüglich der Häufigkeitsverteilung führen.

Smith et al. und Bursi et al. haben zusätzlich das Vorhandensein einer renalen Dysfunktion untersucht. Verglichen mit unserer Studie, bei der eine renale Dysfunktion signifikant häufiger bei der HFREF (34,5\%) als bei der HFPEF $(26,4 \%)$ vorkam, konnten bei deren Häufigkeitsverteilung kaum Unterschiede bezüglich HFREF oder HFPEF aufgezeigt werden.

Das Vorliegen einer Anämie ist zusätzlich von Bursi et al. untersucht worden und war etwas häufiger in der Gruppe der HFPEF zu finden, während die ebenfalls untersuchte Hyperlipidämie moderat häufiger unter den Patienten mit HFREF auftrat.

Allgemein kamen die genannten Komorbiditäten in diesen epidemiologischen Studien nicht nur insgesamt mit einem größeren prozentualen Anteil im Vergleich zur vorliegenden Arbeit vor, sondern es scheint, als sei auch eine größere Zahl an Komorbiditäten unter der Gruppe der Herzinsuffizienz mit erhaltener EF im Vergleich zur systolischen Herzinsuffizienz vertreten gewesen. Allerdings setzen sich diese drei Studien aus einem viel kleineren Kollektiv zusammen und sind dementsprechend nicht so repräsentativ bezüglich der Verteilung wie die hier vorliegende Studie.

Ein ähnliches Bild zeigte die Verteilung in Therapiestudien. In diesen wurden herzinsuffiziente Patienten mit erhaltener Ejektionsfraktion nur auf das Vorliegen einzelner Komorbiditäten wie KHK bzw. den Zustand nach Myokardinfarkt, Hypertonie und Diabetes mellitus untersucht und sind daher nur begrenzt mit den Ergebnissen dieser Arbeit zu vergleichen, für die im Gegensatz dazu eine sehr große Zahl an Komorbiditäten untersucht 
worden sind. Am häufigsten ist auch in diesen Studien ein Hypertonus gefunden worden (Ahmed et al. (2006): 59,8\%; SENIORS (Flather et al. 2005): 61,7\%; CHARM-Preserved (Yusuf et al. 2003): 64,3\%; I-Preserve (Massie et al. 2008): 88,5\%; PEP-CHF (Cleland et al. 2006): 79\%), der die Hauptsursache für die Entstehung einer Herzinsuffizienz mit erhaltener Ejektionsfraktion darstellt.

Diabetes mellitus wurde ebenfalls auf seine Häufigkeit geprüft und war mit 20,5 bis $28,8 \%$ in allen oben genannten Therapiestudien zur HFPEF ähnlich häufig vertreten und entspricht dem prozentualen Anteil von Patienten mit HFPEF und Diabetes mellitus in der hier vorliegenden Arbeit.

Die Prüfung auf eine vorhandene KHK erfolgte nur in der SENIORS-Studie und entsprach einem Anteil von 68,3\% der Patienten, was den KHK-Anteil in der Gruppe der HFPEF unserer Arbeit bei weitem übertrifft. Dies liegt wahrscheinlich an dem Mindestalter von 70 Jahren und dem höheren mittleren Alter unter den Patienten der SENIORS-Studie, was beispielweise ein höheres Risiko an Komorbiditäten zur Folge hat. Anstelle der Untersuchung auf eine vorhandene KHK erfolgte der Nachweis eines zurückliegenden Myokardinfarktes bei Ahmed et al. (49,5\%), CHARM-Preserved (44,4\%), I-Preserve (23,5\%) und PEP-CHF (26,5\%). Diese Häufigkeitsverteilung entspricht eher dem prozentualen Anteil von KHK unter der Patientengruppe mit HFPEF.

I-Preserve untersuchten zusätzlich das Vorliegen einer Anämie, die mit einem Anteil von 12,5\% ähnlich dem Vorliegen einer Anämie unter HFPEF in der vorliegenden Arbeit ist $(14,1 \%)$.

Insgesamt ist die Vergleichbarkeit mit den genannten Therapie- und Epidemiologiestudien stark eingeschränkt. Einerseits bestehen vor allem die Epidemiologiestudien aus nur einer kleinen Anzahl an Erkrankten, andererseits weisen die Studien aufgrund ihrer Ein- und Ausschlusskriterien ein spezielles Design mit definierten Patienteneigenschaften und ausgewählten Komorbiditäten auf und sind in ihrer Aussagekraft limitiert.

Der Vorteil der vorliegenden Arbeit besteht in dem viel größeren und breiterem Patientenkollektiv im Vergleich zu früheren epidemiologischen Studien und der daraus hervorgehenden großen Aussagekraft bezüglich der Verteilung von Komorbiditäten unter herzinsuffizienten Patienten und deren Ausprägung auf die Symptomatik. Außerdem wurde in unserer Studie erstmals ein breites Spektrum typischer Komorbiditäten erfasst, während in zurückliegenden Studien zur Epidemiologie und Therapie der Herzinsuffizienz hauptsächlich vereinzelt typische Risikofaktoren der Herzinsuffizienz wie Hypertonie, KHK und Diabetes mellitus beleuchtet wurden, wodurch diese immer nur begrenzt Einblick in die Zusammenhänge von Komorbiditäten und Herzinsuffizienz gaben. 


\subsubsection{Verhältnis/Einfluss pathophysiologischer Kenngrößen und Komorbiditäten auf die Symptomatik}

Unsere Studie untersuchte bei HFREF und HFPEF den Einfluss einzelner Komorbiditäten auf die Ausprägung der Symptomatik anhand der NYHA-Klasse, adjustiert auf wichtige demographische Faktoren wie Alter und Geschlecht.

Da Komorbiditäten selten solitär auftreten, wurde der Einfluss der Komorbiditäten auch bei Anwesenheit anderer Begleiterkrankungen mittels multivariater Analyse überprüft. Das Ausmaß der Einflussgrößen wurde mit dem Ausmaß des Einflusses von linksventrikulärer Funktion und linksventrikulärem Remodeling verglichen.

\subsubsection{Gleichgerichtete Interaktionen von Komorbiditäten und Symptomatik bei HFREF und HFPEF}

Die Komorbiditäten Diabetes mellitus, Hyperurikämie, Anämie und renale Dysfunktion wiesen einen vergleichbaren Einfluss auf die NYHA- Klasse bei chronischer Herzinsuffizienz mit reduzierter und erhaltener Ejektionsfraktion auf. Es bestand jeweils eine signifikante Assoziation mit einer höheren NYHA-Klasse bei Vorliegen einer der genannten Komorbiditäten.

\section{Anämie}

Eine Anämie bei chronisch herzinsuffizienten Patienten, die ihm Rahmen chronischer Erkrankungen, bei relativem EPO-Mangel, Plasmavolumenüberladung oder exzessiver Zytokinproduktion etc. entstehen kann, wurde bei 15 bis 30\% dieser Patienten nachgewiesen und stellt somit eine äußerst häufige und relevante Komorbidität dar. Die Auswirkungen einer Anämie wurden bisher in einigen Studien zur chronischen, systolischen Herzinsuffizienz untersucht und sind mittlerweile bekannt: Eine vorhandene Anämie bei chronischer Herzinsuffizienz ist assoziiert mit einer höheren Mortalität, reduzierter linksventrikulärer Ejektionsfraktion, niedrigem kardialen funktionalen Status, höherer Hospitalisierungsrate, Zeichen von Mangelernährung, eingeschränkter körperlicher Belastbarkeit und Leistungsfähigkeit, einer progressiven Verschlechterung der renalen Funktion, steigendem Bedarf an Diuretika, Hyponaträmie, erhöhtem Plasmavolumen, reduziertem Erythrozytenvolumen und einer eingeschränkten Lebensqualität (Wexler et al. 2005).

In welchem Maß sich eine Anämie tatsächlich auf die Symptomatik, Lebensqualität und Morbidität der Patienten auswirkt und welche positiven Ergebnisse eine Korrektur des Hämoglobins nach sich zieht, wurde in einigen früheren Studien zur systolischen Herzinsuffizienz bereits gezeigt. So konnte entsprechend den Ergebnissen von Mancini et al. (2003) und Kalra et al. (2003), die mittels der Bestimmung der $\mathrm{mVO}_{2}$ - Verwertung unter 
höchster Belastung die Herzfunktion und die Belastbarkeit überprüft haben, bei systolisch herzinsuffizienten Patienten ein signifikanter Abfall des peak $\mathrm{mVO}_{2}$ mit absinkendem Hämoglobin gezeigt werden.

Unter der Annahme, dass eine Normalisierung der Hämoglobinkonzentration in chronisch herzinsuffizienten Patienten eine Verbesserung der körperlichen Leistungsfähigkeit durch eine verbesserte Sauerstoffzufuhr nach sich ziehen könnte bzw. alternativ ein steigendes Hämoglobin oxidativen Stress reduzieren und damit die vasodilatatorische Kapazität und/oder die Sauerstoffzufuhr verbessern könnte, wurden bisher kontrollierte und nicht kontrollierte Studien durchgeführt, bei denen eine Korrektur der Anämie mittels subkutanem Erythropoetin, teilweise in Addition zu intravenösem Eisen, erfolgte und die eine Verbesserung in den oben genannten Parametern wie der kardialen Funktion und Hospitalisierungsrate zeigte (Jin et al. 2010). Allerdings wurden diese Studien ausschließlich für die systolische Herzinsuffizienz durchgeführt.

Mancini et al. (2003) behandelten in einer placebokontrollierten Studie schwer chronisch herzinsuffiziente Patienten mit eingeschränkter Pumpfunktion mit Erythropoetin über drei Monate. Dabei zeigten sie eine signifikante Verbesserung des peak $\mathrm{VO}_{2}$, der $\mathrm{VO}_{2}$ an der anaeroben Schwelle, der Belastbarkeitsdauer und der Distanz im 6-Minuten-Gehtest. In der Kontrollgruppe konnten keine signifikanten Veränderungen beobachtet werden.

Ebenso zeigten Kalra et al. (2003) bei einem Anheben der Hämoglobinkonzentration und der daraus resultierenden Änderung des peak $\mathrm{VO}_{2}$ eine signifikant positiv lineare Ausbesserung. Und auch Silverberg et al. (2001) bewiesen eine Verbesserung der NYHA-Klasse bei Patienten, die Epoetin alfa erhielten, im Vergleich zur Kontrollgruppe. Damit werden die Ergebnisse dieser hier vorliegenden Studie bezüglich des Zusammenhangs für eine HFREF eindrücklich bestätigt.

Während sich all diese erhobenen Ergebnisse nur auf chronisch herzinsuffiziente Patienten mit eingeschränkter Ejektionsfraktion bezogen haben, so sind die Konsequenzen einer Anämie bei Herzinsuffizienz mit erhaltener Pumpfunktion unzureichend untersucht worden und der Stellenwert dieser Komorbidität weitestgehend unklar. Die hier vorliegende Studie ist die erste Studie, in der die Einflüsse einer Anämie auf herzinsuffiziente Patienten mit erhaltener Ejektionsfraktion untersucht worden sind und die in einem solch großen repräsentativen Patientenkollektiv durchgeführt worden ist. In unserer Studie konnte gezeigt werden, dass eine Anämie unabhängig vom Geschlecht auch bei der HFPEF einen signifikant negativen Einfluss auf die Symptomatik hat. Das heißt, eine begleitende Anämie ging dementsprechend nicht nur bei den systolisch herzinsuffizienten Patienten, sondern auch bei den herzinsuffizienten Patienten mit erhaltener Pumpfunktion mit einer höheren NYHA-Klasse einher. 
Frühere Studien und vor allem die zu der hier vorliegenden Arbeit zeigen, dass eine gleichzeitig vorhandene Anämie noch zur Verschlechterung der bereits bestehenden Symptomatik chronischer Herzinsuffizienz beiträgt. Es muss also diskutiert werden, gerade weil die Behandlung der HFPEF noch nicht etabliert ist, ob eine Korrektur der Anämie bei den Patienten sinnvoll ist. Dies hätte möglicherweise eine Verbesserung der Symptomatik sowie der körperlichen Belastbarkeit und Leistungsfähigkeit zur Folge und könnte eine Verlangsamung oder einen Stillstand der Progression der Herzinsuffizienz bewirken. Dies muss aber in zukünftigen Studien untersucht werden.

\section{Renale Dysfunktion}

Es ist bereits bekannt, dass eine reduzierte glomeruläre Filtrationsrate bei chronisch herzinsuffizienten Patienten zu einer Einschränkung der intrakardialen Erregungsleitung und der diastolischen Funktion führt (Bruch et al. 2007). Ebenso haben Hillege et al. (2000) herausgefunden, dass die renale Dysfunktion eine Einflussvariable bezüglich der Mortalität bei stabilen Patienten mit fortgeschrittener Herzinsuffizienz darstellt. Dabei kann auch eine unkontrollierte chronische Herzinsuffizienz zu einem weiteren Abfall der glomerulären Filtrationsrate bei renaler Dysfunktion führen.

Des Weiteren ist eine renale Dysfunktion ein Faktor, der zur Entstehung einer Anämie in herzinsuffizienten Patienten beiträgt. Die Anämie wiederum fördert die Entstehung einer linksventrikulären Hypertrophie. Jede dieser drei Konditionen kann zur Exazerbation der anderen führen. Man spricht deshalb auch von einem Kardio-Renalen-Anämie-Syndrom. Zur Durchbrechung dieses Circulus vitiosus spielt die Korrektur der Anämie eine wichtige Rolle, was zu einer Verbesserung der kardialen Funktion und körperlichen Leistungsfähigkeit sowie einer Stabilisierung der renalen Funktion und Verbesserung der Lebensqualität führen kann (Silverberg et al. 2004).

Um den Stellenwert der Komorbidität „renale Dysfunktion“ als potentiellen Angriffspunkt für Therapieoptionen der chronischen Herzinsuffizienz zu beurteilen, sind Ergebnisse über den Einfluss einer eingeschränkten renalen Funktion auf die Belastbarkeit und das klinische Bild der Patienten vonnöten.

Sietsema et al. (2004) konnten durch die Bestimmung der Sauerstoffaufnahme unter maximaler Belastung (peak $\mathrm{VO}_{2}$ ) zeigen, dass Patienten mit einer fortgeschrittenen Nierenerkrankung im Vergleich zu Gesunden eine reduzierte körperliche Belastbarkeit aufwiesen. Man kann also vermuten, dass eine zusätzliche renale Dysfunktion bei herzinsuffizienten Patienten zu einer schwerwiegenderen Symptomatik führt, aber Studienergebnisse existieren zu dieser Annahme bisher noch nicht. 
Die hier vorliegende Arbeit ist die erste, in der die Konsequenzen einer eingeschränkten renalen Funktion bezüglich der Symptomatik in einem großen Kollektiv herzinsuffizienter Patienten untersucht wurden. Es konnte nachgewiesen werden, dass eine renale Dysfunktion einen signifikant negativen Einfluss auf die NYHA-Klasse hat. Eine eingeschränkte Nierenfunktion geht dementsprechend mit einer ausgeprägteren Herzinsuffizienzsymptomatik einher. Ein Unterschied wurde weder für das Vorliegen einer eingeschränkten oder erhaltenen Ejektionsfraktion noch bezüglich des Geschlechts erhoben. Anhand dieser Studie, in der erstmalig die Beziehung zwischen renaler Dysfunktion und NYHA-Klasse untersucht wurde, kann die Vermutung abgeleitet werden, dass eine Therapie und Kontrolle einer eingeschränkten Nierenfunktion den Progress einer Herzinsuffizienz aufhalten können und einen positiven Effekt auf den klinischen Status und die Belastbarkeit herzinsuffizienter Patienten mit eingeschränkter sowie erhaltener Ejektionsfraktion haben. Der klare Zusammenhang muss jedoch in zukünftigen Studien untersucht werden.

\section{Diabetes mellitus}

Diabetes mellitus ist bekannterweise ein unabhängiger Risikofaktor für die Entstehung der Herzinsuffizienz, dessen Prävalenz 20 bis $25 \%$ bei chronisch herzinsuffizienten Patienten beträgt. Diabetes kann ebenfalls zur Progression der Herzinsuffizienz durch eine beschleunigte Atherosklerose beitragen und ebenso zu einer exzessiven Akkumulation interstitiellen myokardialen Kollagens führen, woraus eine weitere Verschlechterung der systolischen und diastolischen Funktion resultiert.

Ergebnisse über die Auswirkungen eines gleichzeitig vorhandenen Diabetes mellitus auf das klinische Bild, die Lebensqualität und die Belastung herzinsuffizienter Patienten wurden bislang nur für die systolische Herzinsuffizienz erhoben. So wiesen die Diabetiker in einer Substudie von RESOLVD eine reduzierte funktionelle Kapazität und eine deutlich stärkere Herzinsuffizienzsymptomatik auf (Suskin et al. 2000). Andere Studien demonstrierten bei Diabetikern mit chronisch systolischer Herzinsuffizienz eine Beeinträchtigung im 6-MinutenGehtest (Ingle et al. 2006), reduzierte körperliche Belastbarkeit (Tibb et al. 2005) und herabgesetzte pulmonale Funktion im Vergleich zu Nicht-Diabetikern (Guazzi et al., 2002). Ferner haben Tibb et al. (2005) gezeigt, dass Patienten mit Diabetes mellitus und linksventrikulärer systolischer Dysfunktion (LVSD) verglichen mit Patienten mit einer LVSD ohne Diabetes eine niedrigere Sauerstoffaufnahme bei maximaler Belastung (peak $\mathrm{VO}_{2}$ ) aufwiesen. Des Weiteren stellten Ingle et al. (2006) fest, dass Diabetiker mit LVSD, im Vergleich zu Nicht-Diabetikern mit LVSD, eine schlechtere Leistung im 6-Minuten-Gehtest erbrachten. 
Aber nicht nur Diabetiker, sondern auch Nicht-Diabetiker mit einer Insulinresistenz oder Hyperinsulinämie wiesen eine erweiterte und schwerere Herzinsuffizienzsymptomatik und eine kürzere 6-Minuten-Gehtest-Distanz im Vergleich zu Nicht-Diabetikern ohne Insulinresistenz auf (Suskin et al. 2000).

Wie sich aber im Gegensatz zur systolischen Herzinsuffizienz das Vorliegen eines Diabetes mellitus auf die Belastbarkeit chronisch herzinsuffizienter Patienten mit erhaltener Ejektionsfraktion auswirkt, ist kaum erforscht und es existieren nur wenige Daten. Fang et al. (2005) haben bei Patienten mit einem Typ-2-Diabetes und einer EF $>50 \%$ festgestellt, dass diese eine eingeschränkte kardiale Leistung infolge der steigenden Arbeitslast des Herzens aufweisen, die mit einer linksventrikulären diastolischen Dysfunktion einhergeht. Außerdem fanden sie heraus, dass ein relativ begrenztes Schlagvolumen beim Typ-2-Diabetes die kardiale Auswurfleistung unter maximaler Belastung hemmt und so die körperliche Leistungsfähigkeit der Patienten limitiert.

Die hier vorliegende Arbeit ist die erste Studie, die sich mit den Konsequenzen eines vorhandenen Diabetes mellitus bezüglich der Symptomatik chronisch herzinsuffizienter Patienten mit erhaltener EF beschäftigt.

Welche Relevanz diese Komorbidität hat, wird anhand unserer erhobenen Daten deutlich, denn immerhin wiesen $24,2 \%$ der herzinsuffizienten Patienten mit erhaltener Pumpfunktion einen Diabetes mellitus auf. Es konnte gezeigt werden, dass ein gleichzeitig bestehender Diabetes mellitus nicht nur bei chronisch herzinsuffizienten Patienten mit reduzierter Pumpfunktion, sondern eben auch bei Patienten mit erhaltener Ejektionsfraktion mit einer schlechteren NYHA-Klasse einherging und dementsprechend in beiden Patientengruppen einen signifikant negativen Einfluss auf die Symptomatik ausübte.

Es existiert keine vergleichbare Studie mit einem Patientenkollektiv in der Größe der hier vorliegenden Arbeit. Diese Ergebnisse sind daher sehr repräsentativ und verdeutlichen die Relevanz, einen bestehenden Diabetes mellitus optimal zu therapieren, um somit eine weitere Beeinträchtigung des klinischen Bildes chronisch herzinsuffizienter Patienten zu verhindern. Dementsprechend sind für HFPEF sowie für HFREF weitere Studien erforderlich, um Interventionen zu ermitteln, die die Korrektur einer Insulinresistenz ermöglichen, um auch auf diesem Weg eine Verschlechterung der Symptomatik der chronischen Herzinsuffizienz zu vermeiden.

Einen möglichen Angriffspunkt könnte die Studie von Guazzi et al. (2003) bieten. Die Haupterkenntnis dieser Studie war, dass die Gabe von Insulin bei Patienten mit systolischer Herzinsuffizienz und Diabetes mellitus Typ 2 eine Verbesserung der $\mathrm{VO}_{2}$ und der Ventilation unter Belastung zur Folge hatte. Außerdem war die Insulingabe assoziiert mit einer 
Verbesserung von $\mathrm{VE} / \mathrm{VCO}_{2}$ und einem steigenden peak $\mathrm{VO}_{2}$ und führte somit zu einer Optimierung der physikalischen Leistungsfähigkeit der Patienten.

\section{Hyperurikämie}

Eine Hyperurikämie bzw. ein erhöhter Serum-Harnsäurespiegel stellt bekannterweise einen unabhängigen Risikofaktor für chronische Niereninsuffizienz, kardiovaskuläre Erkrankungen und Hypertonie dar. Darüber hinaus prognostiziert eine Hyperurikämie ein schlechteres Überleben bei schwerer chronischer Herzinsuffizienz. Insgesamt ist der Einfluss einer bestehenden Hyperurikämie bei herzinsuffizienten Patienten allerdings nur wenig untersucht.

Einige kleine Studien beschäftigten sich mit der Beziehung zwischen erhöhten Harnsäurespiegeln und der Symptomatik und Belastbarkeit bei Patienten mit systolischer Herzinsuffizienz.

So haben Leyva et al. (1998) durch die Bestimmung der anaeroben Schwelle und der Messung der Serum-Harnsäurewerte herausgefunden, dass Patienten mit chronischer Herzinsuffizienz im Vergleich zur gesunden Kontrollgruppe eine niedrigere anaerobe Schwelle und höhere Serum-Harnsäurewerte aufwiesen. Bei den chronisch herzinsuffizienten Patienten bestand eine inverse Korrelation zwischen der anaeroben Schwelle und den Harnsäurewerten in Ruhe, was dem Wissen entspricht, dass eine Verbindung zwischen der Harnsäureproduktion und der Imbalance des aeroben/anaeroben Metabolismus bei chronischer Herzinsuffizienz besteht.

Jankowska et al. (2007) haben Patienten mit stabiler, milder bis moderater Herzinsuffizienz und eingeschränkter Ejektionsfraktion untersucht. Sie zeigten, dass die SerumHarnsäurewerte parallel zur Schwere der Herzinsuffizienz, die anhand der NYHA-Klasse festgelegt wurde, anstiegen. Außerdem bestanden inverse Korrelationen zwischen den Harnsäurewerten und der peak $\mathrm{VO}_{2}$ sowie der LVEF, nicht aber mit der renalen Funktion. Jankowska et al. kamen daher zu dem Ergebnis, dass eine bestehende Hyperurikämie bei Patienten mit milder bis moderater chronischer systolischer Herzinsuffizienz eine Einschränkung der Belastbarkeit und eine Aktivierung von Entzündungsreaktionen zur Folge hat und mit einer schlechteren Prognose einhergeht.

Über den Einfluss einer Hyperurikämie auf chronische Herzinsuffizienz mit erhaltener Ejektionsfraktion existieren bisher keine Daten. Wir haben in der hier vorliegenden Studie den Einfluss einer bestehenden Hyperurikämie auf die Symptomatik bei chronischer Herzinsuffizienz untersucht und diese erstmalig zwischen reduzierter und erhaltener Ejektionsfraktion verglichen. Es konnte gezeigt werden, dass erhöhte Harnsäurewerte mit einer schlechteren NYHA-Klasse einhergehen und sich dementsprechend negativ auf die 
Symptomatik auswirken. Dieser Zusammenhang besteht außerdem unabhängig von Alter und Geschlecht.

Dementsprechend sollten erhöhte Serum-Harnsäurewerte als neues therapeutisches Angriffsziel in der chronischen Herzinsuffizienz mit eingeschränkter sowie erhaltener EF in Betracht gezogen werden und Ziel von künftigen Studien sein.

\subsubsection{Konträre Interaktionen von Komorbiditäten und Symptomatik bei HFREF und HFPEF}

Konträre Interaktionen bestanden bei der Prüfung des Einflusses von COPD, KHK und Adipositas. COPD hatte je nach Geschlecht einen unterschiedlichen Effekt auf die Symptome, während KHK und Adipositas nur bei Patienten mit erhaltener Ejektionsfraktion mit einer höheren NYHA-Klasse assoziiert waren, nicht jedoch bei Patienten mit systolischer Herzinsuffizienz.

\section{COPD}

COPD stellt mit einer Prävalenz von 20 bis $30 \%$ bei chronisch herzinsuffizienten Patienten eine häufig anzutreffende Komorbidität dar (Angermann 2009).

In der hier vorliegenden Studie kommt eine COPD bei 15,1\% der systolisch herzinsuffizienten Patienten und bei $12,6 \%$ der herzinsuffizienten Patienten mit erhaltener Ejektionsfraktion vor. Die Häufigkeit einer hier vorliegenden COPD ist im Vergleich zu anderen Komorbiditäten seltener. Das könnte dadurch erklärt werden, dass die Existenz einer COPD häufig durch die Klinik der Herzinsuffizienz maskiert wird, da beide Erkrankungen als Hauptsymptom eine Dyspnoe aufweisen. COPD führt zu einer reduzierten pulmonalen Funktion, zu Dyspnoe und Einschränkungen der Belastbarkeit, während eine chronische Herzinsuffizienz ähnliche Limitationen aufweist, nämlich Dyspnoe, Erschöpfung und Reduktion der körperlichen Leistungsfähigkeit (Karapolat et al. 2008).

Es ist bekannt, dass COPD-Patienten mit einer bestehenden Herzinsuffizienz im Vergleich zu COPD-Patienten ohne Herzinsuffizienz eine signifikant höhere Mortalität und Hospitalisierungsrate aufweisen (Boudestein et al. 2009). Ebenfalls existieren Studien, in denen jeweils der Effekt der Herzinsuffizienz und der COPD auf die funktionelle Kapazität, Leistungsfähigkeit und Lebensqualität untersucht wurde. Aber es existieren keine Daten, in denen der Einfluss einer Koexistenz beider Erkrankungen auf den klinischen Status und die Symptomatik erhoben wurde.

So untersuchten Karapolat et al. (2008) eine Patientengruppe mit COPD im Vergleich zu einer Patientengruppe mit systolischer Herzinsuffizienz bezüglich der Symptomatik (BorgSkala), Lebensqualtität (SF36) und funktionalen Kapazität (kadiopulmonaler Belastungstest). 
Während in einer Studie von Caroci Ade und Lareau (2004) von einer stärkeren DyspnoeSymptomatik bei COPD-Patienten im Gegensatz zu herzinsuffizienten Patienten berichtet wurde, bestand bei Karapolat et al. kein signifikanter Unterschied zwischen den beiden Gruppen bezüglich der Borg-Dyspnoe-Score oder der Lebensqualität. Ein signifikanter Zusammenhang zwischen den objektiven Erkrankungsindikatoren (FEV1 und LVEF) konnte in beiden Gruppen weder mit der Borg-Dyspnoe-Score noch mit der Lebensqualität oder der funktionellen Kapazität $\left(\mathrm{VO}_{2}\right)$ gefunden werden. Aber eine signifikant negative Korrelation bestand in beiden Patientengruppen zwischen Borg-Score und dem funktionellen Kapazitätstest.

Terziyski et al (2009) untersuchten die funktionelle Kapazität bei herzinsuffizienten Patienten und COPD-Patienten. Sie ermittelten den Anstieg der Sauerstoffaufnahme mithilfe eines Belastungstests und kamen $\mathrm{zu}$ dem Ergebnis, dass beide Patientengruppen in der Gegenüberstellung zur gesunden Kontrollgruppe einen vergleichsweise signifikanten Abfall der körperlichen Leistungsfähigkeit aufwiesen.

Aufgrund dieser vorherigen Studien, welche beweisen, dass jede Krankheit für sich eine erhebliche negative Auswirkung auf das klinische Bild der Patienten hat, kann man annehmen, dass eine Koexistenz einer chronischen Herzinsuffizienz und einer COPD noch eine schwerere und ausgeprägtere Symptomatik zu Folge hat.

In der hier vorliegenden Studie wurde erstmalig der Einfluss einer bestehenden COPD auf die Ausprägung der Herzinsuffizienzsymptomatik analysiert. Ebenfalls ist es die erste Studie mit einem so großen Patientenkollektiv zu diesem Thema. Eine vorhandene COPD zeigte je nach Geschlecht unterschiedliche Effekte auf die Symptomatik herzinsuffizienter Patienten. Während eine COPD beim männlichen Geschlecht mit einer schlechteren NYHA-Klasse und damit einer schwerwiegenderen Symptomatik einherging, hatte eine vorliegende COPD unter Frauen keinen signifikanten Einfluss auf die NYHA-Klasse. Ebenfalls wurde die Interaktion mit der Ejektionsfraktion untersucht. Aber es konnte kein signifikanter Unterschied bezüglich eines Effektes der COPD auf Herzinsuffizienz mit reduzierter und erhaltener Ejektionsfraktion festgestellt werden.

Eine mögliche Ursache bezüglich der unterschiedlichen Auswirkung auf die Herzinsuffizienzsymptomatik in Abhängigkeit vom Geschlecht könnte sein, dass Männer, im Gegensatz zu Frauen, möglicherweise einen höheren Zigarettenkonsum und damit mehr „pack years“ aufweisen. Deshalb haben Männer wahrscheinlich eine schwerere COPD, was die Korrelation mit einer schlechteren NYHA-Klasse in unserer Studie erklären würde. In der hier vorliegenden Arbeit wurde allerdings weder der Schweregrad einer COPD noch eine bestehende Vormedikation erfasst. Daher sind weitere Studien notwendig, um dieses Phänomen zu klären. 
Prinzipiell sollte besonders beim männlichen Geschlecht versucht werden, die Diagnose einer COPD neben einer Herzinsuffizienz zu stellen und diese neben der Behandlung der Herzinsuffizienz als therapeutisches Ziel streng zu verfolgen, unabhängig davon, ob es sich um eine reduzierte oder erhaltene Pumpfunktion handelt.

\section{KHK}

Die KHK ist bekannterweise ein bedeutender Risikofaktor für die Entstehung und auch die Progression der Herzinsuffizienz. Obwohl eine Herzinsuffizienz infolge einer koronaren Herzerkrankung ein verbreitetes Problem mit einer schlechteren Prognose darstellt (Teerlink et al. 1991) und die Mortalität in einem solchen Fall signifikant höher ist als bei einer Herzinsuffizienz mit nicht-ischämischer Ätiologie (Likoff et al. 1987), ist der Einfluss der KHK auf die Symptomatik, den klinischen Status und die Leistungsfähigkeit herzinsuffizienter Patienten nur wenig untersucht.

Bisher existieren nur Studien, die sich mit der Belastbarkeit koronar herzkranker Patienten vor und nach kardialen Eingriffen beschäftigt haben und daher nur begrenzt mit der vorliegenden Arbeit vergleichbar sind.

Townend et al. (1995) haben den Effekt einer operativen Revaskularisierung auf den klinischen Status und die Belastungskapazität koronar herzkranker Patienten mit einer stark eingeschränkten ventrikulären Funktion, die eine symptomatische Herzinsuffizienz ohne Angina pectoris erzeugt, untersucht. Drei Monate nach dem Eingriff konnte ein signifikanter Anstieg in der Belastungszeit und der peak $\mathrm{VO}_{2}$ nachgewiesen werden. Es konnte auch eine Verbesserung der NYHA-Klasse einiger Patienten beobachtet werden. Die LVEF in Ruhe änderte sich nicht, aber insgesamt führte eine Revaskularisierung zu einer Verbesserung der Symptome und der Belastungstoleranz.

Fiorina et al. (2007) führten vor und 15 Tage nach verschiedenen kardialen Operationen (65\% Bypass-OP, 25\% Herzklappenersatz, 4\% beides, 4\% andere OPs) einen 6-MinutenGehtest durch. Unter anderem fanden sie heraus, dass Patienten nach einer Bypass-OP im Vergleich zu Patienten ohne Bypass-OP, eine signifikant längere Strecke im 6-MinutenGehtest zurücklegten und dementsprechend eine bessere Belastbarkeit aufwiesen.

In einer weiteren Studie (Erentürk et al. 2007) wurde ebenfalls das Ergebnis einer Revaskularisation bei Patienten mit schwerer Einschränkung der linksventrikulären Ejektionsfraktion bei ischämischer Herzinsuffizienz untersucht. Auch hier hatte eine Bypassoperation der Koronararterien neben einer Verbesserung der LVEF einen positiven Einfluss auf die NYHA-Klasse und damit eine Verbesserung der Symptomatik zur Folge.

Nieuwland et al. (1998) analysierten die Belastbarkeit einer Patientengruppe mit milder linksventrikulärer Dysfunktion (EF= 35 bis 55\%) und altem Myokardinfarkt (>4 Wochen) und 
verglichen diese Ergebnisse mit einer Kontrollgruppe, die ebenfalls einen alten Myokardinfarkt, aber eine normale LVEF (>55\%) aufwies. Der mittlere peak $\mathrm{VO}_{2}$ war bei den Patienten mit milder linksventrikulärer Dysfunktion niedriger: bei 75\% der Patienten war der peak $\mathrm{VO}_{2}$ gesunken und bei $18 \%$ war er stark gesunken. In der Kontrollgruppe zeigten nur $18 \%$ einen Abfall des peak $\mathrm{VO}_{2}$. Hier wurde die Leistungsfähigkeit also eher durch eine Reduktion der LVEF als durch eine gleichzeitig bestehende KHK beeinträchtigt.

In all diesen Studien wurde folglich gezeigt, dass eine operative Sanierung einer KHK mit einer Verbesserung der Leistungsfähigkeit einhergeht. Dennoch wurde der Einfluss der KHK als Komorbidität einer bestehenden chronischen Herzinsuffizienz vor allem bezüglich der körperlichen Leistungsfähigkeit und Symptomatik bisher nicht untersucht. Wir haben den Einfluss einer gleichzeitig bestehenden KHK auf die Ausprägung der Symptomatik herzinsuffizienter Patienten mit reduzierter im Vergleich zur erhaltenen Ejektionsfraktion untersucht und miteinander verglichen. Während frühere Studien meist aus kleinen Probandengruppen bestanden, häufig nur das männliche Geschlecht untersuchten und auf Ergebnisse kardialer Eingriffe angewiesen waren, beschäftigte sich die hier vorliegende Studie mit einem großen Patientenkollektiv und untersuchte darüber hinaus auch die Interaktionen mit deren Geschlecht und Alter.

Die KHK stellte in dieser Studie eine sehr häufige und damit relevante Komorbidität dar. Unter systolisch herzinsuffizienten Patienten kam sie mit 46,2\% signifikant häufiger vor und hatte bei herzinsuffizienten Patienten mit erhaltener Ejektionsfraktion einen Anteil von 31,3\%. Es konnte beobachtet werden, dass eine vorhandene KHK in beiden Patientengruppen mit einer vermehrten Ausprägung der Dyspnoe einherging. Ein signifikant negativer Effekt auf die NYHA-Klasse beruhte aber maßgeblich auf der Assoziation mit HFPEF und weniger mit HFREF. Eine Interaktion bezüglich des Geschlechts konnte nicht gezeigt werden.

In dieser Studie erfolgte keine weitere Analyse bzw. Beurteilung der Schwere der vorliegenden KHK. Eine mögliche Erklärung für den unterschiedlichen Einfluss auf die NYHA-Klasse könnte daher sein, dass Patienten mit HFPEF im Vergleich zu systolisch herzinsuffizienten Patienten möglicherweise eine ausgeprägtere oder nicht effizient behandelbare KHK aufwiesen und dementsprechend durch eine schwerere Ausprägung der Symptomatik gekennzeichnet waren.

Da eine gleichzeitig bestehende KHK in der Gruppe HFREF sowie der HFPEF mit einer Verschlechterung der Symptomatik assoziiert war - wenn auch nur unter HFPEF-Patienten signifikant -, sollte die KHK in Anbetracht ihrer Häufigkeit als therapeutisches Angriffsziel zur Verbesserung des klinischen Status chronisch herzinsuffizienter Patienten genutzt werden. 


\section{Adipositas}

Adipositas ist als Risikofaktor für die Entstehung kardiovaskulärer Erkrankungen, die Herzinsuffizienz eingeschlossen, bekannt. Schätzungsweise 20 bis $30 \%$ der herzinsuffizienten Patienten sind übergewichtig (Horwich et al. 2009). Dennoch ist der Stellenwert von Adipositas bei herzinsuffizienten Patienten bisher noch unzureichend untersucht.

Man weiß, dass übergewichtige Patienten verstärkt Dyspnoe und Einschränkungen in der körperlichen Belastbarkeit aufweisen, was wiederum die Lebensqualität entscheidend beeinflusst. Die hierfür hauptsächlich verantwortlichen respiratorischen Ursachen sind ein erhöhter Ventilationsbedarf, vermehrte Atemarbeit, respiratorische Muskelinsuffizienz und eine verminderte respiratorische Compliance (Parameswaran et al. 2006).

Gallagher et al. (2005) haben die kardiovaskuläre Fitness jeweils in einer Gruppe systolisch herzinsuffizienter Patienten und einer Gruppe morbid Adipöser (BMl>40 kg/m², bzw. BMl>35 $\mathrm{kg} / \mathrm{m}^{2}$ bei vorliegendem Diabetes mellitus) untersucht und mit einer Kontrollgruppe verglichen. Sie kamen zu dem Ergebnis, dass die maximale Sauerstoffaufnahme $\left(\mathrm{VO}_{2}\right.$ max $)$ adipöser Patienten ähnlich der von systolisch herzinsuffizienten Patienten war, aber insgesamt niedriger als in der Kontrollgruppe. In der Kontrollgruppe konnte allerdings ein inverser Zusammenhang zwischen $\mathrm{BMI}$ und $\mathrm{VO}_{2}$ max beobachtet werden. Diese Ergebnisse suggerieren also, dass eine Einschränkung der $\mathrm{VO}_{2}$ max morbid adipöser Patienten mit dem BMI in Beziehung steht.

In einer Studie zur Reproduzierbarkeit des 6-Minuten-Gehtests bei adipösen Erwachsenen ist gezeigt worden, dass eine negative Korrelation zwischen der zurückgelegten Gehstrecke und dem BMI zum einen und dem Taillenumfang zum anderen, besteht (Beriault et al. 2009). Vor kurzem wurde der Zusammenhang zwischen dem BMI und einem kardiopulmonalen Belastungstest chronisch systolisch herzinsuffizienter Patienten von Horwich et al. (2009) untersucht. Sie fanden heraus, dass ein steigender BMI invers mit der $\mathrm{VE} / \mathrm{VCO}_{2}$ korrelierte und dass bei adipösen Patienten im Vergleich zu normalgewichtigen ein Abfall des peak $\mathrm{VO}_{2}$ zu beobachten war. In der multivariaten Analyse stellten Horwich et al. fest, dass der BMI einen unabhängigen Einflusswert für die peak $\mathrm{VO}_{2}$ und die $\mathrm{VE} / \mathrm{VCO}_{2}$ bei chronisch systolischer Herzinsuffizienz darstellt.

Horwich et al. untersuchten aber nur den Einfluss von Adipositas bei systolischer Herzinsuffizienz. In unserer Studie wurde erstmalig zusätzlich der Einfluss auf die Symptomatik chronisch herzinsuffizienter Patienten mit erhaltener Ejektionsfraktion untersucht. Denn im Gegensatz zur systolischen Herzinsuffizienz, bei der Adipositas in 
26,8\% der Fälle auftrat, liegt eine Adipositas bei HFPEF mit einem Anteil von 37,6\% signifikant häufiger vor und ist daher nicht zu vernachlässigen.

In dieser Studie haben wir herausgefunden, dass eine Adipositas nur die Symptomatik chronisch herzinsuffizienter Patienten mit erhaltener Ejektionsfraktion signifikant beeinflusst und mit einer höheren NYHA-Klasse einhergeht. Und obwohl Horwich et al. eine Einschränkung der körperlichen Leistungsfähigkeit (anhand peak $\mathrm{VO}_{2}$ und $\mathrm{VE} / \mathrm{VCO}_{2}$ ) nachgewiesen haben, konnte in der hier vorliegenden Arbeit kein signifikanter Einfluss auf die Ausprägung der Herzinsuffizienzsymptomatik bei Patienten mit eingeschränkter Pumpfunktion festgestellt werden. Ebenfalls bestanden keine signifikanten Interaktionen bezüglich des Geschlechts.

Eine ausbleibende Verschlechterung der Symptomatik systolisch herzinsuffizienter Patienten könnte durch das Phänomen der „obesity paradox“ erklärt werden. Denn wie bekannt führt die Adipositas zu LV-Abnormalitäten wie exzentrischer und konzentrischer Hypertrophie, die mit einer steigenden Mortalität assoziiert sind (Lavie et al. 2009). Ebenfalls steigert eine Adipositas das Herzinsuffizienzrisiko und begünstigt die Progression einer Herzinsuffizienz (Alpert et al. 1997). Allerdings wird als „reverse Epidemiologie“ bzw. „obesity paradox“ beschrieben, dass adipöse Patienten bei bestehender Diagnose der chronischen Herzinsuffizienz ein geringeres Sterblichkeitsrisiko haben (Lavie et al. 2009). In der CHARMStudie hatte die Patientengruppe mit einem BMI von $30-33 \mathrm{~kg} / \mathrm{m}^{2}$ die niedrigste Mortalität, ebenso wie in der DIG-Studie. Die Patienten mit einem „normalen“ BMI nach WHO-Kriterien $(18,5-24,9)$ hatten im Vergleich dazu ein höheres Mortalitätsrisiko. Mögliche Erklärungen hierfür sind, dass die chronische Herzinsuffizienz einen progressiven katabolen Zustand darstellt und die adipösen herzinsuffizienten Patienten mehr metabolische Reserven aufweisen (Lavie et al. 2009). Zusätzlich weisen adipöse Patienten niedrigere Spiegel des zirkulierenden natriuretischen Peptids auf (Mehra et al. 2004) und zeigen eine abgeschwächte Aktivierung des sympathischen Nervensystems und des Renin-AngiotensinSystems (Oreopoulos et al. 2008). Ebenso führt eine vorliegende Adipositas zu einer Verbesserung der hämodynamischen Stabilität bei chronisch systolischer Herzinsuffizienz (Angermann 2009). Das könnte also bedeuten, dass eine bestehende Adipositas bezüglich des Ausmaßes und der Progression des klinischen Bildes und der Belastbarkeit chronisch herzinsuffizienter Patienten mit eingeschränkter Pumpfunktion eine protektive Rolle spielt.

Eine Gewichtsreduktion mittels Diät und moderater Bewegung könnte die Lebensqualität und die NYHA-Klasse chronisch herzinsuffizienter Patienten einerseits optimieren, andererseits ist jedoch unklar, ob diese Maßnahmen auch das Überleben verbessern würden. Denn wie das Phänomen der "obesity paradox“ deutlich macht, ist eine moderate Adipositas bei 
chronischer Herzinsuffizienz mit einem besseren Outcome als bei Norm- oder Idealgewichtigen assoziiert (Kunju et al. 2009).

\subsubsection{Inverse Interaktionen}

Eine umgekehrte Wirkung auf die NYHA-Klasse konnte beim Vorliegen eines Hypertonus oder einer Hyperlipidämie gezeigt werden.

\section{$\underline{\text { Hypertonie }}$}

Der Hypertonus stellte in dieser Arbeit die häufigste Komorbidität chronisch herzinsuffizienter Patienten dar und erhält somit eine große Relevanz. Während eine Hypertonie bei $61,4 \%$ systolisch herzinsuffizienter Patienten vorlag, war er mit $78,4 \%$ bei herzinsuffizienten Patienten mit erhaltener Ejektionsfraktion signifikant häufiger vorzufinden. Der Hypertonus ist ein bekannter Risikofaktor für kardiovaskuläre Erkrankungen und spielt bei der Entstehung und Progression der Herzinsuffizienz eine entscheidende Rolle.

Der Einfluss einer Hypertonie bei chronischer Herzinsuffizienz wurde bisher in einigen Therapiestudien untersucht, wobei der Effekt bezüglich Mortalität, Hospitalisierung und Prognose im Mittelpunkt der Analysen stand. Außerdem wurden eher Patienten mit systolischer Herzinsuffizienz in diese Studien einbezogen. Untersuchungen zu dem Einfluss dieser Komorbidität auf die Leistungsfähigkeit und Symptomatik herzinsuffizienter Patienten sind eher rar und auch das Patientenkollektiv mit erhaltener Ejektionsfraktion wurde nur selten analysiert.

In der hier vorliegenden Studie wurde der Einfluss einer Hypertonie auf die NYHA-Klasse systolisch herzinsuffizienter Patienten im Vergleich zu herzinsuffizienten Patienten mit erhaltener Pumpfunktion, adjustiert auf Geschlecht und definierte Altersgruppen, untersucht. Bezüglich der systolischen Herzinsuffizienz kamen wir zu dem Ergebnis, dass das Vorliegen eines Hypertonus mit einer besseren NYHA-Klasse einhergeht und sich damit positiv auf die Symptomatik auswirkt. Eine Interaktion bezüglich des Geschlechts konnte nicht nachgewiesen werden. Allerdings konnte man beobachten, dass ein fortgeschrittenes Alter mit einer höheren NYHA-Klasse einhergeht.

Diese Analysen entsprechen nicht den Ergebnissen aus früheren Studien, in denen eine Therapie mit Antihypertensiva meist eine Verbesserung des klinischen Status systolisch herzinsuffizienter Patienten zur Folge hatte.

In der MERIT-HF-Studie (Hjalmarson et al. 2000) wurde der Effekt von Metoprolol versus Placebo bei systolisch herzinsuffizienten Patienten analysiert. Das Ergebnis war, dass eine Therapie mit dem Betablocker im Gegensatz zur Placebogruppe mit einer Verbesserung des 
Überlebens, einer Reduktion der Hospitalisierungsrate und mit einer Optimierung der NYHAKlasse assoziiert war (Hjalmarson et al. 2000).

Ähnlich verhielt es sich bei den Therapiestudien mit der Anwendung von ACE-Hemmern bei herzinsuffizienten Patienten mit eingeschränkter Ejektionsfraktion. Die Captopril-MultizenterStudie berichtete als erste Studie über eine Verbesserung der körperlichen Belastbarkeit und der Symptomatik, unter der Therapie mit Captopril (Captopril-Multizenter Studie 1983). Des Weiteren konnte unter $10 \mathrm{mg}$ Imidapril pro Tag ein signifikanter Anstieg der Belastbarkeitsdauer sowie der physikalischen Arbeitskapazität nachgewiesen werden (Dolezal 2006). Ebenso wurde festgestellt, dass eine Behandlung älterer Patienten mit linksventrikulärer systolischer Dysfunktion mit dem ACE-Hemmer Perindopril zu einer signifikanten Verbesserung der Distanz im 6-Minuten-Gehtest im Vergleich zur Placebogruppe führte. Aber ein Effekt auf die Lebensqualität war nicht erkennbar (Cleland et al. 2006).

In all den genannten Therapiestudien führt eine Blutdrucksenkung zur Verbesserung der Belastbarkeit chronisch systolisch herzinsuffizienter Patienten und widerspricht den Ergebnissen unserer Studie. Allerdings ist die chronisch systolische Herzinsuffizienz ein progressiver kataboler Zustand, der durch Gewichts- und Muskelreduktion, Anorexie, abnorme Glukose-, Leber- und Fettstoffwechselstörungen gekennzeichnet ist und ein ungeeignetes Zusammenspiel von Zytokinen, Neuropeptiden und Stresshormonen aufweist (Kalantar-Zadeh et al. 2007). In einem solchen Zustand wird im Rahmen des „survival paradox" bzw. der „reversen Epidemiologie“ beschrieben, dass ein arterieller Hypertonus zu einer Verbesserung der hämodynamischen Stabilität führt (Angermann 2009). Das könnte erklären, dass ein hoher Blutdruck zur Aufrechterhaltung einer gewissen Leistungsfähigkeit bei chronisch systolischer Herzinsuffizienz vonnöten ist.

Außerdem wurde in den Therapiestudien nicht genau analysiert, ob die Verbesserung der Belastbarkeit tatsächlich aus den Effekten einer Blutdrucksenkung resultiert oder ob diese Verbesserung eventuell anderen Effekten zuzuschreiben ist.

Des Weiteren haben wurde in dieser Studie die Auswirkung eines Hypertonus auf die NYHAKlasse herzinsuffizienter Patienten mit erhaltener Ejektionsfraktion, adjustiert auf Geschlecht und definierte Altersgruppen, untersucht. Ein bestehender Hypertonus hatte allerdings keinen signifikanten Einfluss auf die Symptomatik oder die NYHA-Klasse herzinsuffizienter Patienten mit erhaltener Pumpfunktion.

Dies entspricht auch den Analysen der I-Preserve-Studie (Massie et al. 2008), in der die Wirkung des AT1-Blockers Irbesartan versus Placebo auf chronisch herzinsuffiziente Patienten mit einer $E F \geq 45 \%$ untersucht wurde. Irbesartan hatte kein besseres Outcome der 
Patienten zur Folge. Allerdings ist diese Studie mit unseren Ergebnissen nur bedingt vergleichbar, da diese durch andere Endpunkte definiert wurde (Massie et al. 2008).

Im Gegensatz dazu untersuchte die PEP-CHF-Studie (Cleland et al. 2006) die Wirkung des ACE-Hemmers Perindopril im Vergleich zu einem Placebo bei chronisch herzinsuffizienten Patienten mit einer diastolischen Dysfunktion mit dem Ergebnis eines verbesserten 6Minuten-Gehtests unter Perindopril. Ebenso zeigten sie eine Optimierung der körperlichen Leistungsfähigkeit und Symptomatik sowie eine reduzierte Hospitalisierungsrate unter der Therapie mit Perindopril (Cleland et al. 2006).

In einer weiteren Studie zur Therapie mit Betablockern wurde der Einfluss von Atenolol versus Nebivolol auf Patienten mit diastolischer Herzinsuffizienz untersucht. Beide Betablocker führten zu einer Verbesserung der klinischen Symptome, was anhand der NYHA-Klasse bewertet wurde. Aber nur Nebivolol war assoziiert mit einer signifikanten Steigerung der körperlichen Leistungsfähigkeit in Form eines Anstiegs des peak $\mathrm{VO}_{2}$, der $\mathrm{VO}_{2}$ an der anaeroben Schwelle und eines Abfalls der $\mathrm{VE} / \mathrm{VCO}_{2}$. Dieser Unterschied könnte dadurch erklärt werden, dass Nebivolol infolge einer steigenden NO-Freisetzung eine Vasodilatation und Perfusion der belasteten Skelettmuskulatur und eine Optimierung der linksventrikulären diastolischen Funktion zur Folge hat (Nodari et al. 2003).

Es drängt sich also der Schluss auf, dass eine medikamentöse Drucksenkung häufig auch mit einer höheren Belastbarkeit einhergeht. Dies ist aber vielleicht anderen Effekten als dem Blutdruck-Effekt zuzuschreiben, denn offensichtlich sind in unserer Studie die Patienten mit systolischer Herzinsuffizienz besser belastbar, wenn sie einen höheren Blutdruck haben.

\section{Hyperlipidämie}

Einige Studien zeigten, dass eine Langzeittherapie mit lipidsenkenden Medikamenten die Häufigkeit einer Herzinsuffizienz bei KHK-Patienten signifikant reduzierte (Wang et al. 1999). Aber welchen Effekt eine bestehende Hyperlipidämie auf die Prognose, Mortalität und Morbidität chronisch herzinsuffizienter Patienten ausübt, ist bisher noch unbekannt.

Dies ist daher die erste Studie, die sich mit dem Einfluss einer vorhandenen Hyperlipidämie auf die Ausprägung der Symptomatik chronisch herzinsuffizienter Patienten mit erhaltener im Vergleich zu eingeschränkter Pumpfunktion beschäftigt hat. Diese Komorbidität hat eine hohe Relevanz, da sie immerhin bei 58,8\% der Patienten mit systolischer Herzinsuffizienz und bei $54 \%$ der Patienten mit Herzinsuffizienz und erhaltener Ejektionsfraktion nachzuweisen ist und damit nach der Hypertonie die zweithäufigste Komorbidität in beiden Patientengruppen darstellt.

Wir haben festgestellt, dass der Einfluss einer Hyperlipidämie bezüglich der Ejektionsfraktion variiert. Bei der systolischen Herzinsuffizienz hatte die Hyperlipidämie einen signifikant 
positiven Einfluss auf die NYHA-Klasse und bewirkte somit eine Verbesserung der Symptomatik, während eine gleichzeitig bestehende Hyperlipidämie bei der Herzinsuffizienz mit erhaltener Ejektionsfraktion eine negative Auswirkung auf die NYHA-Klasse hatte und dadurch mit einer schwereren Herzinsuffizienzsymptomatik einherging. Bezüglich des Geschlechts bestand allerdings kein signifikanter Zusammenhang.

Die Daten über die Auswirkungen bei der HFREF könnte man mit den Ergebnissen des „obesity paradox"-Phänomens erklären, das ein besseres Outcome unter den übergewichtigen herzinsuffizienten Patienten im Vergleich zu Normgewichtigen beschreibt. Denn Lipidstoffwechselstörungen sind häufig mit anderen Erkrankungen wie der Adipositas assoziiert (Herold 2008). Und unsere Ergebnisse zur HFPEF entsprechen in etwa unseren Resultaten zur Adipositas, die einen signifikant negativen Effekt auf die NYHA-Klasse der HFPEF hatte. Es stellt sich also die Frage, ob eine lipidsenkende Therapie oder diätische Maßnahmen einen positiven Effekt auf die Symptomatik herzinsuffizienter Patienten hat, oder ob systolisch herzinsuffiziente Patienten grundsätzlich eine andere Therapie bezüglich der Hyperlipidämie erhalten sollten als herzinsuffiziente Patienten mit erhaltener Ejektionsfraktion.

\subsubsection{Der Stellenwert der Komorbiditäten bezüglich der Symptomatik chronisch herzinsuffizienter Patienten im Vergleich zu Parametern der ventrikulären systolischen und diastolischen Funktion}

Die Hauptsymptome der chronischen Herzinsuffizienz, ob mit erhaltener oder eingeschränkter Ejektionsfraktion, sind Dyspnoe, Müdigkeit und Einschränkungen der körperlichen Leistungsfähigkeit. Bei der Entstehung dieser Symptome spielen vor allem diastolische Parameter (LVD(ED)) und der erhöhte linksventrikuläre Füllungsdruck, der auch bei der systolischen Herzinsuffizienz bekannt ist, eine große Rolle. Allerdings ist der Einfluss anderer pathophysiologischer Parameter auf die Belastbarkeit teilweise noch größer. So bewirken eine Reduktion der kardialen Auswurfleistung und eine pulmonale Hypoperfusion ebenfalls Belastungseinschränkungen und Dyspnoe. Neben der Senkung der kardialen Auswurfleistung erfolgen durch die Aktivierung des Renin-Angiotensin-Systems und den steigenden Sympathikotonus eine periphere Vasokonstriktion und damit zusätzlich eine Umverteilung des Blutflusses zu Herz und Gehirn (Stassijns et al. 1996). Die reduzierte Durchblutung vor allem der Skelettmuskulatur hat typische Veränderungen zur Folge: Es kommt zu einer Reduktion und Atrophie der Muskelmasse und zu pathologischen Veränderungen von Struktur, Stoffwechsel und Funktion des Muskels, die eine zunehmende Belastungsintoleranz bewirken (Volterrani et al. 1994). 
Auch proinflammatorische Zytokine stören den Metabolismus der Skelettmuskulatur, indem sie eine Atrophie induzieren. Außerdem spielen sie eine zentrale Rolle in der Entstehung der kardialen Kachexie (Itoh et al. 2005). Die respiratorischen Muskeln sind ebenfalls betroffen: Die Muskelarbeit steigt, denn die Muskelfunktion wird infolge kontraktiler Eigenschaften, reduzierter Durchblutung und histologischen sowie histochemischen Veränderungen stark eingeschränkt (Stassijns et al. 1996). Und auch die Compliance der Lungen nimmt in Folge einer zunehmenden Versteifung durch Kongestion und resultierender Ödeme ab und begünstigt ebenfalls die Entstehung von Dyspnoe und Einschränkungen der körperlichen Leistungsfähigkeit (Wilson und Mancini 1993).

Allerdings findet man bei chronisch herzinsuffizienten Patienten neben der Herzinsuffizienz selbst noch einige andere Erkrankungen. Die Bedeutung solcher Komorbiditäten bezüglich des Einflusses auf die Ausprägung der Herzinsuffizienzsymptomatik wurde in dieser Studie untersucht. Wir konnten zeigen, dass die Komorbiditäten einen großen Stellenwert in der Klinik der Herzinsuffizienz einnehmen und sogar einen signifikanten Einfluss ausüben.

Da Komorbiditäten selten solitär auftreten, wurde der Einfluss der Komorbiditäten auch in Anwesenheit anderer Begleiterkrankungen überprüft. Das Ausmaß des Einflusses der Komorbiditäten wurde mit dem Ausmaß des Einflusses von linksventrikulärer Funktion (LVEF) und dem linksventrikulären enddiastolischen Durchmesser (LVD(ED)) verglichen. Bei der HFREF hatten dabei Alter, weibliches Geschlecht, Diabetes mellitus, renale Dysfunktion, Hyperurikämie, Anämie, COPD und ein LVEF-Abfall einen signifikant negativen Einfluss auf die NYHA-Klasse, wobei die Abhängigkeit der NYHA-Klasse von der LVEF etwa dem Einfluss der renalen Dysfunktion oder der Hyperurikämie entsprach. Bei der HFPEF gingen nur das Alter, weibliches Geschlecht, Anämie, COPD, KHK und eine Abnahme des LVD(ED) mit einer schlechteren NYHA-Klasse einher.

Die multivariaten Analysen konnten also den hohen Stellenwert der Komorbiditäten bezüglich des klinischen Status und der Ausprägung der Herzinsuffizienzsymptomatik bestätigen. Außerdem konnte gezeigt werden, dass der Einfluss der Komorbiditäten bezüglich der NYHA-Klasse größer ist als der Effekt durch die Veränderungen relevanter linksventrikulärer Parameter wie dem LVD(ED) und der LVEF.

Hieraus wird deutlich, dass die Diagnose von Komorbiditäten eine große und vielleicht unterschätzte Bedeutung für die Belastbarkeit herzinsuffizienter Patienten hat. Denn ein Progress einer Herzinsuffizienz und die Ausprägung der Symptomatik könnten verbessert werden, wenn neben der Behandlung der Herzinsuffizienz auch die verschiedenen Komorbiditäten als therapeutisches Ziel streng verfolgt würden. Besonders die Gewichtung der Komorbiditäten in der multivariaten Analyse gegenüber den pathophysiologischen Parametern spricht für einen therapeutischen Ansatz, der die Behandlung von 
Diskussion

Komorbiditäten fokussiert, um die Symptome und Belastbarkeit zu verbessern. Für eine Verifikation dieser Vermutung sind allerdings weitere Studien nötig. 


\section{Zusammenfassung}

Die Therapie der Herzinsuffizienz mit erhaltener Ejektionsfraktion (HFPEF) ist im Vergleich zur standardisierten und evidenzbasierten Therapie der Herzinsuffizienz mit reduzierter EF (HFREF) bisher nur symptomatisch und an Grundkrankheiten orientiert. Der Einfluss verschiedener Komorbiditäten ist für die HFPEF wenig untersucht. Deshalb wurde in der vorliegenden Arbeit untersucht, inwiefern ausgewählte Komorbiditäten die Symptomatik und den klinischen Schweregrad der HFPEF und HFREF unterschiedlich beeinflussen.

Aus elf Teilprojekten des KNHI konnten 4079 herzinsuffiziente Patienten, aufgeteilt in HFREF ( $E F<50 \%, n=2785)$ und HFPEF $(E F \geq 50 \%, n=1294)$, in die Analyse einbezogen werden (mittleres Alter 67 Jahre, 66\% männlich). Patienten mit HFPEF waren im Vergleich zu Patienten mit HFREF durch ein höheres Alter, überwiegend weibliches Geschlecht, höheren Blutdruck, niedrigere Herzfrequenz in Ruhe und eine seltenere Einnahme kardiovaskulärer Medikamente gekennzeichnet. Insgesamt waren die NYHA-Klassen II und III im Gesamtkollektiv am häufigsten vertreten. Die Symptomlast entsprechend der Framinghamkriterien war in beiden Gruppen ähnlich, jedoch zeigten die Patienten mit eingeschränkter EF höhere NYHA-Klassen.

Ausgewählte Komorbiditäten wurden ermittelt und deren Einfluss auf die Ausprägung der Herzinsuffizienzsymptomatik anhand der NYHA-Klasse überprüft. Jede der geprüften Komorbiditäten war im Mittel bei 37\% (15-61\%) der Patienten mit HFREF und bei 33\% der Patienten mit HFPEF (13-78\%) nachweisbar. Davon fanden sich Hypertonie und Adipositas signifikant häufiger bei Patienten mit HFPEF, während Diabetes mellitus, Hyperurikämie, renale Dysfunktion, Anämie, COPD, KHK und Hyperlipidämie in der Gruppe der HFREF signifikant häufiger auftraten.

Einen vergleichbar negativen Einfluss auf die NYHA-Klasse bei HFPEF und HFREF zeigten Diabetes mellitus, Hyperurikämie, renale Dysfunktion und Anämie: Unabhängig von der EF bestand eine signifikante negative Assoziation zwischen den genannten Komorbiditäten und der NYHA-Klasse.

Eine vorhandene COPD hatte unterschiedliche Effekte auf die NYHA-Klasse in Abhängigkeit vom Geschlecht: Die COPD verschlechterte die NYHA-Klasse stärker bei Männern, jedoch ohne Interaktion mit der LVEF.

Unterschiedliche Interaktionen fanden sich bezüglich KHK, Adipositas und Hypertonie. KHK und Adipositas waren nur in der Gruppe der HFPEF mit einer Verschlechterung der NYHAKlasse assoziiert, nicht jedoch in der Gruppe der HFREF. Ein bestehender oder bekannter Hypertonus beeinflusste die NYHA-Klasse bei Patienten mit HFREF positiv, zeigte jedoch keinen signifikanten Einfluss auf die Symptomatik der Patienten mit erhaltener Pumpfunktion. 
Eine vorhandene Hyperlipidämie beeinflusste die NYHA-Klasse bei HFREF positiv, während diese Komorbidität bei HFPEF mit einer höheren NYHA-Klasse assoziiert war.

In der multivariaten Analyse wurde deutlich, dass bei der HFREF alle Komorbiditäten, die einzeln einen negativen Effekt auf die Belastbarkeit ausüben, diesen auch bei gleichzeitig vorkommenden Erkrankungen beibehalten. Das Ausmaß des negativen Effektes der einzelnen, im multivariaten Modell geprüften Komorbiditäten war mit dem Ausmaß einer weiteren Reduktion der LVEF um 10\% vergleichbar. Bei Patienten mit HFPEF bestand im multivariaten Modell nur für Alter, weibliches Geschlecht, Anämie, COPD, KHK und Adipositas ein signifikant negativer Effekt auf die NYHA-Klasse. Dieser war jedoch deutlich größer als der Effekt durch eine Verminderung des enddiastolischen linksventrikulären Durchmessers (LVD(ED)).

Zusammenfassend zeigten sich bei Patienten mit HFREF und HFPEF neben demographischen Unterschieden auch Unterschiede bezüglich der klinischen Charakteristika sowie der Verteilung von Komorbiditäten. Die Patienten mit HFPEF waren insgesamt weniger symptomatisch. Verglichen mit HFREF wirkten sich einzelne Komorbiditäten bei HFPEF unterschiedlich stark auf die Schwere der Symptomatik aus. Vor allem das Ausmaß des negativen Effektes von diesen Komorbiditäten ist, verglichen mit einem pathophysiologisch wichtigen Leitparameter, bei Patienten mit HFPEF größer als bei HFREF. Dies deutet möglicherweise auf einen größeren relativen Einfluss bestimmter Komorbiditäten auf die Ausprägung der Symptomatik bei Patienten mit HFPEF hin und sollte daher bei der Evaluation und Behandlung dieser Patienten berücksichtigt werden. 


\section{Anhang}

\subsection{Abkürzungsverzeichnis}

\begin{tabular}{|c|c|}
\hline ACE & Angiotensin-converting-Enzym \\
\hline ANP & atriales natriuretisches Peptid \\
\hline AT1 & Angiotensin-II-Rezeptor Typ 1 \\
\hline AV & atrioventrikulär \\
\hline BMI & body mass index \\
\hline BNP & brain natriuretic peptide \\
\hline $\mathrm{CHF}$ & congestive heart failure \\
\hline COPD & chronisch-obstruktive Lungenerkrankungen \\
\hline CRP & C-reaktives Protein \\
\hline$d$ & Tag \\
\hline $\mathrm{dl}$ & Deziliter \\
\hline EF & Ejektionsfraktion \\
\hline EKG & Elektrokardiogramm \\
\hline EPO & Erythropoetin \\
\hline FEV1 & forcierte Einsekundenkapazität \\
\hline g & Gramm \\
\hline GFR & glomeruläre Filtrationsrate \\
\hline $\mathrm{HbA}_{1 \mathrm{c}}$ & Glykohämoglobin \\
\hline $\mathrm{HF}$ & Herzinsuffizienz \\
\hline HFNEF & Herzinsuffizienz mit normaler Ejektionsfraktion \\
\hline HFPEF & Herzinsuffizienz mit erhaltener Ejektionsfraktion \\
\hline HFREF & Herzinsuffizienz mit reduzierter Ejektionsfraktion \\
\hline HIV & humanes Immundefizienzvirus \\
\hline $\mathrm{HKU}$ & Herzkatheteruntersuchung \\
\hline HMG-CoA & 3-Hydroxy-3-Methylglutaryl-Coenzym-A \\
\hline i.v. & intravenös \\
\hline IVSED & enddiastolischer Durchmesser des intervenrikulären Septums \\
\hline $\mathrm{kg}$ & Kilogramm \\
\hline KHK & koronare Herzkrankheit \\
\hline $\mathrm{KI}$ & Konfidenzintervall \\
\hline $\mathrm{KNHI}$ & Kompetenznetz Herzinsuffizienz \\
\hline I & Liter \\
\hline LAES & linksatriale endsystolische Größe \\
\hline
\end{tabular}


LV

LVD(ED)

LVDES

LVEDP

LVEDV

LVEF

LVSD

m

$\mathrm{m}^{2}$

$\mathrm{mg}$

$\min$

$\mathrm{ml}$

$\mathrm{mm}$

$\mathrm{mmHg}$

n

nmol

NT-proBNP

NYHA

OR

pAVK

peak $\mathrm{VO}_{2}$

PLUM

PWED

p-Wert

RAAS

RV

SD

VE

$\mathrm{VE} / \mathrm{VCO}_{2}$

$\mathrm{VO}_{2}$

$\mathrm{VO}_{2 \max }$

w linksventrikulär, linker Ventrikel

linksventrikulärer enddiastolischer Durchmesser

linksventrikulärer endsystolischer Durchmesser

linksventrikulärer enddiastolischer Druck

linksventrikuläres enddiastolisches Volumen

linksventrikuläre Ejektionsfraktion

linksventrikuläre systolische Dysfunktion

männlich

Quadratmeter

Milligramm

Minute

Milliliter

Millimeter

Millimeter Quecksilbersäule

Häufigkeit

Nanomol

$\mathrm{N}$-terminales Fragment der Vorform des brain natriuretic peptide

New York Heart Association

Odds Ratio

periphere arterielle Verschlusskrankheit

Sauerstoffaufnahme bei maximaler Belastung

Polytomous Universal Model

enddiastolische Hinterwanddicke

Signifikanzwert

Renin-Angiotensin-Aldosteron-System

rechter Ventrikel

Standardabweichung

Atemminutenvolumen

Atemäquivalent für $\mathrm{CO}_{2}$

Sauerstoffaufnahme

maximale Sauerstoffaufnahme

weiblich 


\section{2 Übersicht über die teilhabenden Teilprojekte des KNHI (erstellt mit Hilfe von Dr. Christiane Prettin, ZKS Leipzig - KKS)}

\section{TP5: Gen-Studie}

\begin{tabular}{|c|c|}
\hline Titel der Studie: & $\begin{array}{l}\text { Teilprojekt } 5 \text { : Ätiologie der Herzinsuffizienz Im Kompetenznetz } \\
\text { Herzinsuffizienz }\end{array}$ \\
\hline Kurzbezeichnung der Studie: & Genstudie \\
\hline Gegenstand der Studie & $\begin{array}{l}\text { Die dilatative (DCM) und hypertrophe }(\mathrm{HCM}) \text { Kardiomyopathien sind } \\
\text { in der Regel primär vom Myokard ausgehende Erkrankungen und } \\
\text { können familiär gehäuft auftreten ( } \mathrm{DCM}<\mathrm{HCM}) \text {. Obwohl für } \mathrm{DCM} \text { und } \\
\text { mehr noch für HCM zahlreiche genetische Befunde bekannt sind, ist } \\
\text { die relative Häufigkeit von Krankheitsgenen und die Genotyp- } \\
\text { Phänotypkorrelation nur an kleinen Patientengruppen untersucht. }\end{array}$ \\
\hline Primäres Ziel der Studie: & $\begin{array}{l}\text { Primäres Ziel des Teilprojekts ist es die Häufigkeit von } \\
\text { Krankheitsgenen bzw. Mutationen in den Krankheitsgenen in zwei } \\
\text { großen Patientenkollektiven (HCM-Kollektiv und DCM-Kollektiv) zu } \\
\text { ermitteln. }\end{array}$ \\
\hline Sekundäre Ziele der Studie: & $\begin{array}{l}\text { Sekundäres Ziel der Studie ist es, die klinischen Daten mit den } \\
\text { genetischen Befunden zusammenzuführen und damit erstmals mit } \\
\text { Hilfe großer Fallzahlen valide Daten zu Genotyp-Phänotyp-Relationen } \\
\text { insbesondere im Hinblick auf eine geschlechtsspezifische Penetranz } \\
\text { der Erkrankung herzustellen. }\end{array}$ \\
\hline Studiendesign: & $\begin{array}{l}\text { Es handelt sich um eine offene, multizentrische, vergleichende } \\
\text { Registerstudie. }\end{array}$ \\
\hline Studienpopulation: & $\begin{array}{l}\text { Einschlusskriterien DCM (Mestroni et al. 1999b) } \\
\text { Patienten mit eingeschränkter systolischer Pumpfunktion } \\
\text { Echo: } \\
\text { EF < } 45 \% \text { und/oder FS < } 25 \% \\
\text { LVEDD > } 117 \% \text { (Korrigiert nach Alter und Körperoberfläche) } \\
\qquad \text { Henry Gleichung: [ } 45,3 \times \text { BSA } 1 / 3-0,03(\text { age) - 7,2] } \\
\text { Ausschlusskriterien DCM } \\
\text { - langjährige arterielle Hypertonie mit Veränderungen des Augen- } \\
\quad \text { hintergrundes } \\
\text { - RR > 160/100 mm Hg (bei mehreren Messungen) } \\
\text { - KHK (Lumeneinengung > } 50 \% \text { eines Gefäßes } \\
\text { - C2-Abusus (w > 40g/Tag - m > } 80 \mathrm{~g} / T \text { Tag mehr als } 5 \text { Jahre } \\
\text { - anhaltende schnelle supraventrikuläreArrhythmien } \\
\text { - bekannte Systemerkrankung } \\
\text { - Perikarderkrankung } \\
\text { - angeborene Herzerkrankung } \\
\text { - Cor pulmonale } \\
\text { Einschlusskriterien HCM (McKenna et al. 1997) } \\
\text { Patienten mit echokardiographischer Hypertrophie des linken oder } \\
\text { beider Ventrikel. }\end{array}$ \\
\hline
\end{tabular}




\begin{tabular}{|c|c|}
\hline & $\begin{array}{l}\text { Echo: } \\
\text { PWED (posteriore Wand des linken Ventrikels, endiastolisch) } \geq 13 \\
\text { mm } \\
\text { IVSED (interventrikuläres Septum, endiastolisch ) } \geq 15 \mathrm{~mm} \\
\text { Ausschlusskriterien HCM } \\
\text { - langjährige arterielle Hypertonie mit Veränderungen des Augen- } \\
\text { hintergrundes } \\
\text { - RR > 160/100 mm Hg (bei mehreren Messungen) } \\
\text { - Valvuläre Aortenstenose } \\
\text { - bekannte Systemerkrankung }\end{array}$ \\
\hline Patientenzahl: & $\begin{array}{l}\text { In die Studie sollen } 600 \text { Patienten mit hypertrophischer } \\
\text { Kardiomyopathie (HCM Kollektive) und } 900 \text { Patienten mit dilatativer } \\
\text { Kardiomyophatie (DCM Kollektiv) eingeschlossen werden. }\end{array}$ \\
\hline Therapie: & - entfällt- \\
\hline Primärer Endpunkt: & $\begin{array}{l}\text { Prävalenz der bekannten Krankheitsgene bei Patienten mit DCM und } \\
\text { HCM. }\end{array}$ \\
\hline Sekundäre Endpunkte: & $\begin{array}{l}\text { Klinische Phänomene werden speziellen Mutationen in } \\
\text { Krankheitsgenen zugeordnet. }\end{array}$ \\
\hline Biometrie: & $\begin{array}{l}\text { Bei der Auswertung bzgl. des primären Endpunktes finden Methoden } \\
\text { der explorativen Datenanalyse Anwendung. Die berechneten relativen } \\
\text { Häufigkeiten werden mit dem dazugehörenden } 95 \% \text { - } \\
\text { Konfidenzintervall }(\mathrm{Cl}) \text { angegeben. Gegebenenfalls erfolgen diese } \\
\text { Angaben stratifiziert nach Basismerkmalen, sofern sich dort } \\
\text { Abhängigkeiten zeigen.Für die Auswertung des sekundären } \\
\text { Endpunktes finden Zusammenhangsanalysen, wie Korrelation und } \\
\text { Odds-Ratio mit } 95 \%-\mathrm{Cl} \text {, Anwendung. }\end{array}$ \\
\hline Zeitplan: & $\begin{array}{l}\text { Patientenbezogen: je eine Untersuchung und Blutabnahme im } \\
\text { Rahmen der Routineblutabnahmen } \\
\text { kein follow up } \\
\text { Studienbezogen: } \\
\text { Beginn: } 8 / 2004 \text {; Rekrutierungsende: } 6 / 2006\end{array}$ \\
\hline
\end{tabular}




\section{TP6: Handheld-BNP}

\begin{tabular}{|c|c|}
\hline Titel der Studie: & $\begin{array}{l}\text { Objektivierung der kardiovaskulären Dysfunktion im ambulanten und } \\
\text { hausärztlichen Bereich mittels handgehaltener Echokardiographie und } \\
\text { dem BNP-Schnelltest. Multicenter-Studie des Teilprojekts } 6 \text { im } \\
\text { Kompetenznetz Herzinsuffizienz }\end{array}$ \\
\hline Kurzbezeichnung: & Handheld-BNP-Studie \\
\hline Indikation: & Herzinsuffizienzdiagnostik \\
\hline Einschlusskriterien & $\begin{array}{l}\text { - Verdacht auf das Vorliegen einer Herzinsuffizienz ausschließlich } \\
\text { aufgrund anamnestischer Angaben des Patienten/der Patientin } \\
\text { (es dürfen noch keine objektivierenden Untersuchungen } \\
\text { durchgeführt worden sein!) } \\
\text { - Vorliegen einer schriftlichen Einverständniserklärung des } \\
\text { Patienten/der Patientin zur Studienteilnahme nach erfolgter } \\
\text { schriftlicher und mündlicher Aufklärung durch den Hausarzt } \\
\text { - Alter } \geq 18 \text { Jahre }\end{array}$ \\
\hline Ausschlusskriterien & 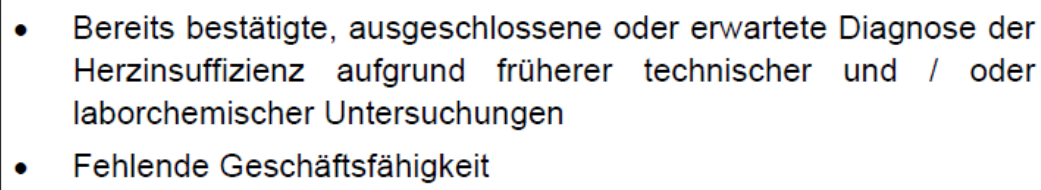 \\
\hline Primäres Ziel der Studie: & $\begin{array}{l}\text { Untersuchung, ob Primärdiagnostik der Herzinsuffizienz mit Hilfe von } \\
\text { BNP-Schnelltests und/oder handgehaltener Echokardiographie } \\
\text { verbessert werden kann }\end{array}$ \\
\hline Sekundäre Ziele der Studie: & $\begin{array}{l}\text { Separate Analyse: Eignung der handgehaltenen Echokardiographie } \\
\text { sowie des BNP-Schnelltests in der Primärdiagnostik der } \\
\text { Herzinsuffizienz durch den Hausarzt; } \\
\text { Konkordanz des BNP-Schnelltests mit Goldstandard; } \\
\text { Schätzung des Trainingsaufwands für eine korrekte Diagnose-stellung } \\
\text { mit beiden Methoden }\end{array}$ \\
\hline Studiendesign: & Vierarmig, kontrolliert, cluster-randomisiert, offen, multizentrisch \\
\hline Studienpopulation: & $\begin{array}{l}\text { Patienten mit Verdacht auf Herzinsuffizienz, die beim Hausarzt } \\
\text { vorstellig werden }\end{array}$ \\
\hline Fallzahl: & 960 Patienten / 48 Hausärzte \\
\hline Diagnose-Modalitäten: & $\begin{array}{l}\text { 1. Klinik, 2. Klinik+Triage }{ }^{\circledR} \text { BNP-Test, 3. Klinik+Handheld-Echo, } 4 . \\
\text { Klinik+Triage }{ }^{\circledR} \text { BNP-Test+Handheld-Echo }\end{array}$ \\
\hline Primärer Endpunkt: & Konkordanz/Diskordanz der Diagnosen von Hausarzt und Kardiologe \\
\hline Sekundäre Endpunkte: & $\begin{array}{l}\text { BNP aus Schnelltest und Referenzstandard; } \\
\text { Trainingszeit der Hausärzte in der Trainingsstudie }\end{array}$ \\
\hline Biometrie: & $\begin{array}{l}\text { Primäre Fragestellung: t-Test für einen Scorewert (per Hausarzt), der } \\
\text { die Überlegenheit der Ausstattung mit Zusatzdiagnostik misst; } \\
\text { Sekundär: Schätzer für einzelne Sensitivitäten, Spezifitäten, PPV, } \\
\text { NPV und deren Differenzen zwischen den Diagnostik-Modalitäten; } \\
\text { Bland-Altman-Analyse für BNP-Bestimmungen; } \\
\text { Schätzung von Verteilungsparametern der Trainingszeit }\end{array}$ \\
\hline
\end{tabular}


Anhang

Zeitplan:

Patientenbezogen: Nach Erstvorstellung beim Hausarzt erfolgt innerhalb von 2 Wochen die Nachuntersuchung durch den Kardiologen.

Studienbezogen: Beginn 11/2003, Rekrutierungsdauer 20 Monate, voraussichtlicher Abschluß 12/2005. 


\section{TP7: Diast-CHF}

\begin{tabular}{|c|c|}
\hline Titel der Studie: & $\begin{array}{l}\text { PRÄVALENZ UND VERLAUF DER DIASTOLISCHEN DYSFUNKTION } \\
\text { UND DER DIASTOLISCHEN HERZINSUFFIZIENZ }\end{array}$ \\
\hline Kurzbezeichnung der Studie: & Diast-CHF \\
\hline Indikation: & $\begin{array}{l}\text { Patienten mit Risikofaktoren für die Entstehung einer diastolischen } \\
\text { Dysfunktion (Gruppe A) bzw. Patienten mit Herzinsuffizienz- } \\
\text { symptomatik (Gruppe B) }\end{array}$ \\
\hline Einschlusskriterien & $\begin{array}{l}\text { - arterielle Hypertonie } \\
\text { - Diabetes mellitus } \\
\text { - Schlafapnoesyndrom } \\
\text { - Zeichen der Arteriosklerose (definiert als symptomatische pAVK, } \\
\text { angiographisch nachgewiesene KHK, Carotisstenose, Z. n. } \\
\text { Apoplex oder Z. n. Myokardinfarkt) } \\
\text { - dokumentierte ärztlich gestellte Diagnose Herzinsuffizienz } \\
\text { - Alter zwischen } 50 \text { und } 85 \text { Jahren } \\
\text { - schriftlich Ihr Einverständnis erklärt haben }\end{array}$ \\
\hline Ausschlusskriterien & $\begin{array}{l}\text { - } \text { mangelnde Kooperationsbereitschaft (Compliance) } \\
\text { - Unzureichende Kommunikationsfähigkeit (schlechte Deutsch- } \\
\text { - } \quad \text { Enntnisse, Aphasien) } \\
\text { - geographische Gründe, die eine Studienteilnahme unmöglich } \\
\text { machen }\end{array}$ \\
\hline Primäres Ziel der Studie: & $\begin{array}{l}\text { Prävalenz und Verlauf von Patienten mit diastolischer Dysfunktion bzw. } \\
\text { diastolischer Herzinsuffizienz in einem Risikokollektiv }\end{array}$ \\
\hline Sekundäre Ziele der Studie: & $\begin{array}{l}\text { Evaluation neuroendokriner Marker in Diagnostik und Verlauf der } \\
\text { diastolischen Dysfunktion/Herzinsuffizienz } \\
\text { Einfluss einer prädiabetischen Stoffwechsellage (IGT/IFG) auf den } \\
\text { Verlauf der diastolischen Dysfunktion } \\
\text { Evaluation psychosozialer Parameter in Diagnostik und Verlauf } \\
\text { Identifikation potentieller modifier SNPs mit Bedeutung für die } \\
\text { Entwicklung einer diastolischen Dysfunktion } \\
\text { Einfluss von Apnoephasen auf Ausprägung und Verlauf einer } \\
\text { diastolischen Funktionsstörung }\end{array}$ \\
\hline Studiendesign: & prospektive, offene Multicenterstudie \\
\hline Studienpopulation: & $\begin{array}{l}\text { Gruppe A: Patienten zwischen } 50 \text { und } 85 \text { Jahren mit Risikofaktoren für } \\
\text { eine diastolische Funktionsstörung (art. Hypertonie, Diab. mellitus, } \\
\text { Schlafapnoesyndrom), aber ohne klinische Zeichen der } \\
\text { Herzinsuffizienz } \\
\text { Gruppe B: Patienten mit Herzinsuffizienz (entweder bereits } \\
\text { diagnostiziert oder mit klinischen Zeichen der Herzinsuffizienz) }\end{array}$ \\
\hline Patientenzahl: & Gruppe A: 1000 Patienten, Gruppe B: 660 Patienten \\
\hline Therapie: & Keine spezifische therapeutische Intervention vorgesehen. \\
\hline Primärer Endpunkt: & $\begin{array}{l}\text { 1. Kombinierter Endpunkt aus Manifestation (Gruppe A) bzw. } \\
\text { Verschlechterung (Gruppe B) einer Herzinsuffizienz-Symptomatik, } \\
\text { Auftreten eines kardiovaskulären Ereignisses oder } \\
\text { kardiovaskulärem Tod }\end{array}$ \\
\hline
\end{tabular}




\begin{tabular}{|c|c|}
\hline & $\begin{array}{l}\text { 2. Abnahme der Belastbarkeit im 6-Minuten-Gehtest um mindestens } 15 \\
\%\end{array}$ \\
\hline Sekundäre Endpunkte: & $\begin{array}{l}\text { - Jeder einzelne Endpunkt der primären Endpunkte } \\
\text { - Verschlechterung der NYHA-Klasse um mindestens einen Punkt } \\
\text { (Studie B) } \\
\text { - Verschlechterung der diastolischen Funktionsparameter um } \\
\text { mindestens eine ASE-Klasse } \\
\text { - Veränderung von psychosozialem Befinden und Lebensqualität } \\
\text { - Gesamtmortalität }\end{array}$ \\
\hline Biometrie: & Prävalenz- und Inzidenzschätzer mit 95 \% Konfidenzintervall \\
\hline Zeitplan: & $\begin{array}{l}\text { Rekrutierung vom } 01.07 .2004 \text { bis } 30.06 .2005 \text { vorgesehen, bei nicht } \\
\text { ausreichendem Rekrutierungserfolg Verlängerung bis maximal } \\
30.09 .2005 \text { möglich, follow-up nach } 12 \text { und } 24 \text { Monaten. }\end{array}$ \\
\hline
\end{tabular}




\section{TP7: Ex-DHF-P}

\begin{tabular}{|c|c|}
\hline Titel der Studie: & $\begin{array}{l}\text { EXERCISE TRAINING IN DIASTOLIC HEART FAILURE- PILOTSTUDY } \\
\text { "A prospective, randomised, controlled study to determine the effects of } \\
\text { physical training on exercise capacity and quality of life" }\end{array}$ \\
\hline Kurzbezeichnung der Studie: & Ex-DHF-P \\
\hline Indikation: & diastolische Herzinsuffizienz \\
\hline Primäres Ziel der Studie: & $\begin{array}{l}\text { Verbesserung der körperlichen Belastbarkeit durch kontrolliertes } \\
\text { körperliches Training bei Patienten mit diastolischer Herzinsuffizienz. }\end{array}$ \\
\hline Sekundäre Ziele der Studie: & $\begin{array}{l}\text { Verbesserung verschiedener Parameter der Lebensqualität, der } \\
\text { körperlichen Belastbarkeit, der diastolischen Funktion sowie weiterer } \\
\text { krankheitsrelevanter Größen durch kontrolliertes körperliches Training bei } \\
\text { Patienten mit diastolischer Herzinsuffizienz. Erfassung von Faktoren zur } \\
\text { Trainingsadhärenz. }\end{array}$ \\
\hline Studiendesign: & prospektive, randomisierte, kontrollierte, multizentrische Studie \\
\hline Studienpopulation: & 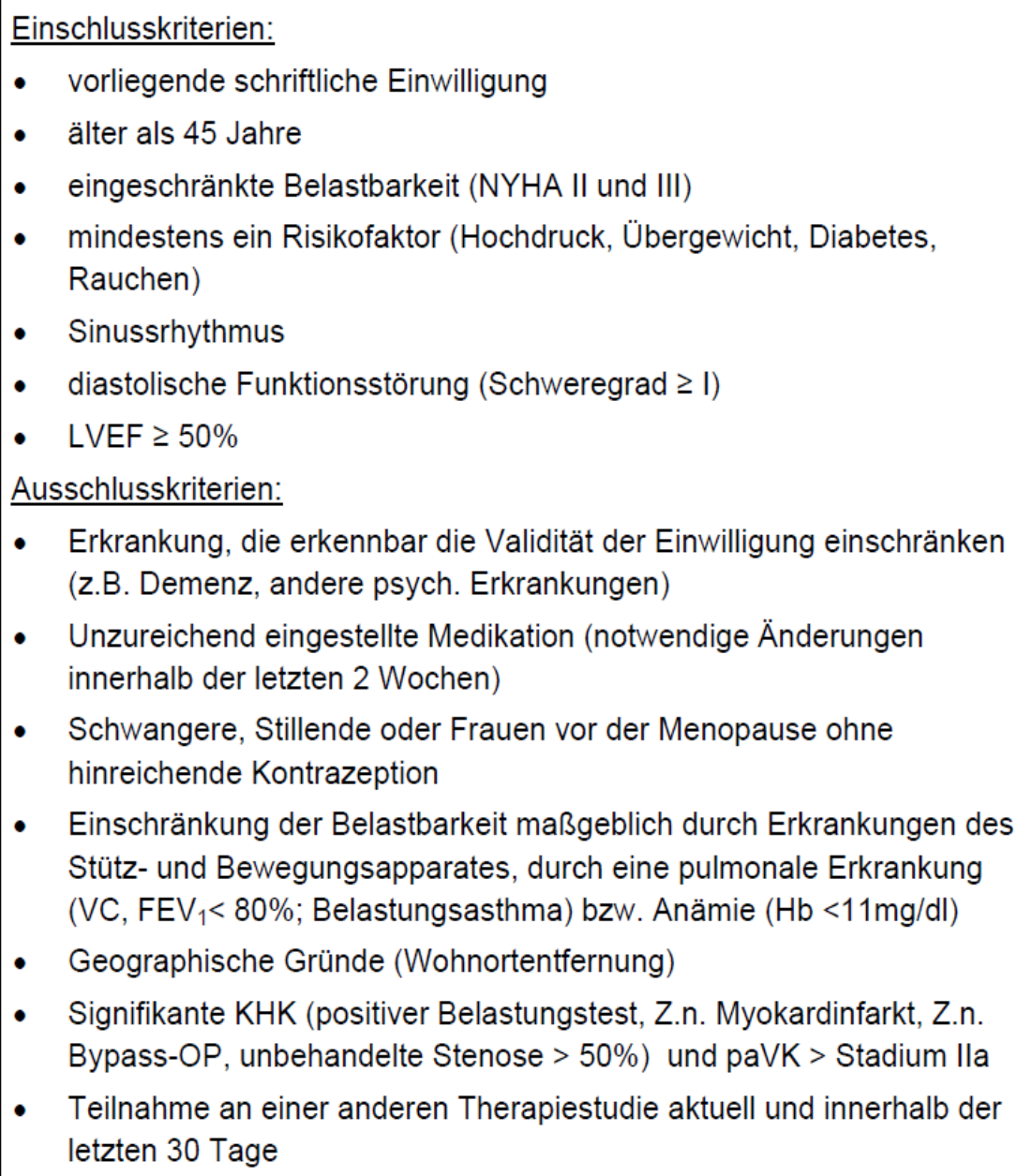 \\
\hline Patientenzahl: & 60 \\
\hline Therapie: & $\begin{array}{l}\text { Kontrollierte Trainingstherapie }(n=40) \text { versus Kontrollgruppe ohne Training } \\
(n=20) \text { für } 3 \text { Monate, Trainingsmöglichkeit für weitere } 3 \text { Monate für alle } \\
\text { Patienten. }\end{array}$ \\
\hline
\end{tabular}




\begin{tabular}{|c|c|}
\hline Primärer Endpunkt: & $\begin{array}{l}\text { Änderung der maximalen Belastbarkeit (peak } \mathrm{VO}_{2} \text {, } \\
\text { Spiroergometrie) nach } 3 \text { Monaten im Vergleich zur Baseline }\end{array}$ \\
\hline Sekundäre Endpunkte: & $\begin{array}{l}\text { 1. Änderung der peak } \mathrm{VO}_{2} \text { nach } 6 \text { Monaten } \\
\text { 2. Änderung der Lebensqualität nach } 3 \text { und } 6 \text { Monaten im } \\
\text { Vergleich zur Baseline (Minnesota, SF-36) } \\
\text { 3. Änderung von } \mathrm{E} / \mathrm{e} \text { (als Indikator des LVEDP) nach } 3 \text { und } 6 \\
\text { Monaten } \\
\text { 4. Änderung der anaeroben Schwelle (Spiroergometrie), } \\
\text { Änderung der } \mathrm{V}_{\mathrm{E}} / \mathrm{VCO}_{2} \text { slope nach } 3 \text { und } 6 \text { Monaten } \\
\text { 5. Änderung der neuroendokrinen Aktivierung (natriuretische } \\
\text { Peptide) und Änderung der Marker des myokardialen Kollagen } \\
\text { Umsatzes (PIIINP; MMPs, TIMPs) nach } 3 \text { und } 6 \text { Monaten } \\
\text { 6. Änderung der Parameter der gestörten Insulinsensitivität und } \\
\text { Inflammation (Nüchtern-Glukose, Proinsulin, Glukagon, Insulin, } \\
\text { Adiponektin, Leptin, Resistin, TNFa, IL-6, IL-1) nach } 3 \text { und } 6 \\
\text { Monaten } \\
\text { 7. Änderung der Endothelfunktion (flußvermittelte Dilatation, NO- } \\
\text { abhängig und NO-unabhängig) nach } 3 \text { und } 6 \text { Monaten }\end{array}$ \\
\hline $\begin{array}{l}\text { Begleitende sekundäre } \\
\text { Endpunkte: }\end{array}$ & $\begin{array}{l}\text { 1. Änderung einzelner echocardiographischer Parameter der } \\
\text { diastolischen Funktion (E/A, EDT, IVRT, S/D, AR, V } V_{p} \text {, TEI- } \\
\text { Index, LV mass; LA area) und des echokardiographischen } \\
\text { Grades der diastolischen Dysfunktion nach } 3 \text { und } 6 \text { Monaten } \\
\text { 2. Änderung verschiedener Parameter der Lebensqualität nach } 3 \\
\text { und } 6 \text { Monaten } \\
\text { 3. Änderung des Borg score nach } 3 \text { und } 6 \text { Monaten } \\
\text { 4. Änderung der Parameter der sympatho-vagalen Dysbalance } \\
\text { (Herzfrequenzvariabilität) nach } 3 \text { und } 6 \text { Monaten } \\
\text { 5. Trainingsadhärenz nach } 3 \text { und } 6 \text { Monaten }\end{array}$ \\
\hline Sicherheit und Verträglichkeit: & $\begin{array}{l}\text { 1. Klinische Verträglichkeit des körperlichen Trainings } \\
\text { 2. Einzeln sowie kombiniert Morbidität und Mortalität (jeder sowie } \\
\text { kardiovaskulärer Ursache; Tod bzw. Hospitalisation) nach } 3 \\
\text { und } 6 \text { Monaten }\end{array}$ \\
\hline Biometrie: & $\begin{array}{l}\text { Die Analyse der Endpunkte erfolgt durch t-Test für gepaarte } \\
\text { Stichproben (Kohortenanalyse der Trainingsgruppe) sowie durch } \\
\text { Kovarianzanalyse (Gruppenvergleich), ggf. mit Kovariablen. }\end{array}$ \\
\hline Zeitplan: & $\begin{array}{l}\text { Rekrutierung vom 01.01.2007 bis } 31.12 .2007 \text {, bei nicht } \\
\text { ausreichendem Rekrutierungserfolg Verlängerung bis } 30.06 .2008 \\
\text { möglich, Nachbeobachtung nach } 3 \text { und } 6 \text { Monaten. Damit Ende } \\
\text { der Studie spätestens zum } 31.12 .08\end{array}$ \\
\hline
\end{tabular}


TP9A: IKARIUS

\begin{tabular}{|c|c|}
\hline Titel der Studie: & $\begin{array}{l}\text { Die inflammatorische dilatative Kardiomyopathie als Manifestation einer } \\
\text { autoimmunen Diathese }\end{array}$ \\
\hline Kurzbezeichnung der Studie: & Ikarius \\
\hline Indikation: & Dilatative Kardiomyopathie (familiär / nicht familiär) \\
\hline Studienziele: & $\begin{array}{l}\text { Schätzung der Prävalenz familiärer DCM unter DCM-Patienten; } \\
\text { Vergleich familiärer und sporadischer Genese hinsichtlich Prävalenz } \\
\text { inflammatorischer DCM; } \\
\text { Geschlechtervergleich } \\
\text { Fragestellungen zu genetischen Untersuchungen an Vollblut der Patienten: } \\
\text { Gibt es in diesen Familien einen genetischen Link zu } \\
\text { Autoimmunerkrankungen? Gibt es in diesen Familien eine genetische } \\
\text { Prädisposition für Infektionserkrankungen? Gibt es neben einem } \\
\text { genetischen Link bzw. einer genetischen Prädisposition auch eine } \\
\text { veränderte Regulation der Expression dieser Gene? }\end{array}$ \\
\hline Studiendesign: & Kohortenstudie \\
\hline Studienpopulation: & $\begin{array}{l}\text { Einschlusskriterien: } \\
\text { - Linksventrikuläre Ejektionsfraktion }(E F)<45 \% \text { in der Echokardiographie } \\
\text { - Diastolischer Durchmesser des linken Ventrikels (LVEDD) } \geq 117 \% \text { des } \\
\text { erwarteten Wertes (berechnet als Funktion von Alter und Körperoberfläche } \\
\text { nach den Henry Tabellen -Henry-Index- 1980) } \\
\text { ODER } \\
\text { Diastolischer Durchmesser des linken Ventrikels } \geq 112 \% \text { (Henry-Index) } \\
\text { plus AV-Blockierung, Linksschenkelblock oder Vorhofflimmern vor dem } \\
\text { 50. Lebensjahr } \\
\text { - Alter 18-70 Jahre bei Diagnosestellung } \\
\text { - schriftliche Einwilligung des Patienten muß vorliegen } \\
\text { Ausschlusskriterien: } \\
\text { - Koronarstenose (>50\% einer relevanten Koronararterie, } \\
\text { Koronarangiographie erforderlich), } \\
\text { - Arterielle Hypertonie mit Endorganschäden bzw. RR > 140/80 mm Hg } \\
\text { unter antihypertensiver Therapie } \\
\text { - Relevantes Klappenvitium } \\
\text { - mangelnde Kooperationsbereitschaft (Compliance) } \\
\text { - psychische, mentale oder sprachl. Probleme, die die } \\
\text { Einwilligungsfähigkeit und/oder das Verständnis der Fragebögen } \\
\text { beeinflussen könnten } \\
\text { - Teilnahme an einer anderen Studie, die die Teilnahme an der } \\
\text { vorliegenden Verlaufsbeobachtung verbietet. }\end{array}$ \\
\hline Patientenzahl: & $\begin{array}{l}\text { Screeninguntersuchung } 1000 \text { Patienten } \\
\text { Studieneinschluß: } 350 \text { Patienten mit DCM } \\
\text { davon voraussichtlich ca. } 100 \text { Index-Patienten mit genetisch bedingter } \\
\text { DCM (mit entspr. Familienanamnese) } \\
\text { davon ca. } 20 \text { mit inflammatorischer Komponente, } 80 \text { idiopathisch }\end{array}$ \\
\hline Endpunkte: & $\begin{array}{l}\text { Klinisch: Klinische Verlaufsparameter, Echoparameter, kardiale Ereignisse, } \\
\text { familienanamnestische Angaben; } \\
\text { Genetische Marker (Kandidatengene) }\end{array}$ \\
\hline
\end{tabular}


Anhang

\begin{tabular}{|l|l|}
\hline Biometrie: & $\begin{array}{l}\text { Prävalenzschätzung; } \mathrm{Chi}^{2} \text { und loglineare Zusammenhangsanalyse; } \\
\text { ANOVA; Linkage-Analyse }\end{array}$ \\
\hline Zeitplan: & $\begin{array}{l}\text { Je Patient: mindestens } 12 \text { Monate } \\
\text { Studienbezogen : Beginn } 07 / 2004, \text { Rekrutierung bis } 07 / 2007, \\
\text { Nachbeobachtung bis } 07 / 2008\end{array}$ \\
\hline
\end{tabular}


TP9B: HIV \& Herz

\begin{tabular}{|c|c|}
\hline Titel der Studie: & $\begin{array}{l}\text { Chronische Herzinsuffizienz bei HIV-positiven Patienten: Studie zu kardialen } \\
\text { Erkrankungen bei von der HIV-Infektion betroffenen Patienten }\end{array}$ \\
\hline Kurzbezeichnung der Studie: & HIV \& Herz \\
\hline Indikation: & HIV-assoziierte chronische Herzinsuffizienz \\
\hline Primäres Ziel der Studie: & $\begin{array}{l}\text { Erfassung der Inzidenz, Prävalenz sowie der Ätiologie der chronischen } \\
\text { Herzinsuffizienz und deren Verlauf bei HIV-positiven Patienten }\end{array}$ \\
\hline Sekundäre Ziele der Studie: & 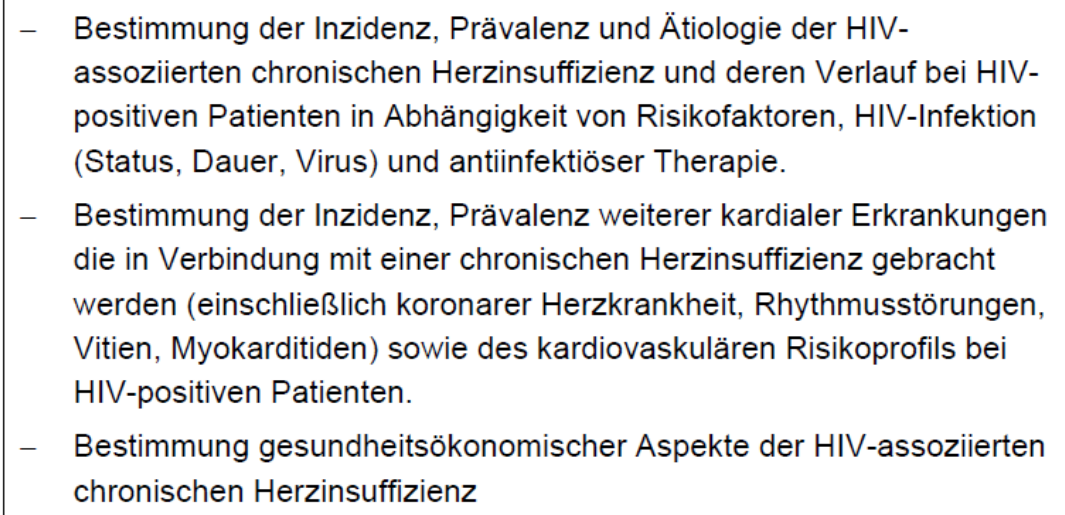 \\
\hline Studiendesign: & epidemiologische, monozentrische Studie \\
\hline Studienpopulation: & $\begin{array}{l}\text { Einschlusskriterien } \\
\text { - bekannte HIV-Infektion } \\
\text { - Alter } \geq 18 \text { Jahre } \\
\text { - schriftliche Einwilligung des Patienten liegt vor } \\
\text { - stabiler Krankheitsverlauf innerhalb der letzten } 4 \text { Wochen } \\
\text { Ausschlusskriterien } \\
\text { - Instabile Herz-Kreislauf Situation } \\
\text { - aktuelle stationäre Behandlung aufgrund anderer Ursache } \\
\text { - Schwangerschaft }\end{array}$ \\
\hline Patientenzahl: & 800 Patienten \\
\hline Therapie: & Kein Therapieschema im Rahmen der Studie vorgesehen. \\
\hline Primärer Endpunkt: & Diagnose der chronischen Herzinsuffizienz \\
\hline Sekundäre Endpunkte: & Kardiale Diagnosen, Komplikationen, Kostenfaktoren, Quality of Life (QoL) \\
\hline Biometrie: & $\begin{array}{l}\text { 95\%-Konfidenzschätzung für Prävalenz und kumulativen Inzidenz durch } \\
\text { approximative Gauß-Statistik; QoL-Verlauf durch MANOVA }\end{array}$ \\
\hline Zeitplan: & $\begin{array}{l}\text { Patientenbezogen: } 24 \text { Monate einschl. Nachbeobachtungsphase } \\
\begin{aligned} \text { Studienbezogen: } & \text { - Beginn: Juli } 2004 \\
& - \text { Rekrutierungsdauer: } 24 \text { Monate } \\
& - \text { vorauss. Abschluss Rekrutierung: Juli } 2006 \\
& - \text { Nachbeobachtungsphase: } 24 \text { Monate } \\
& - \text { vorauss. Abschluss Nachbeob.: Juli } 2008\end{aligned}\end{array}$ \\
\hline
\end{tabular}


TP10: HELPS

\begin{tabular}{|c|c|}
\hline Titel der Studie: & $\begin{array}{l}\text { Heidelberger Studie zur Verlaufsuntersuchung von Lebensqualität und } \\
\text { psychischer Komorbidität bei depressiven Herzinsuffizienz-Patienten } \\
\text { (Heidelberg study of longitudinal evaluation of psychosocial variables in } \\
\text { depressed heart failure patients) }\end{array}$ \\
\hline Kurzbezeichnung der Studie: & HELPS \\
\hline Indikation: & Chronische Herzinsuffizienz \\
\hline Primäres Ziel der Studie: & $\begin{array}{l}\text { Erfassung des Einflusses von Depression auf die Lebensqualität } \\
\text { herzinsuffizienter Patienten über die Zeit }\end{array}$ \\
\hline Sekundäre Ziele der Studie: & $\begin{array}{l}\text { Erfassung des Einflusses von Depression auf die Compliance und die } \\
\text { kognitive Insuffizienz. } \\
\text { Vergleich depressiver und nicht depressiver herzinsuffizienter Patienten } \\
\text { bezüglich der Interaktion psychosozialer und somatischer Variablen zu T1 } \\
\text { (0 Mo.) und T2 (12 Mo.). }\end{array}$ \\
\hline Studiendesign: & Quasiexperimentelle Quer- und Längsschnittstudie. \\
\hline Studienpopulation: & 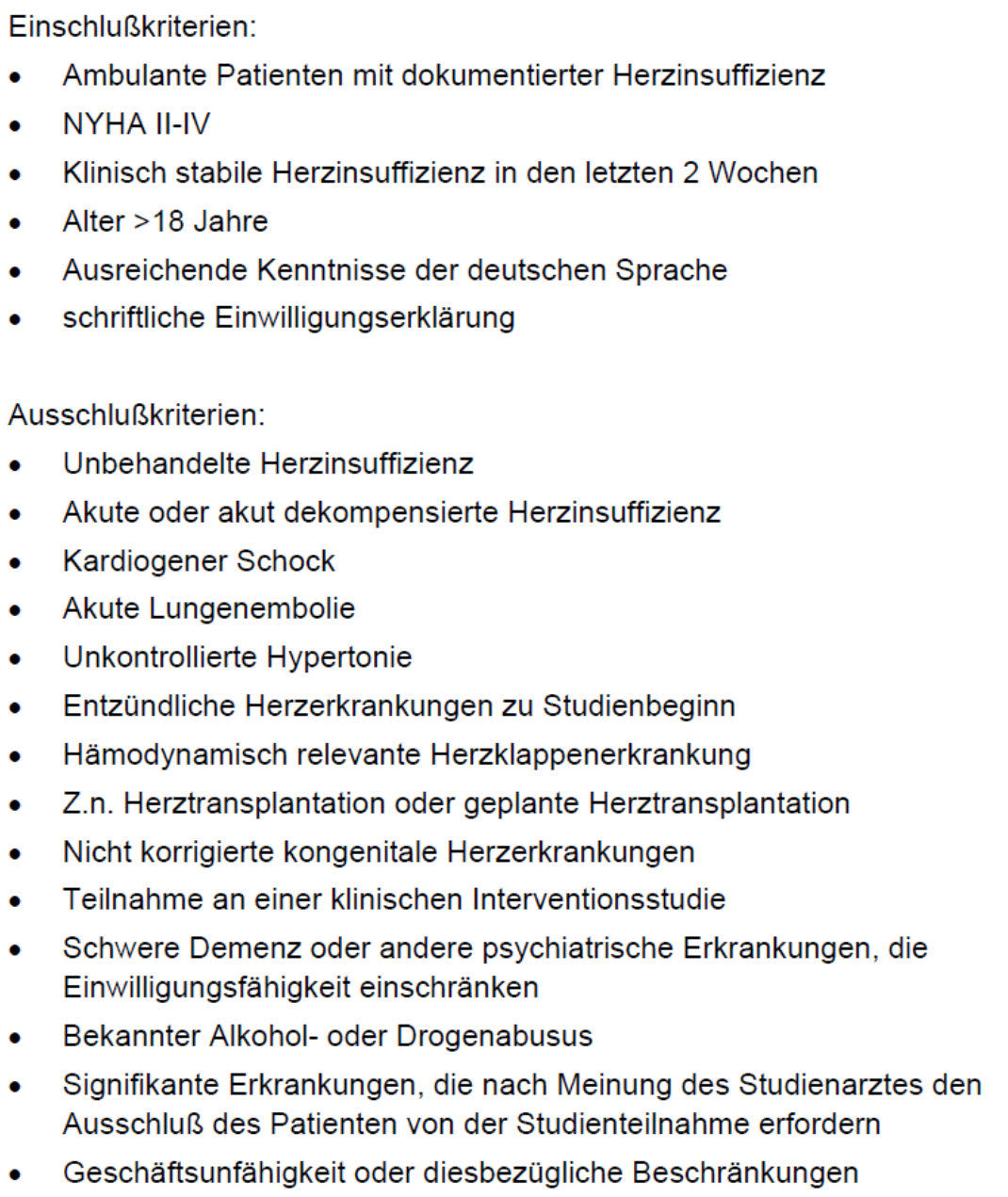 \\
\hline Patientenzahl: & 400 Patienten in den beteiligten Zentren \\
\hline Therapie: & Entfällt \\
\hline Primärer Outcomeparameter: & Lebensqualität \\
\hline Sekundäre & Depressivität, Compliance, Multimorbidität, kognitive Insuffizienz, \\
\hline
\end{tabular}


Anhang

\begin{tabular}{|l|l|}
\hline Outcomeparameter: & $\begin{array}{l}\text { Inanspruchnahmeverhalten, soziale Unterstützung, Versorgungsbedarf und } \\
\text { Inanspruchnahmeverhalten, Angst, körperliches Wohlbefinden, } \\
\text { Rehospitalisierung und Tod, Klinische Ausprägung der Herzinsuffizienz } \\
\text { (definiert am NYHA-Stadium) }\end{array}$ \\
\hline Biometrie: & $\begin{array}{l}\text { Vergleichende parametrische und non-parametrische Tests (u.a. T-Tests, } \\
\text { Varianzanalysen), Partielle Korrelationsanalysen (Pfadanalysen), Bivariate } \\
\text { Korrelationen, Regressionsanalysen }\end{array}$ \\
\hline Zeitplan: & $\begin{array}{l}\text { Ethikvotum: bereits erteilt } \\
\text { Beginn Patienteneinschluss: 01.08.2004 } \\
\text { Ende Patientenbefragung: 31.07.2006 } \\
\text { Zwischenauswertung: ab 31.07.2005 }\end{array}$ \\
\hline
\end{tabular}


TP10: TtT

\begin{tabular}{|c|c|}
\hline Titel der Studie: & $\begin{array}{l}\text { Schulung von Hausärzten zur Verbesserung der Lebensqualität von } \\
\text { Patienten mit chronischer Herzinsuffizienz }\end{array}$ \\
\hline Kurzbezeichnung der Studie: & Train the trainer \\
\hline Indikation: & Chronische (systolische) Herzinsuffizienz \\
\hline Primäres Ziel der Studie: & $\begin{array}{l}\text { Verbesserung der gesundheitsbezogenen Lebensqualität der Patienten: } \\
\text { Vergleich zweier Schulungsansätze (Standard-Schulung vs. Train the } \\
\text { Trainer + Qualitätszirkel) hinsichtlich der Veränderung der } \\
\text { Lebensqualitätsdomäne } \\
\text { „Körperliche Funktionsfähigkeit“ (KF). }\end{array}$ \\
\hline Sekundäre Ziele der Studie: & $\begin{array}{l}\text { Verbesserung der krankheitsspezifischen LQ und anderer Domänen der } \\
\text { LQ, Senkung von Rehospitalisierungsraten, Mortalität, Verbesserung der } \\
\text { Versorgungsqualität der Patienten }\end{array}$ \\
\hline Studiendesign: & 2-armig, kontrolliert, offen, cluster-randomisiert \\
\hline Studienpopulation: & $\begin{array}{l}\text { Einschlusskriterien: } \\
\text { - } \quad \text { Ambulante Patient/Innen } \\
\text { - } \quad \text { Pännliche und weibliche Patient/Innen, Alter } \geq 40 \text { Jahre } \\
\text { biventrikulärer Herzinsuffizienz NYHA II-IV, EF } \leq 40 \% \text {. } \\
\text { Ausschlusskriterien } \\
\text { - } \quad \text { Akute oder akut dekompensierte oder unbehandelte Herzinsuffizienz } \\
\text { - Unkontrollierte Hypertonie } \\
\text { - } \quad \text { Hämodynamisch relevante Herzklappenerkrankung } \\
\text { - } \quad \text { Kongenitale Herzerkrankungen } \\
\text { - } \quad \text { hypertrophe oder restriktive Kardiomyopathie }\end{array}$ \\
\hline Patientenzahl: & $\begin{array}{l}480 \text { Patienten in } 60 \text { Hausarztpraxen, } 30 \text { Praxen mit } 240 \text { Patienten je Arm, } \\
\text { (durchschnittlich acht Patienten/Praxis) }\end{array}$ \\
\hline Therapie: & $\begin{array}{l}\text { Vermittlung von Kompetenzen zur Optimierung der Lebensqualität und } \\
\text { Fähigkeiten zum Selbstmanagement an die Hausärzte und Training in der } \\
\text { Weitergabe dieses Wissens an die Patienten }\end{array}$ \\
\hline Primärer Endpunkt: & $\begin{array}{l}\text { Lebensqualität der Patienten (gesundheitsbezogene LQ-Veränderung, } \\
\text { bezogen auf die Subskala „Körperliche Funktionsfähigkeit“ des SF-36 von } \\
\text { T2 nach T3.) }\end{array}$ \\
\hline Sekundäre Endpunkte: & $\begin{array}{l}\text { zusätzliche Lebensqualitäts-Domänen, krankheitsspezifische LQ, } \\
\text { Versorgungsqualität der Patienten, Schweregrad der Herzinsuffizienz } \\
\text { gemessen an NT-proBNP, Compliance und Depressivität, außerdem } \\
\text { Rehospitalisierung und Mortalität als kombinierter Endpunkt, Evaluation } \\
\text { der Schulungen }\end{array}$ \\
\hline Biometrie: & $\begin{array}{l}\text { Feststellung des Schulungseffektes nach dem Prinzip „intention to treat": } \\
\text { primär Varianzanalyse mit Korrektur für Clustereffekt. Regression für } \\
\text { Kovariableneffekte. }\end{array}$ \\
\hline Zeitplan: & $\begin{array}{l}\text { - } \quad \text { Primäres Ethikvotum: } 28.08 .04 \\
\text { - } \quad \text { Votum des hier vorliegenden Studienprotokolls: } 24.02 .2005 \\
\text { - } \quad \text { Reknendment der Version vom 28.08.04) } \\
\text { - }\end{array}$ \\
\hline
\end{tabular}


Anhang

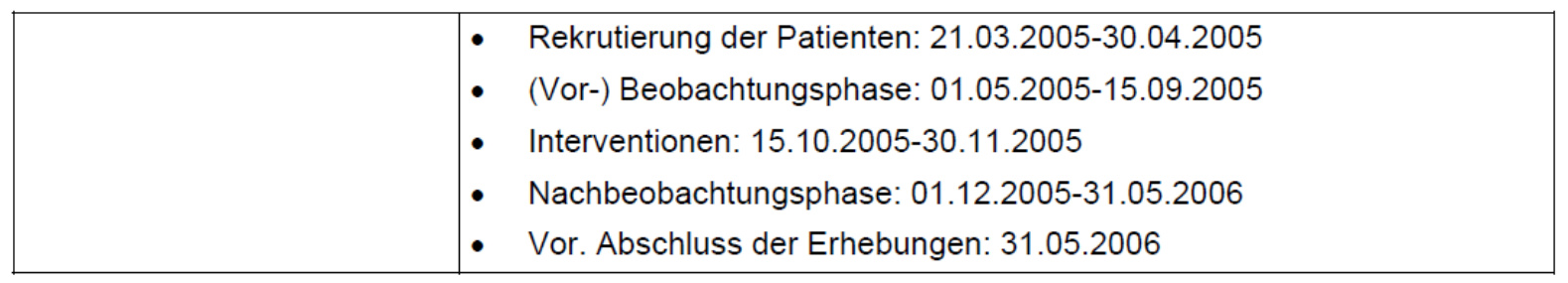


TP12: CIBIS-ELD

\begin{tabular}{|c|c|}
\hline Titel der Studie: & $\begin{array}{l}\text { Vergleich von Bisoprolol mit Carvedilol bei älteren Patienten mit } \\
\text { Herzinsuffizienz }\end{array}$ \\
\hline Kurzbezeichnung: & CIBIS-ELD \\
\hline Indikation: & Chronische Herzinsuffizienz \\
\hline Primäre Fragestellung: & $\begin{array}{l}\text { Gibt es Unterschiede in der Verträglichkeit (s.2.1) der Patienten auf } \beta \text { - } \\
\text { Rezeptorblocker beim Einsatz von Bisoprolol vs. Carvedilol? }\end{array}$ \\
\hline Sekundäre Fragen: & $\begin{array}{l}\text { Prüfung auf Unterschiede hinsichtlich der sekundären Endpunkte; Prüfung } \\
\text { des möglichen Einflusses von Baseline-Daten, Labor, des CYP- } \\
\text { Polymorphismus, der Depressivität und der Compliance auf die Endpunkte }\end{array}$ \\
\hline Studiendesign: & $\begin{array}{l}\text { randomisiert, } 2 \text { Parallelgruppen, doppelblind mit Double-Dummy-Technik, } \\
\text { multizentrisch, Phase III }\end{array}$ \\
\hline Studienpopulation: & 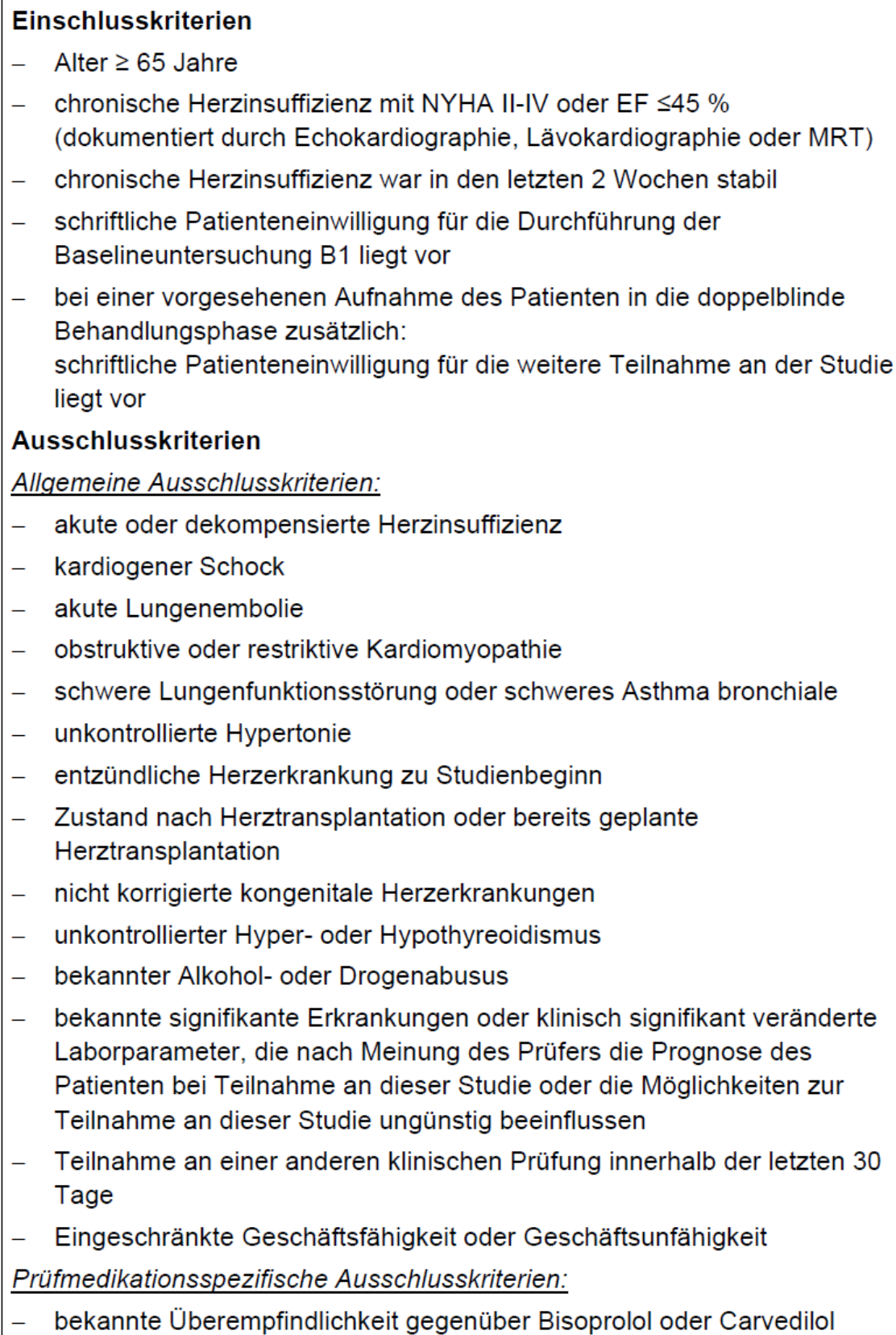 \\
\hline
\end{tabular}




\begin{tabular}{|c|c|}
\hline & 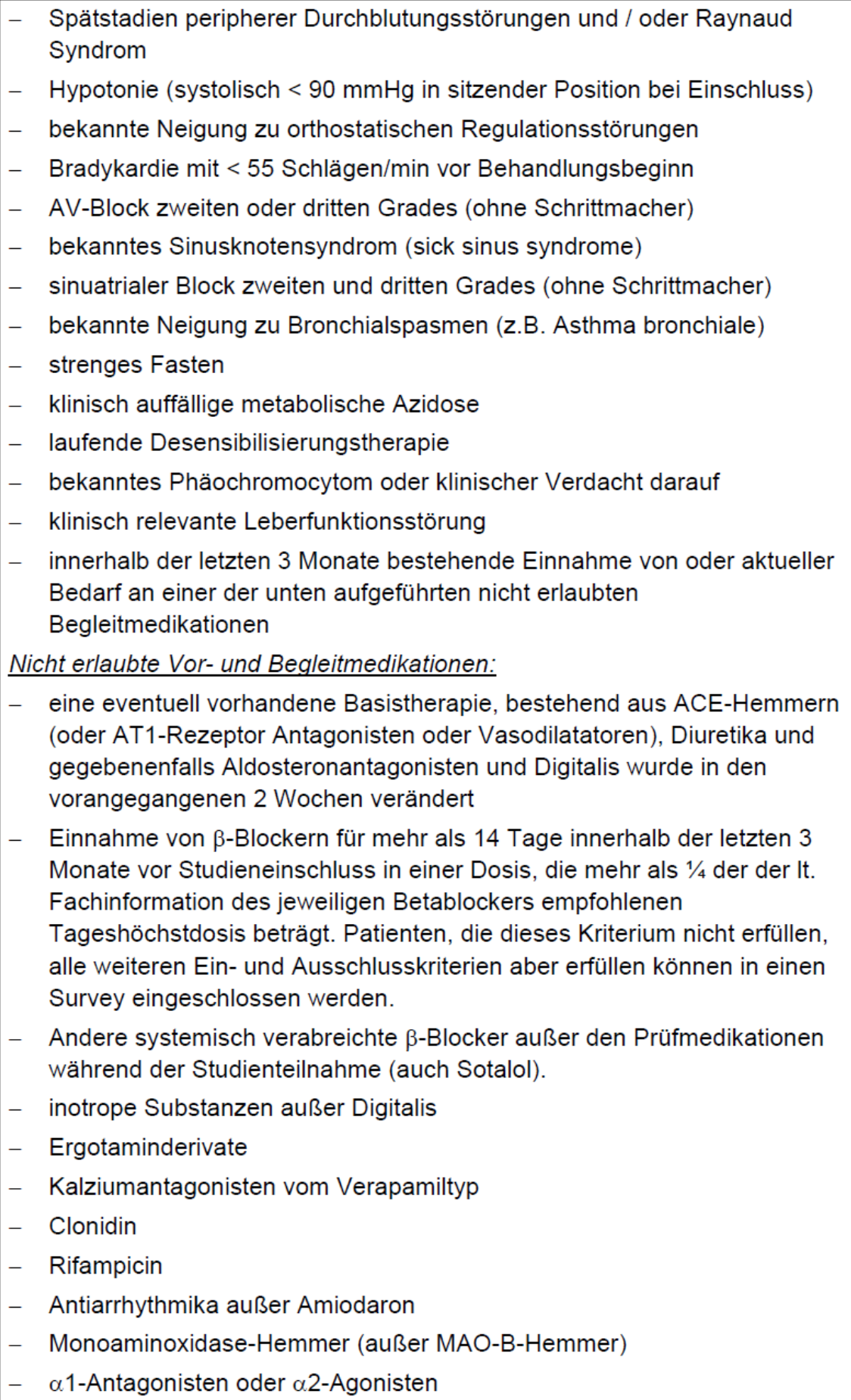 \\
\hline Patientenzahl: & 1040 (520 je Arm) bezogen auf den Titrationsteil \\
\hline Therapie: & $\begin{array}{l}\text { Einstellung auf } \beta \text {-Rezeptorblocker } \\
\text { 1. Betablocker-naive Patienten und Patienten, die bislang maximal } 1 / 8 \text { der It. } \\
\text { Fachinformation des jeweiligen Betablockers empfohlenen Tageshöchstdosis } \\
\text { erhalten haben: Beginnend bei } 1,25 \mathrm{mg} / \mathrm{d} \text { Bisoprolol bzw. } 2 \text { × } 3,125 \mathrm{mg} / \mathrm{d} \\
\text { Carvedilol. } \\
\text { 2. Patienten, die bislang mehr als } 1 / 8 \text {, jedoch maximal } 1 / 4 \text { der It. } \\
\text { Fachinformation des jeweiligen Betablockers empfohlenen Tageshöchstdosis } \\
\text { erhalten haben: Beginnend bei } 2,5 \mathrm{mg} / \mathrm{d} \text { Bisoprolol bzw. } 2 \text { × } 6,25 \mathrm{mg} / \mathrm{d} \\
\text { Carvedilol. }\end{array}$ \\
\hline
\end{tabular}




\begin{tabular}{|c|c|}
\hline & $\begin{array}{l}\text { Dosisverdopplung alle } 14 \text { Tage. Stop, wenn Zieldosis erreicht (Bisoprolol } 10 \\
\mathrm{mg} / \mathrm{d} \text {; Carvedilol } 2 \times 25 \mathrm{mg} / \mathrm{d} \text {, Pat. > } 85 \mathrm{~kg}: 2 \times 50 \mathrm{mg} / \mathrm{d} \text { ) oder weitere } \\
\text { Erhöhung nicht verträglich (ggf. Dosisreduktion). } \\
10 \text { bzw. } 12 \text { Wochen doppelblinde Therapiephase. }\end{array}$ \\
\hline Primärer Endpunkt: & $\begin{array}{l}\text { Der primäre Endpunkt ist die Verträglichkeit (ja/nein) der leitliniengerechten } \\
\text { Maximaldosis der Studienmedikation. }\end{array}$ \\
\hline Sekundäre Endpunkte: & $\begin{array}{l}\text { - Time to Treatment Failure (TTF) } \\
\text { - \%-Dosis } \\
\text { - Zahl der AE/SAE } \\
\text { - Änderung VC, FEV } 1, \text { PEF und MEF }{ }_{50} \text { in der Spirometrie } \\
\text { - Änderung der NYHA-Klasse } \\
\text { - Änderung der Gehstrecke im 6-Minuten-Gehtest } \\
\text { - Änderung der linksventrikulären Funktion (systolisch und diastolisch) } \\
\text { - validierte Skalen der Fragebögen (SF-36, PHQ-D, KWB-16) zur } \\
\text { Lebensqualität } \\
\text { - Änderung der Herzfrequenz } \\
\text { - Änderung der Plasma-NT-proBNP-Konzentration } \\
\text { - Änderung des Plasma-Konzentrationen von Gesamtcholesterin, HDL, LDL, } \\
\text { und Triglyceriden }\end{array}$ \\
\hline Biometrie: & $\begin{array}{l}\text { Primärer Endpunkt: exakter Mann-Whitney U-Test } \\
\text { Sekundäre Endpunkte: Mann-Whitney-Kontrast mit Zensur (erlebte } \\
\text { krankenhausfreie Zeit), Varianzanalyse (metrische Endpunkte), stratifizierte } \\
\text { Odds Ratios nach Cochrane-Mantel-Haenszel mit Breslow-Day- } \\
\text { Homogenitätstest (binäre Endpunkte) } \\
\text { Explorativ: multiple Regressionsanalysen }\end{array}$ \\
\hline Zeitplan: & $\begin{array}{l}\text { je Patient: } 10 \text { - } 12 \text { Wochen } \\
\text { Studienbezogen: Beginn 04/2005, Rekrutierung bis } 03 / 2008 \text {, } \\
\text { Nachbeobachtung bis } 06 / 2008\end{array}$ \\
\hline
\end{tabular}




\section{TP13: ACVB-Out}

\begin{tabular}{|c|c|}
\hline Titel der Studie: & $\begin{array}{l}\text { Geschlechtsspezifische Unterschiede in der Bedeutung von } \\
\text { Herzinsuffizienz, Hormonstatus und psychosozialen Faktoren für } \\
\text { die Prognose nach aortocoronarer Bypass-Operation }\end{array}$ \\
\hline Kurzbezeichnung: & ACVB-Outcome-Studie \\
\hline Indikation: & $\begin{array}{l}\text { Koronare Herzerkrankung mit Indikation zu operativer } \\
\text { Revaskularisierung }\end{array}$ \\
\hline Primäres Ziel der Studie: & $\begin{array}{l}\text { Determinierung geschlechtsspezifischer Unterschiede von } \\
\text { Einflußfaktoren und deren Bedeutung für den Verlauf nach ACVB } \\
\text { Operation. }\end{array}$ \\
\hline Sekundäre Ziele der Studie: & $\begin{array}{l}\text { Untersuchung des Remodelling, des Einflusses des } \\
\text { Revaskularisierungsgrades auf Geschlechterunter-schiede und } \\
\text { des Einflusses des Hormonstatus bei Frauen auf das klinische } \\
\text { Behandlungsergebnis }\end{array}$ \\
\hline Studiendesign: & $\begin{array}{l}\text { Prospektive oligozentrische Quer- und Längsschnitts- } \\
\text { Kohortenstudie. }\end{array}$ \\
\hline Studienpopulation: & $\begin{array}{l}\text { Einschlusskriterien } \\
-\quad \text { Patienten mit koronarer Herzerkrankung, die sich einer ACVB- } \\
\quad \text { Operation unterziehen, mit und ohne Zeichen einer } \\
\quad \text { Herzinsuffizienz; } \\
-\quad \text { Alter ab } 18 \text { Jahre; } \\
-\quad \text { schriftliche Einwilligung } \\
\text { Ausschlußkriterien: } \\
-\quad \text { fehlende Einwilligungsfähigkeit; } \\
-\quad \text { fehlende Auskunftsfähigkeit zum Wohlbefinden } \\
\end{array}$ \\
\hline Patientenzahl: & $\begin{array}{l}2000 \text { Patienten, Frauen und Männer im Verhältnis 1:2 } \\
\text { (entsprechend der klinischen Population) }\end{array}$ \\
\hline Therapie: & Aortocoronare Bypass-Operation \\
\hline Primäre Endpunkte: & $\begin{array}{l}\text { 1. Gesamt-Mortalität und Morbidität innerhalb des ersten Jahres } \\
\text { nach Operation; } 2 \text {. Verschlechterung ds Wohlbefindens }\end{array}$ \\
\hline Sekundäre Endpunkte: & Komponenten der primären Endpunkte; Remodelling \\
\hline Biometrie: & $\begin{array}{l}\text { primär: 1. Kaplan-Meier-Analyse; 2. approx. Gauß-Test } \\
\text { sekundär: Varianzanalyse, Regressionsmodelle }\end{array}$ \\
\hline Zeitplan: & $\begin{array}{l}\text { Rekrutierung } 1 \text { Jahr, Beobachtung je Patient } 1 \text { Jahr; } \\
\text { Beginn der Rekrutierung ca. November 2004, } \\
\text { voraussichtlicher Abschluss November } 2006\end{array}$ \\
\hline
\end{tabular}




\section{TP15: INH}

\begin{tabular}{|c|c|}
\hline Titel der Studie: & $\begin{array}{l}\text { Krankheitsmanifestation und Krankheitsmanagement bei chronischer } \\
\text { Herzinsuffizienz - Eine randomisierte kontrollierte Follow-up Studie }\end{array}$ \\
\hline Kurzbezeichnung: & Randomisierte INH-Studie \\
\hline Indikation: & Chronische Herzinsuffizienz \\
\hline Primäres Ziel der Studie: & $\begin{array}{l}\text { Prüfung der Effektivität und Effizienz eines umfassenden } \\
\text { Krankheitsmanagements, bestehend aus internistisch-kardiologischer } \\
\text { Betreuung sowie psychoedukativer Intervention (Patientenschulung) zur } \\
\text { Förderung von Selbstmanagement und Empowerment }\end{array}$ \\
\hline Sekundäre Ziele: & $\begin{array}{l}\text { Identifikation von Kovariaten des Outcomes. Quantifizierung der Guideline- } \\
\text { konformen Medikation. Kostenevaluation. }\end{array}$ \\
\hline Studiendesign: & Zweiarmig, parallel, randomisiert, offen, monozentrisch \\
\hline Studienpopulation: & $\begin{array}{l}\text { Einschlusskriterien } \\
\text { - } \text { Volljährigkeit } \\
\text { - } \quad \text { Schriftliche Einverständniserklärung } \\
\text { - } \text { Herzinsuffizienz während eines stationären Aufenthaltes dokumentiert } \\
\text { durch eingeschränkte linksventrikuläre Ejektionsfraktion (EF) } \leq 40 \% \\
\text { (dokumentiert im Echokardiogramm durch biplane EF-Messung oder in } \\
\text { der Ventrikulographie i.R. der Herzkatheteruntersuchung oder bei der } \\
\text { Radionuklidventrikulographie) und klinische Zeichen/Symptome der } \\
\text { Herzinsuffizienz (periphere Ödeme, Lungenödem, pulmonale feuchte } \\
\text { Rasselgeräusche, Lungenstau im Röntgen-Thorax, Einflussstauung, } \\
\text { Belastungsdyspnoe, Leistungsschwäche) } \\
\text { Ausschlußkriterien: } \\
\text { - Nicht ausreichende mentale oder sprachliche oder körperliche Fähigkeit, } \\
\text { oder aus logistischen Gründen fehlende Möglichkeit (z.b. fehlender } \\
\text { Telefonanschluss), an einem ambulanten Betreuungsprogramm mit } \\
\text { Schulungselementen und Telefon-Monitoring teilzunehmen (falls die } \\
\text { Studien-relevante Information über eine den Patienten regelmäßig } \\
\text { betreuende Person eingeholt werden kann und falls dieser die Schulungs- } \\
\text { relevanten Elemente dem Patienten weitervermitteln kann, kann der } \\
\text { Patient in die Studie aufgenommen werden). }\end{array}$ \\
\hline Patientenzahl: & 700 (zwei Studienarme mit je 350 Patienten pro Arm) \\
\hline Intervention: & $\begin{array}{l}\text { Intensivierte Patientenbetreuung im Vergleich zu „Usual Care“, } \\
\text { gekennzeichnet durch } \\
\text { a) Telefonisches Monitoring patientenbezogener Symptome nach einem } \\
\text { prädefinierten Algorithmus abhängig vom Schweregrad der } \\
\text { Herzinsuffizienz } \\
\text { b) Praktische und telefonische patientenzentrierte Schulung bezüglich } \\
\text { typischer Krankheitsmanifestationen und Begleitprobleme bei chronischer } \\
\text { Herzinsuffizienz, z.B. Symptome, Medikation, Ernährung, psychische } \\
\text { Dimensionen }\end{array}$ \\
\hline Primärer Endpunkt: & Tod oder Hospitalisierung jeder Ursache (kombinierter Endpunkt) \\
\hline Sekundäre Endpunkte: & $\begin{array}{l}\text { Komponenten des kombinierten primären Endpunkts einzeln; } \\
\text { kardiovaskulärer Tod; Hospitalisierung aus kardiovaskulärer Indikation; } \\
\text { Prozentsatz Guideline-konform therapierter Patienten; Kosten; Lebensqualität }\end{array}$ \\
\hline Biometrie: & Der primäre Endpunkt wird durch eine Time-to-event-Analyse (Kaplan-Meier) \\
\hline
\end{tabular}




\begin{tabular}{|c|c|c|}
\hline & ausgewertet. & \\
\hline \multirow[t]{2}{*}{ Zeitplan: } & Patientenbezogen: Telefoninterventio & \\
\hline & $\begin{array}{l}\text { Studienbezogen: Beginn 12/200 } \\
\text { voraussichtlicher Abschluss 10/2005. }\end{array}$ & Monate; \\
\hline
\end{tabular}

\subsection{Beispielhafte Darstellung von Screenshot- und Outputdokumenten}

\subsubsection{Ordinale Regressionsanalyse: Screenshot-Dokument am Beispiel von Diabetes mellitus}

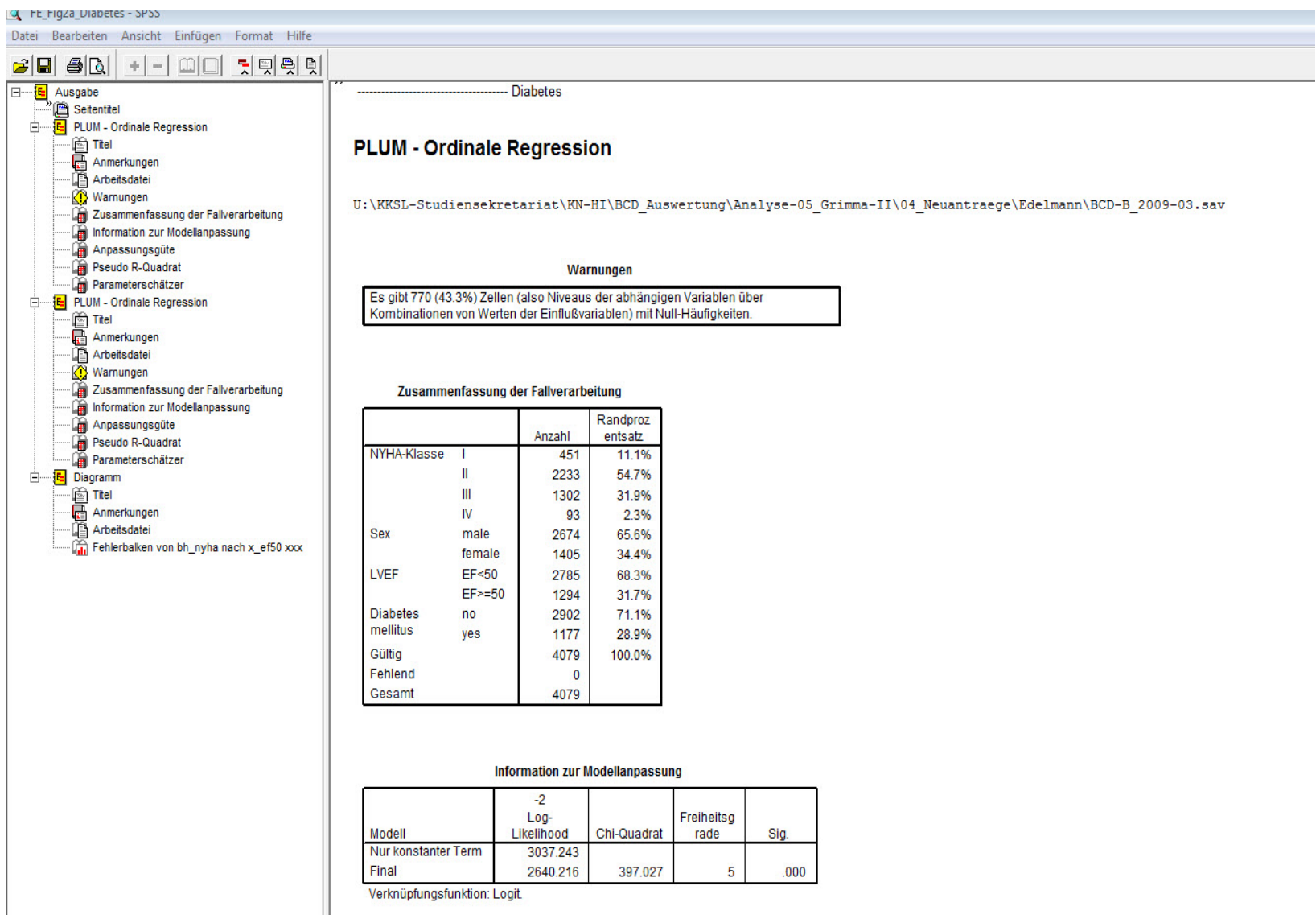




\subsubsection{Ordinale Regressionsanalyse: SPSS-Outputdokument am Beispiel von Diabetes mellitus}

- Diabetes

\section{PLUM - Ordinale Regression \\ $\mathrm{U}: \backslash \mathrm{KKSL}-$ Studiensekretariat $\backslash \mathrm{KN}$-HI $\backslash$ BCD_Auswertung $\backslash$ Analyse-05_Grimma-II $\backslash 04$ Neuantraege $\backslash$ Edelmann $\backslash$ BCD-B_2009-03.sav \\ Warnungen}

Es gibt 770 (43.3\%) Zellen (also Niveaus der abhängigen Variablen über

Kombinationen von Werten der Einflußvariablen) mit Null-Häufigkeiten.

Zusammenfassung der Fallverarbeitung

\begin{tabular}{|ll|r|r|}
\hline \multicolumn{2}{|l|}{} & Anzahl & $\begin{array}{c}\text { Randproz } \\
\text { entsatz }\end{array}$ \\
\hline NYHA-Klasse & I & 451 & $11.1 \%$ \\
& II & 2233 & $54.7 \%$ \\
& III & 1302 & $31.9 \%$ \\
Sex & IV & 93 & $2.3 \%$ \\
& male & 2674 & $65.6 \%$ \\
LVEF & female & 1405 & $34.4 \%$ \\
& EF $<50$ & 2785 & $68.3 \%$ \\
Diabetes & EF>=50 & 1294 & $31.7 \%$ \\
mellitus & no & 2902 & $71.1 \%$ \\
Gültig & yes & 1177 & $28.9 \%$ \\
Fehlend & & 4079 & $100.0 \%$ \\
Gesamt & & 0 & \\
\hline
\end{tabular}

Information zur Modellanpassung

\begin{tabular}{|l|r|r|r|r|}
\hline Modell & \multicolumn{1}{|c|}{$\begin{array}{c}-2 \\
\text { Log-Likelihood }\end{array}$} & Chi-Quadrat & $\begin{array}{c}\text { Freiheitsgr } \\
\text { ade }\end{array}$ & Sig. \\
\hline $\begin{array}{l}\text { Nur konstanter Term } \\
\text { Final }\end{array}$ & $\begin{array}{l}3037.243 \\
2640.216\end{array}$ & 397.027 & 5 & .000 \\
\hline
\end{tabular}

Verknüpfungsfunktion: Logit.

Anpassungsgüte

\begin{tabular}{|l|r|r|r|}
\hline & Chi-Quadrat & $\begin{array}{c}\text { Freiheitsgr } \\
\text { ade }\end{array}$ & \multicolumn{1}{c|}{ Sig. } \\
\hline Pearson & 1636.199 & 1327 & .000 \\
Abweichung & 1332.502 & 1327 & .452 \\
\hline
\end{tabular}

Verknüpfungsfunktion: Logit.

Pseudo R-Quadrat

\begin{tabular}{|l|r|}
\hline Cox und Snell & .093 \\
Nagelkerke & .106 \\
McFadden & .048 \\
\hline
\end{tabular}

Verknüpfungsfunktion: Logit. 
Parameterschätzer

\begin{tabular}{|c|c|c|c|c|c|c|}
\hline & & Schätzer & $\begin{array}{c}\text { Standardf } \\
\text { ehler }\end{array}$ & Wald & $\begin{array}{c}\text { Freiheitsgr } \\
\text { ade }\end{array}$ & Sig. \\
\hline \multirow[t]{3}{*}{ Schwelle } & [bh_nyha = 1] & -1.589 & .159 & 99.444 & 1 & .000 \\
\hline & [bh_nyha = 2] & .812 & .115 & 49.533 & 1 & .000 \\
\hline & [bh_nyha = 3] & 3.382 & .293 & 132.930 & 1 & .000 \\
\hline \multirow[t]{10}{*}{ Lage } & [sex=1] & -.453 & .065 & 48.267 & 1 & .000 \\
\hline & [sex=2] & $0^{\mathrm{a}}$ & & & 0 & \\
\hline & [x_ef50=.00] & 1.077 & .137 & 61.939 & 1 & .000 \\
\hline & [x_ef50=1.00] & $0^{\mathrm{a}}$ & & & 0 & \\
\hline & {$[x x x=.00]$} & -.262 & .106 & 6.089 & 1 & .014 \\
\hline & {$[x x x=1.00]$} & $0^{\mathrm{a}}$ & & & 0 & \\
\hline & [x_ef50=.00] * [xxx=.00] & -.033 & .122 & .072 & 1 & .789 \\
\hline & [x_ef50=.00] * [xxx=1.00] & $0^{\mathrm{a}}$ & & . & 0 & \\
\hline & {$\left[x \_ \text {ef50 }=1.00\right]^{*}[x x x=.00]$} & & . & . & 0 & \\
\hline & $\begin{array}{l}{\left[x \_ \text {ef50 }=1.00\right] \text { * }[x x x=1 .} \\
00]\end{array}$ & & . & - & 0 & \\
\hline Skala & bs_age & -.003 & .001 & 7.225 & 1 & .007 \\
\hline
\end{tabular}

Verknüpfungsfunktion: Logit.

Parameterschätzer

\begin{tabular}{|c|c|c|c|}
\hline & & \multicolumn{2}{|c|}{ Konfidenzintervall 95\% } \\
\hline & & Untergrenze & Obergrenze \\
\hline \multirow[t]{3}{*}{ Schwelle } & [bh_nyha = 1] & -1.902 & -1.277 \\
\hline & [bh_nyha = 2] & .586 & 1.038 \\
\hline & [bh_nyha = 3] & 2.807 & 3.957 \\
\hline \multirow[t]{9}{*}{ Lage } & [sex=1] & -.580 & -.325 \\
\hline & [sex=2] & & \\
\hline & [x_ef50=.00] & .809 & 1.345 \\
\hline & [x_ef50=1.00] & & \\
\hline & $\begin{array}{l}{[x x x=.00]} \\
{[x x x=1.00]}\end{array}$ & -.470 & -.054 \\
\hline & [x_ef50 $=.00]]^{*}[\mathrm{xxx}=.00]$ & -.272 & .207 \\
\hline & [x_ef50 $=.00]$ * $[x x x=1.00]$ & $\cdot$ & \\
\hline & {$\left[x \_ \text {ef50 }=1.00\right]^{*}[x x x=.00]$} & . & \\
\hline & $\begin{array}{l}{\left[x \_ \text {ef50=1.00] * }[x x x=1 .\right.} \\
00]\end{array}$ & & \\
\hline Skala & bs_age & -.006 & -.001 \\
\hline
\end{tabular}

Verknüpfungsfunktion: Logit.

a. Dieser Parameter wird auf Null gesetzt, weil er redundant ist.

\section{PLUM - Ordinale Regression}

$\mathrm{U}: \backslash$ KKSL-Studiensekretariat $\backslash \mathrm{KN}-\mathrm{HI} \backslash$ BCD_Auswertung $\backslash$ Analyse-05_Grimma-II $\backslash 04$ _Neuantraege $\backslash$ Edelmann $\backslash$ BCD-B_2009-03.sav

\section{Warnungen}

Es gibt 770 (43.3\%) Zellen (also Niveaus der abhängigen Variablen über

Kombinationen von Werten der Einflußvariablen) mit Null-Häufigkeiten. 
Diabetes

Zusammenfassung der Fallverarbeitung

\begin{tabular}{|ll|r|r|}
\hline & & Anzahl & $\begin{array}{c}\text { Randproz } \\
\text { entsatz }\end{array}$ \\
\hline NYHA-Klasse & I & 451 & $11.1 \%$ \\
& II & 2233 & $54.7 \%$ \\
& III & 1302 & $31.9 \%$ \\
Sex & IV & 93 & $2.3 \%$ \\
& male & 2674 & $65.6 \%$ \\
LVEF & female & 1405 & $34.4 \%$ \\
& EF $<50$ & 2785 & $68.3 \%$ \\
Diabetes & EF>=50 & 1294 & $31.7 \%$ \\
mellitus & no & 2902 & $71.1 \%$ \\
Gültig & yes & 1177 & $28.9 \%$ \\
Fehlend & & 4079 & $100.0 \%$ \\
Gesamt & & 0 & \\
\hline
\end{tabular}

Information zur Modellanpassung

\begin{tabular}{|l|r|r|r|r|}
\hline Modell & \multicolumn{1}{|c|}{$\begin{array}{c}\text {-2 } \\
\text { Log-Likelihood }\end{array}$} & Chi-Quadrat & $\begin{array}{c}\text { Freiheitsgr } \\
\text { ade }\end{array}$ & \multicolumn{1}{c|}{ Sig. } \\
\hline Nur konstanter Term & 3037.243 & & & \\
Final & 2535.927 & 501.316 & 4 & .000 \\
\hline
\end{tabular}

Verknüpfungsfunktion: Logit.

\section{Anpassungsgüte}

\begin{tabular}{|l|r|r|r|}
\hline & Chi-Quadrat & $\begin{array}{c}\text { Freiheitsgr } \\
\text { ade }\end{array}$ & \multicolumn{1}{c|}{ Sig. } \\
\hline Pearson & 1476.423 & 1328 & .003 \\
Abweichung & 1228.213 & 1328 & .976 \\
\hline
\end{tabular}

Verknüpfungsfunktion: Logit.

\section{Pseudo R-Quadrat}

\begin{tabular}{|l|l|}
\hline Cox und Snell & .116 \\
Nagelkerke & .133 \\
McFadden & .060 \\
\hline
\end{tabular}

Verknüpfungsfunktion: Logit 


\section{Parameterschätzer}

\begin{tabular}{|c|c|c|c|c|c|c|}
\hline & & Schätzer & $\begin{array}{l}\text { Standardf } \\
\text { ehler }\end{array}$ & Wald & $\begin{array}{c}\text { Freiheitsgr } \\
\text { ade }\end{array}$ & Sig. \\
\hline \multirow[t]{3}{*}{ Schwelle } & [bh_nyha = 1] & -.125 & .193 & .419 & 1 & .517 \\
\hline & [bh nyha $=2$ ] & 2.897 & .199 & 211.335 & 1 & .000 \\
\hline & [bh_nyha $=3$ ] & 6.131 & .227 & 731.737 & 1 & .000 \\
\hline \multirow[t]{7}{*}{ Lage } & bs_age & .026 & .002 & 112.953 & 1 & .000 \\
\hline & [sex=1] & -.456 & .069 & 43.874 & 1 & .000 \\
\hline & [sex $=2]$ & $0^{\mathrm{a}}$ & & & 0 & \\
\hline & [x_ef50=.00] & 1.401 & .074 & 355.899 & 1 & .000 \\
\hline & [x_ef50=1.00] & $0^{\mathrm{a}}$ & & & 0 & \\
\hline & {$[x x x=.00]$} & -.235 & .069 & 11.547 & 1 & .001 \\
\hline & {$[x x x=1.00]$} & $0^{\mathrm{a}}$ & & & 0 & \\
\hline
\end{tabular}

Verknüpfungsfunktion: Logit.

\section{Parameterschätzer}

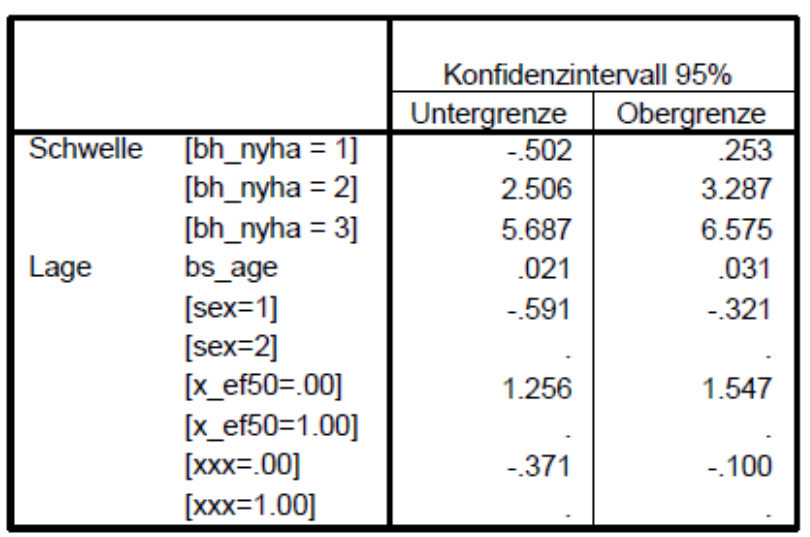

Verknüpfungsfunktion: Logit.

a. Dieser Parameter wird auf Null gesetzt, weil er redundant ist.

\section{Diagramm}

$\mathrm{U}: \backslash \mathrm{KKSL}$-Studiensekretariat $\backslash \mathrm{KN}-\mathrm{HI} \backslash \mathrm{BCD}$ _Auswertung $\backslash$ Analyse-05_Grimma-II $\backslash 04$ Neuantraege $\backslash$ Edelmann $\backslash$ BCD-B_2009-03.sav 

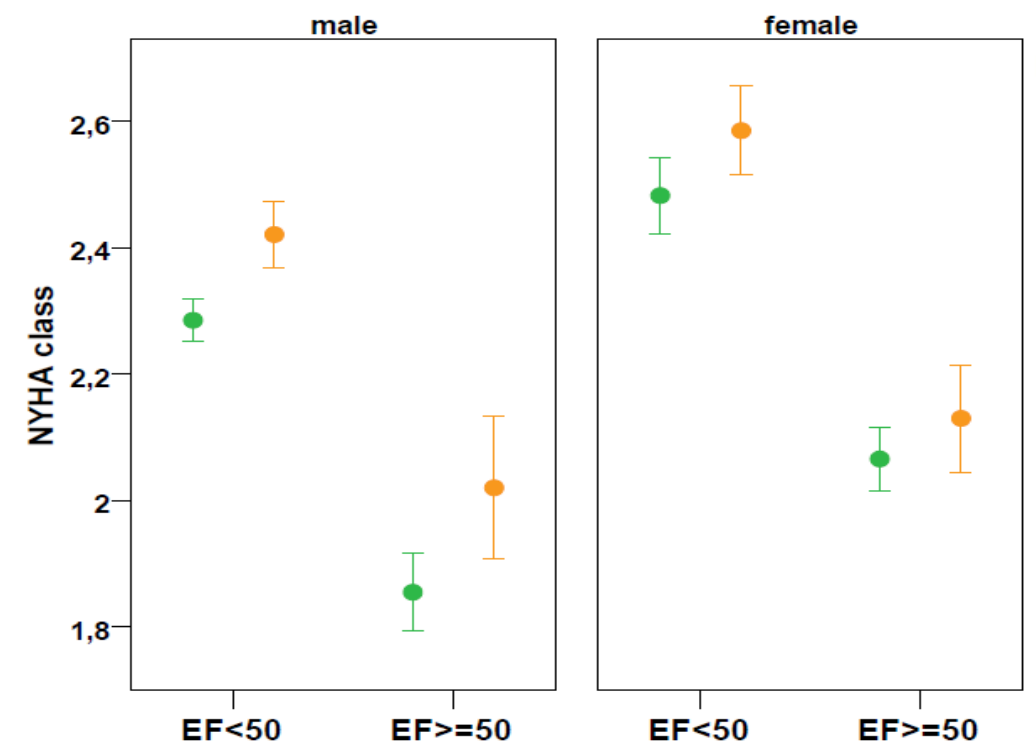

Diabetes mellitus

I no

I yes

OR $=1.26$

Cl:11-1.45

$\mathrm{P}<0.001$

No interaction with LVEF $(P=0.79)$

\subsubsection{Multivariate Analysen: Screenshot- und Outputdokument}

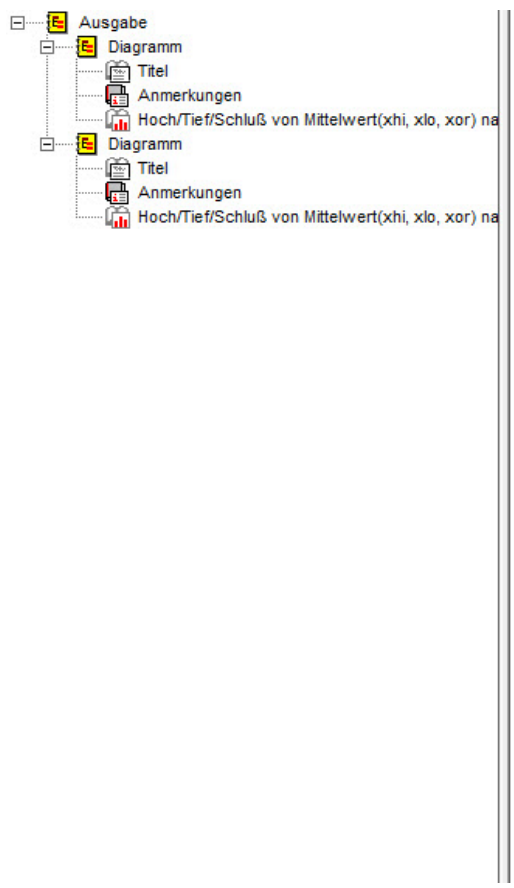

Diagramm

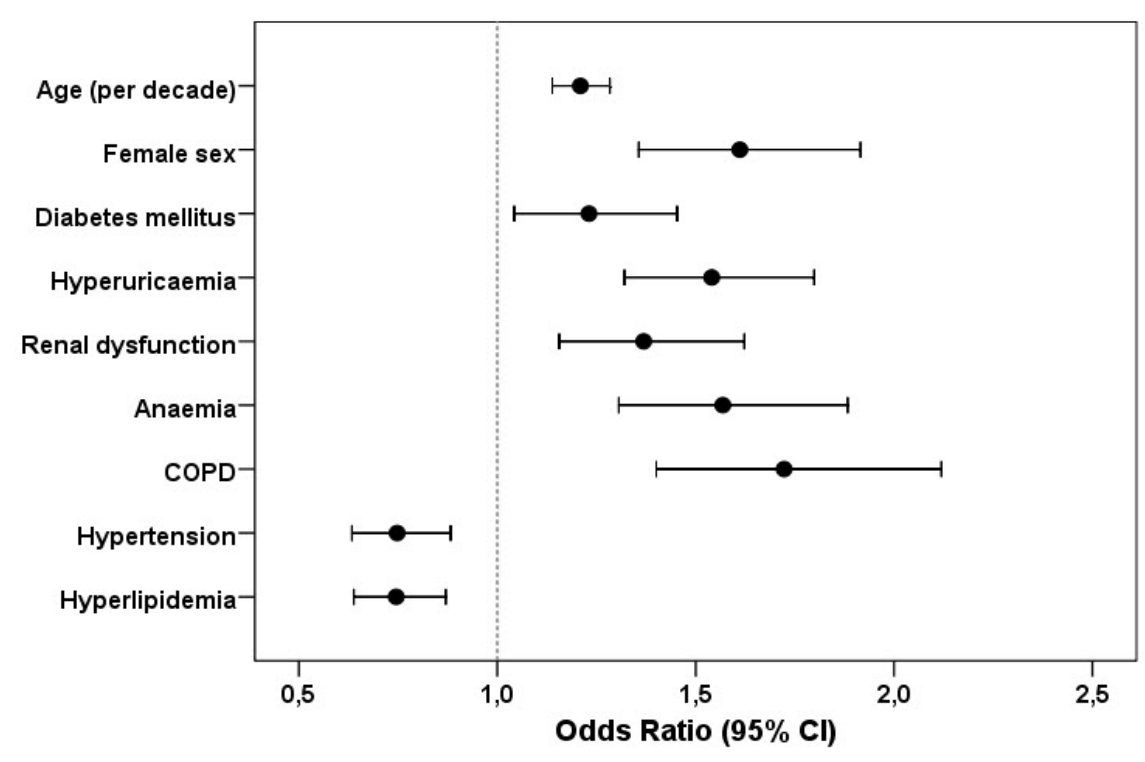


Diagramm

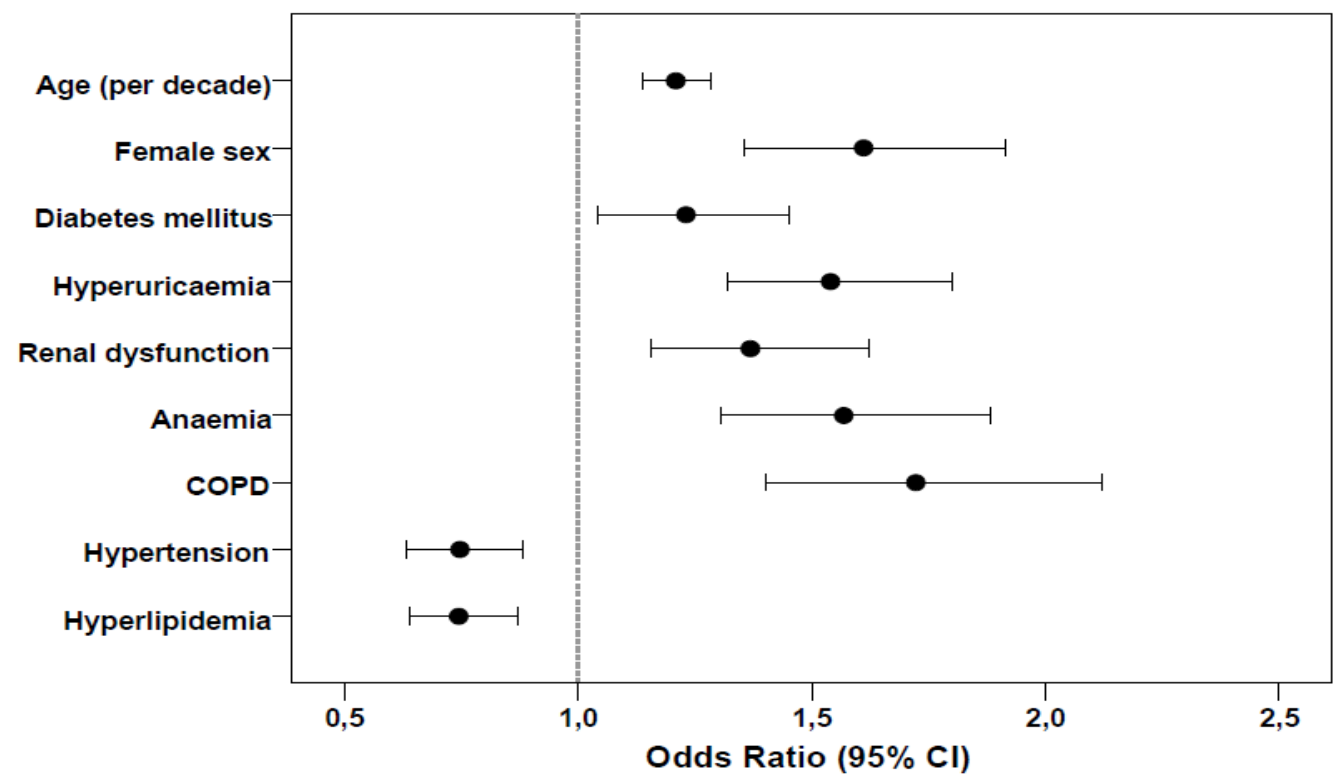

Diagramm

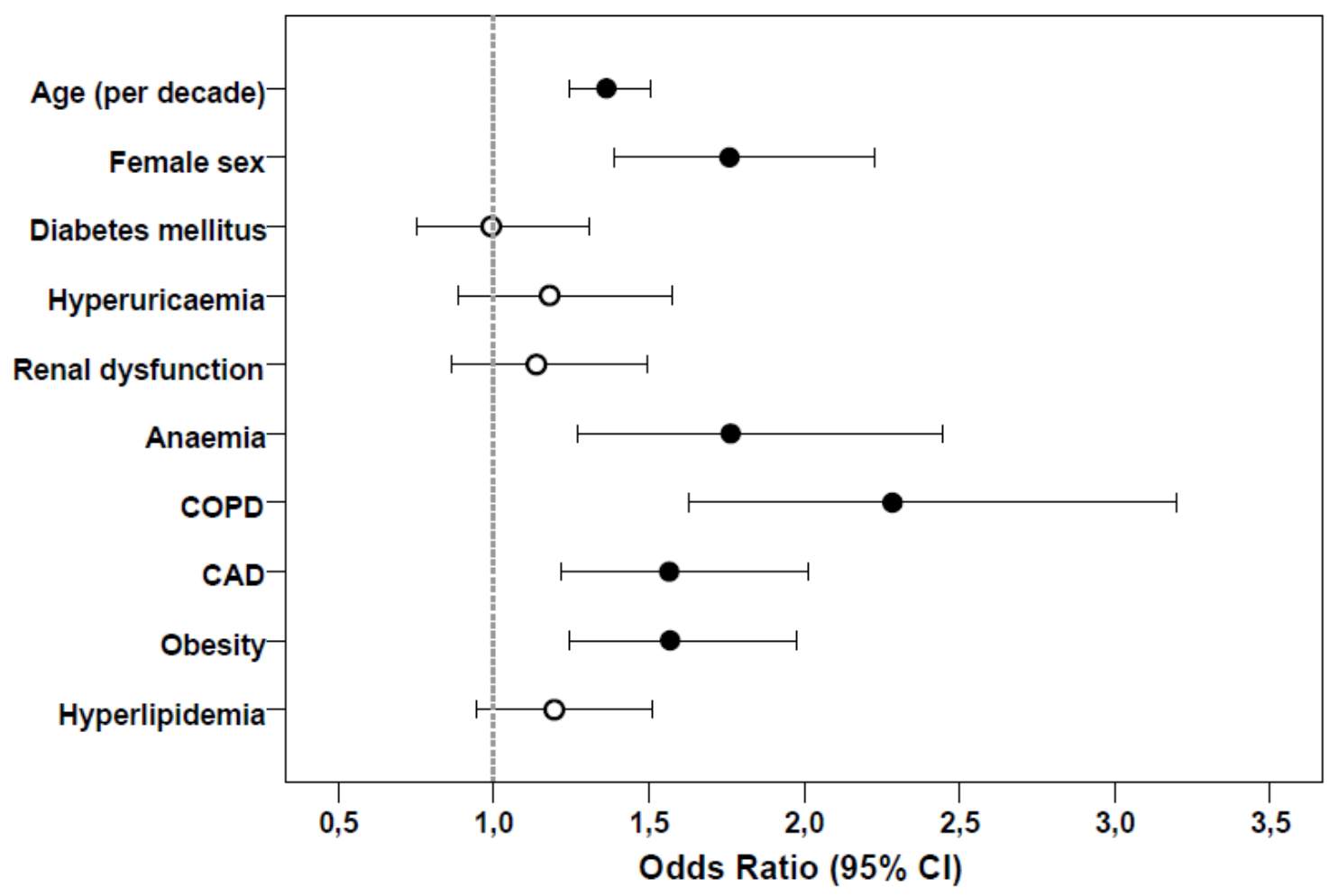




\section{Literaturverzeichnis}

Ahmed A, Rich MW, Fleg JL, Zile MR, Young JB, Kitzman DW, Love TE, Aronow WS, Adams KF Jr, Gheorghiade M (2006): Effects of digoxin on morbidity and mortality in diastolic heart failure: the ancillary digitalis investigation group trial. Circulation $\underline{114}, 397-403$

Ahmed A, Pitt B, Rahimtoola SH, Waagstein F, White M, Love TE, Braunwald E (2008): Effects of digoxin at low serum concentrations on mortality and hospitalization in heart failure: a propensity-matched study of the DIG trial. Int J Cardiol $\underline{123}, 138-46$

Alpert MA, Terry BE, Mulekar M, Cohen MV, Massey CV, Fan TM, Panayiotou H, Mukerji V (1997): Cardiac morphology and left ventricular function in normotensive morbidly obese patients with and without congestive heart failure, and effect of weight loss. Am J Cardiol $\underline{80}$, $736-40$

Anand IS (2008): Heart failure and anemia: mechanisms and pathophysiology. Heart Fail Rev $\underline{13}, 379-86$

Angermann CE (2009): Comorbidites in heart failure: a key issue. Eur J of Heart Fail Suppl $\underline{8}$, i5-i10

Artham SM, Lavie CJ, Milani RV, Ventura HO (2008): The obesity paradox: impact of obesity on the prevalence and prognosis of cardiovascular diseases. Postgrad Med $\underline{120}, 34-41$

Beriault K, Carpentier AC, Gagnon C, Ménard J, Baillargeon JP, Ardilouze JL, Langlois MF (2009): Reproducibility of the 6-minute walk test in obese adults. Int J Sports Med $\underline{30}, 725-7$

Besarab A, Soman S (2005): Anemia management in chronic heart failure: lessons learnt from chronic kidney disease. Kidney Blood Press Res 료, 363-71

Bhatia RS, Tu JV, Lee DS, Austin PC, Fang J, Haouzi A, Gong Y, Liu PP (2006): Outcome of heart failure with preserved ejection fraction in a population-based study. N Engl J Med $\underline{355}$, $260-9$

Boudestein LC, Rutten FH, Cramer MJ, Lammers JW, Hoes AW (2009): The impact of concurrent heart failure on prognosis in patients with chronic obstructive pulmonary disease. Eur J Heart Fail 11, 1182-8 
Bruch C, Rothenburger M, Gotzmann M, Wichter T, Scheld HH, Breithardt G, Gradaus R (2007): Chronic kidney disease in patients with chronic heart failure--impact on intracardiac conduction, diastolic function and prognosis. Int J Cardiol $\underline{118}, 375-80$

Bursi F, Weston SA, Redfield MM, Jacobsen SJ, Pakhomov S, Nkomo VT, Meverden RA, Roger VL (2006): Systolic and diastolic heart failure in the community. JAMA 296, 2209-16

Captopril Multicenter Research Group (1983): A placebo-controlled trial of captopril in refractory chronic congestive heart failure. J Am Coll Cardiol 2, 755-763

Caroci Ade S, Lareau SC (2004): Descriptors of dyspnea by patients with chronic obstructive pulmonary disease versus congestive heart failure. Heart Lung $\underline{33}, 102-10$

Cleland JG, Tendera M, Adamus J, Freemantle N, Gray CS, Lye M, O'Mahony D, Polonski L, Taylor J (1999): Perindopril for elderly people with chronic heart failure: the PEP-CHF study. The PEP investigators. Eur J Heart Fail $\underline{1}$, 211-7

Cleland JG, Freemantle N, Ball SG, Bonser RS, Camici P, Chattopadhyay S, Dutka D, Eastaugh J, Hampton J, Large S, et al. (2003): The heart failure revascularisation trial (HEART): rationale, design and methodology. Eur J Heart Fail $\underline{5}, 295-303$

Cleland JG, Tendera M, Adamus J, Freemantle N, Polonski L, Taylor J; PEP-CHF Investigators (2006): The perindopril in elderly people with chronic heart failure (PEP-CHF) study. Eur Heart J 27, 2338-45

Curtis JP, Selter JG, Wang Y, Rathore SS, Jovin IS, Jadbabaie F, Kosiborod M, Portnay EL, Sokol SI, et al. (2005): The obesity paradox: body mass index and outcomes in patients with heart failure. Arch Intern Med $\underline{165}$, 55-61

Diller PM, Smucker DR, David B, Graham RJ. (1999): Congestive heart failure due to diastolic or systolic dysfunction. Frequency and patient characteristics in an ambulatory setting. Arch Fam Med $\underline{8}, 414-420$

Dolezal T (2006): Imidapril in heart failure. J Renin Angiotensin Aldosterone Syst $\underline{7}, 146-54$ 
Edelmann F, Gelbrich G, Düngen HD, Fröhling S, Wachter R, Stahrenberg R, Binder L, Töpper A, Lashki DJ, Schwarz S, et al. (2011): Exercise training improves exercise capacity and diastolic function in patients with heart failure with preserved ejection fraction: results of the Ex-DHF (Exercise training in Diastolic Heart Failure) pilot study. J Am Coll Cardiol $\underline{58}$, $1780-91$

Edwards NL (2008): The role of hyperuricemia and gout in kidney and cardiovascular disease. Cleve Clin J Med $\underline{75}$, 13-6.

Erentürk S, Yildiz CE, Gulbaran M (2007): Revascularization in patients with severe left ventricular impairment who have ischemic heart disease. Saudi Med J 28, 54-9

ESC=European Society of Cardiology; Heart Failure Association of the ESC (HFA); European Society of Intensive Care Medicine (ESICM), Dickstein K, Cohen-Solal A, Filippatos G, McMurray JJ, Ponikowski P, Poole-Wilson PA, Strömberg A, van Veldhuisen DJ, Atar D, Hoes AW, et al. (2008): ESC guidelines for the diagnosis and treatment of acute and chronic heart failure 2008: the Task Force for the diagnosis and treatment of acute and chronic heart failure 2008 of the European Society of Cardiology. Developed in collaboration with the Heart Failure Association of the ESC (HFA) and endorsed by the European Society of Intensive Care Medicine (ESICM). Eur J Heart Fail 10, 933-989

Fang ZY, Sharman J, Prins JB, Marwick TH (2005): Determinants of exercise capacity in patients with type 2 diabetes. Diabetes Care $\underline{28}, 1643-8$

Fiorina C, Vizzardi E, Lorusso R, Maggio M, De Cicco G, Nodari S, Faggiano P, Dei Cas L (2007): The 6-min walking test early after cardiac surgery. Reference values and the effects of rehabilitation programme. Eur J Cardiothorac Surg $\underline{32}, 724-9$

Flather MD, Shibata MC, Coats AJ, Van Veldhuisen DJ, Parkhomenko A, Borbola J, CohenSolal A, Dumitrascu D, Ferrari R, Lechat P, et al.; SENIORS Investigators (2005): Randomized trial to determine the effect of nebivolol on mortality and cardiovascular hospital admission in elderly patients with heart failure (SENIORS). Eur Heart J $\underline{26}, 215-25$

Ford I, Bezlyak V, Stott DJ, Sattar N, Packard CJ, Perry I, Buckley BM, Jukema JW, de Craen AJ, Westendorp RG, et al. (2009): Reduced glomerular filtration rate and its association with clinical outcome in older patients at risk of vascular events: secondary analysis. PLoS Med $\underline{6}, 77-82$ 
Gallagher MJ, Franklin BA, Ehrman JK, Keteyian SJ, Brawner CA, deJong AT, McCullough PA (2005): Comparative impact of morbid obesity vs heart failure on cardiorespiratory fitness. Chest $\underline{127}, 2197-203$

Guazzi M, Brambilla R, Pontone G, Agostoni P, Guazzi MD (2002): Effect of non-insulindependent diabetes mellitus on pulmonary function and exercise tolerance in chronic congestive heart failure. Am J Cardiol $\underline{89}, 191-7$

Guazzi M, Tumminello G, Matturri M, Guazzi MD (2003): Insulin ameliorates exercise ventilatory efficiency and oxygen uptake in patients with heart failure-type 2 diabetes comorbidity. J Am Coll Cardiol $\underline{42}, 1044-50$

Gustafsson F, Torp-Pedersen C, Seibaek M, Burchardt H, Nielsen OW, Køber L; DIAMOND study group (2006): A history of arterial hypertension does not affect mortality in patients hospitalised with congestive heart failure. Heart $\underline{92}, 1430-3$

Herlitz J, Wikstrand J, Denny M, Fenster P, Heywood T, Masszi G, Rasmussen S, Thorgeirsson G, Wachtell K; MERIT--HF Study Group (2002): Effects of metoprolol CR/XL on mortality and hospitalizations in patients with heart failure and history of hypertension. $\mathrm{J}$ Card Fail $\underline{8}$, 8-14

Herold G: Lipidstoffwechselstörungen; in: Innere Medizin 2008, hrsg. v. Gerd Herold und Mitarbeiter, Eigenverlag Köln 2008; 645-652

Hillege HL, Girbes AR, de Kam PJ, Boomsma F, de Zeeuw D, Charlesworth A, Hampton JR, van Veldhuisen DJ (2000): Renal function, neurohormonal activation, and survival in patients with chronic heart failure. Circulation $\underline{10}, 2203-10$

Hjalmarson A, Goldstein S, Fagerberg B, Wedel H, Waagstein F, Kjekshus J, Wikstrand J, El Allaf D, Vítovec J, Aldershvile J, et al. (2000): Effects of controlled-release metoprolol on total mortality, hospitalizations, and well-being in patients with heart failure: the Metoprolol CR/XL Randomized Intervention Trial in congestive heart failure (MERIT-HF). MERIT-HF Study Group. JAMA 283, 1295-302

Ho KK, Pinsky JL, Kannel WB, Levy D (1993): The epidemiology of heart failure: the Framingham study. J Am Coll Cardiol 22, 6A-13A 
Hobbs FDR (2009): Clinical burden and health service challenges of chronic heart failure.

Eur J Heart Fail Suppl $\underline{8}$, i1-i4

Horwich TB, Fonarow GC, Hamilton MA, MacLellan WR, Borenstein J (2002): Anemia is associated with worse symptoms, greater impairment in functional capacity and a significant increase in mortality in patients with advanced heart failure. J Am Coll Cardiol $\underline{39}, 1780-86$

Horwich TB, Leifer ES, Brawner CA, Fitz-Gerald MB, Fonarow GC; HF-ACTION Investigators (2009): The relationship between body mass index and cardiopulmonary exercise testing in chronic systolic heart failure. Am Heart J $\underline{158}$, S31-6

Ingle L, Reddy P, Clark AL, Cleland JG (2006): Diabetes lowers six-minute walk test performance in heart failure. J Am Coll Cardiol $\underline{47}, 1909-10$

Iribarren C, Karter AJ, Go AS, Ferrara A, Liu JY, Sidney S, Selby JV (2001): Glycemic control and heart failure among adult patients with diabetes. Circulation $\underline{103}, 2668-2673$

Itoh K, Osada N, Inoue K, Samejima H, Seki A, Omiya K, Miyake F (2005): Relationship between exercise intolerance and levels of neurohormonal factors and proinflammatory cytokines in patients with stable chronic heart failure. Int Heart J $\underline{46}, 1049-59$

Jankowska EA, Ponikowska B, Majda J, Zymlinski R, Trzaska M, Reczuch K, BorodulinNadzieja L, Banasiak W, Ponikowski P (2007): Hyperuricaemia predicts poor outcome in patients with mild to moderate chronic heart failure. Int J Cardiol $\underline{115}, 151-5$

Jin B, Luo X, Lin H, Li J, Shi H (2010): A meta-analysis of erythropoiesis-stimulating agents in anaemic patients with chronic heart failure. Eur J Heart Fail 12, 249-53

Kalantar-Zadeh K, Horwich TB, Oreopoulos A, Kovesdy CP, Younessi H, Anker SD, Morley JE (2007): Risk factor paradox in wasting diseases. Curr Opin Clin Nutr Metab Care 10, 43342

Kalra PR, Bolger AP, Francis DP, Genth-Zotz S, Sharma R, Ponikowski PP, Poole-Wilson PA, Coats AJ, Anker SD (2003): Effect of anemia on exercise tolerance in chronic heart failure in men. Am J Cardiol 91, 888-91 
Karapolat H, Eyigor S, Atasever A, Zoghi M, Nalbantgil S, Durmaz B (2008): Effect of dyspnea and clinical variables on the quality of life and functional capacity in patients with chronic obstructive pulmonary disease and congestive heart failure. Chin Med J (Engl) $\underline{121}$, $592-6$

Klein R, Klein BE, Cornoni JC, Maready J, Cassel JC, Tyroler HA (1973): Serum uric acid. Its relationship to coronary heart disease risk factors and cardiovascular disease, Evans County, Georgia. Arch Intern Med 132, 401-10

Kunju SU, Badarudeen S, Schwarz ER (2009): Impact of obesity in patients with congestive heart failure. Rev Cardiovasc Med $\underline{10}, 142-51$

Lam CS, Roger VL, Rodeheffer RJ, Bursi F, Borlaug BA, Ommen SR, Kass DA, Redfield MM (2007): Cardiac structure and ventricular-vascular function in persons with heart failure and preserved ejection fraction from Olmsted County, Minnesota. Circulation $\underline{115}, 1982-90$

Lavie CJ, Milani RV, Ventura HO (2009): Obesity and cardiovascular disease: risk factor, paradox, and impact of weight loss. J Am Coll Cardiol $\underline{53}, 1925-32$

Levy D, Larson MG, Vasan RS (1996): The progression from hypertension to congestive heart failure. JAMA $\underline{275}, 1557-1562$

Leyva F, Chua TP, Anker SD, Coats AJ (1998): Uric acid in chronic heart failure: a measure of the anaerobic threshold. Metabolism $\underline{47}, 1156-9$

Likoff MJ, Chandler SL, Kay HR (1987): Clinical determinants of mortality in chronic congestive heart failure secondary to idiopathic dilated or to ischemic cardiomyopathy. Am J Cardiol $\underline{59}, 634-8$

MacDonald MR, Petrie MC, Hawkins NM, Petrie JR, Fisher M, McKelvie R, Aguilar D, Krum H, McMurray JJ (2008): Diabetes, left ventricular systolic dysfunction, and chronic heart failure. Eur Heart J $\underline{29}$, 1224-40

Mancini DM, Katz SD, Lang CC, LaManca J, Hudaihed A, Androne AS (2003): Effect of erythropoietin on exercise capacity in patients with moderate to severe chronic heart failure. Circulation 107, 294-9 
Massie BM, Carson PE, McMurray JJ, Komajda M, McKelvie R, Zile MR, Anderson S, Donovan M, Iverson E, Staiger C, et al.; I-PRESERVE Investigators (2008): Irbesartan in patients with heart failure and preserved ejection fraction. N Engl J Med $\underline{359}, 2456-67$

Mehra MR, Uber PA, Park MH, Scott RL, Ventura HO, Harris BC, Frohlich ED (2004): Obesity and suppressed B-type natriuretic peptide levels in heart failure. J Am Coll Cardiol $\underline{43}, 1590-5$

Nieuwland W, Berkhuysen MA, van Veldhuisen DJ, van Sonderen E, Viersma JW, Lie KI, Rispens P (1998): Impairment of exercise capacity and peak oxygen consumption in patients with mild left ventricular dysfunction and coronary artery disease. Eur Heart J $\underline{19}, 1688-95$

Nodari S, Metra M, Dei Cas L (2003): Beta-blocker treatment of patients with diastolic heart failure and arterial hypertension. A prospective, randomized, comparison of the long-term effects of atenolol vs. nebivolol. Eur J Heart Fail $\underline{5}, 621-7$

Oreopoulos A, Padwal R, Kalantar-Zadeh K, Fonarow GC, Norris CM, McAlister FA (2008): Body mass index and mortality in heart failure: a meta-analysis. Am Heart J $\underline{156}, 13-22$

Owan TE, Hodge DO, Herges RM, Jacobsen SJ, Roger VL, Redfield MM (2006): Trends in prevalence and outcome of heart failure with preserved ejection fraction. N Engl J Med $\underline{355}$, 251-9

Parameswaran K, Todd DC, Soth M (2006): Altered respiratory physiology in obesity. Can Respir J 13, 203-10

Paulus WJ, Tschöpe C, Sanderson JE, Rusconi C, Flachskampf FA, Rademakers FE, Marino P, Smiseth OA, De Keulenaer G, et al. (2007): How to diagnose diastolic heart failure: a consensus statement on the diagnosis of heart failure with normal left ventricular ejection fraction by the Heart Failure and Echocardiography Associations of the European Society of Cardiology. Eur Heart J $\underline{28}, 2539-50$

Resl M, Hülsmann M, Pacher R, Clodi M (2009): Heart failure in diabetes. Wien Med Wochenschr $\underline{159}, 134-40$

Sietsema KE, Amato A, Adler SG, Brass EP (2004): Exercise capacity as a predictor of survival among ambulatory patients with end-stage renal disease. Kidney Int. $\underline{65}, 719-24$ 
Silverberg DS, Wexler D, Sheps D, Blum M, Keren G, Baruch R, Schwartz D, Yachnin T, Steinbruch S, Shapira I, et al. (2001): The effect of correction of mild anemia in severe, resistant congestive heart failure using subcutaneous erythropoietin and intravenous iron: a randomized controlled study. J Am Coll Cardiol $\underline{37}, 1775-80$

Silverberg D, Wexler D, Blum M, Schwartz D, laina A (2004): The association between congestive heart failure and chronic renal disease. Curr Opin Nephrol Hypertens $\underline{13}, 163-70$

Smith GL, Masoudi FA, Vaccarino V, Radford MJ, Krumholz HM (2003): Outcomes in heart failure patients with preserved ejection fraction: mortality, readmission, and functional decline. J Am Coll Cardiol $\underline{41}$, 1510-8

Stassijns G, Lysens R, Decramer M (1996): Peripheral and respiratory muscles in chronic heart failure. Eur Respir J $\underline{9}, 2161-7$

Suskin N, McKelvie RS, Burns RJ, Latini R, Pericak D, Probstfield J, Rouleau JL, Sigouin C, Solymoss CB, Tsuyuki R, et al. (2000): Glucose and insulin abnormalities relate to functional capacity in patients with congestive heart failure. Eur Heart $\mathrm{J} \underline{21}, 1368-75$

Teerlink JR, Goldhaber SZ, Pfeffer MA (1991): An overview of contemporary etiologies of congestive heart failure. Am Heart $\mathrm{J} \underline{121}, 1852-3$

Terziyski KV, Marinov BI, Aliman OI, St Kostianev S (2009): Oxygen uptake efficiency slope and chronotropic incompetence in chronic heart failure and chronic obstructive pulmonary disease. Folia Med (Plovdiv) $\underline{51}, 18-24$

Tibb AS, Ennezat PV, Chen JA, Haider A, Gundewar S, Cotarlan V, Aggarwal VS, Talreja A, Le Jemtel TH (2005): Diabetes lowers aerobic capacity in heart failure. J Am Coll Cardiol $\underline{46}$, $930-1$

Tomita M, Mizuno S, Yamanaka H, Hosoda Y, Sakuma K, Matuoka Y, Odaka M, Yamaguchi M, Yosida H, Morisawa H, et al. (2000): Does hyperuricemia affect mortality? A prospective cohort study of Japanese male workers. J Epidemiol 10, 403-9

Townend JN, Pagano D, Allen SM, Jordan P, Davies MK, Littler WA, Bonser RS (1995): Results of surgical revascularization in ischaemic heart failure without angina. Eur $\mathrm{J}$ Cardiothorac Surg $\underline{9}, 507-13$ 
Vasan RS, Larson MG, Benjamin EJ, Evans JC, Reiss CK, Levy D (1999): Congestive heart failure in subjects with normal versus reduced left ventricular ejection fraction: prevalence and mortality in a population-based cohort. J Am Coll Cardiol $\underline{33}, 1948-55$

Volterrani M, Clark AL, Ludman PF, Swan JW, Adamopoulos S, Piepoli M, Coats AJ (1994): Predictors of exercise capacity in chronic heart failure. Eur Heart J $\underline{15}, 801-9$

Wang TD, Lee CM, Wu CC, Lee TM, Chen WJ, Chen MF, Liau CS, Sung FC, Lee YT (1999): The effects of dyslipidemia on left ventricular systolic function in patients with stable angina pectoris. Atherosclerosis $\underline{146}, 117-24$

Wexler D, Silverberg D, Blum M, Sheps D, Keren G, Wollman Y, Schwartz D, Iaina A (2005): Anaemia as a contributor to morbidity and mortality in congestive heart failure. Nephrol Dial Transplant 20, vii11-5.

Wilson JR, Mancini DM (1993): Factors contributing to the exercise limitation of heart failure. J Am Coll Cardiol 22, 93A-98A

Yusuf S, Pfeffer MA, Swedberg K, Granger CB, Held P, McMurray JJ, Michelson EL, Olofsson B, Ostergren J; CHARM Investigators and Committees (2003): Effects of candesartan in patients with chronic heart failure and preserved left-ventricular ejection fraction: the CHARM-Preserved Trial. Lancet $\underline{362}, 777-81$

Zannad F, McMurray JJ, Krum H, van Veldhuisen DJ, Swedberg K, Shi H, Vincent J, Pocock SJ, Pitt B; EMPHASIS-HF Study Group (2011): Eplerenone in patients with systolic heart failure and mild symptoms. N Engl J Med $\underline{364}, 11-21$

Zile MR, Brutsaert DL (2002 a): New concepts in diastolic dysfunction and diastolic heart failure: Part I: diagnosis, prognosis, and measurements of diastolic function. Circulation $\underline{105}$, 1387-93

Zile MR, Brutsaert DL (2002 b): New concepts in diastolic dysfunction and diastolic heart failure: Part II: causal mechanisms and treatment. Circulation $\underline{105}, 1503-8$ 


\section{Danksagung}

Zunächst möchte ich mich bei Herrn Prof. Dr. G. Hasenfuß, dem Direktor der Abteilung Kardiologie und Pneumologie der Universitätsmedizin Göttingen bedanken, der mir die Möglichkeit gab, meine Dissertation in seiner Abteilung zu erstellen.

Besonderer Dank gilt Herrn Dr. med. F. Edelmann, meinem Betreuer, für sein außerordentliches Engagement und die enorme Unterstützung besonders während der Zeit der Auswertung der Ergebnisse und der Niederschrift. Seine Betreuung sowie seine unermüdliche Geduld und Motivation zu jeder Phase der Arbeit haben den größten Anteil am Zustandekommen dieser Dissertation.

Nicht weniger Dank gebührt meinem Doktorvater Herrn PD Dr. med. R. Wachter für die raschen Rückmeldungen, die fachliche Bewertung und die wohlwollende Begleitung meines Promotionsverfahrens.

Weiterhin danke ich allen Mitarbeitern, Doktoranden und Doktorandinnen für die kollegiale, nette und unkomplizierte Zusammenarbeit sowie den zahlreichen Patienten, die an den verschiedenen Teilprojekten teilgenommen haben.

Mein Dank gilt weiterhin meinen Eltern Kerstin und Gerhard Durstewitz für ihre Hilfe bei der Erstellung und Korrektur der äußeren Form dieser Arbeit sowie für ihre unentwegte tatkräftige, moralische und finanzielle Unterstützung.

Schließlich möchte ich mich bei Fabian Nolte für die ausdauernde und gewissenhafte formale Korrektur dieser Arbeit sowie für die stetige Motivation und seinen fortwährenden Rückhalt bedanken. 


\section{Lebenslauf}

Am 26. Mai 1986 wurde ich, Kathleen Durstewitz, als Tochter von Gerhard Durstewitz und Kerstin Durstewitz (geb. Michel) in Heiligenstadt geboren. Nach der Einschulung im Jahr 1992 besuchte ich bis 1996 die Grundschule in Birkenfelde. Danach wechselte ich auf das katholische Gymnasium „Bergschule St. Elisabeth“ in Heilbad Heiligenstadt, an dem ich im Jahr 2004 die Allgemeine Hochschulreife erwarb.

Im Rahmen eines Freiwilligen Sozialen Jahres arbeitete ich im Anschluss an die Schulzeit bis März 2005 im Evangelischen Krankenhaus Weende in Göttingen. Zum Sommersemester 2005 nahm ich mein Studium der Humanmedizin an der Georg-August-Universität Göttingen auf und schloss im März 2007 den Ersten Abschnitt der Ärztlichen Prüfung erfolgreich ab. In meiner insgesamt viermonatigen Famulatur durchlief ich die Fächer Chirurgie, Nephrologie, Gynäkologie/Geburtshilfe und Kardiologie. Im August 2010 trat ich das Praktische Jahr im Bereich Chirurgie in der Schweiz an und absolvierte anschließend bis März 2011 die Tertiale in den Fächern Innere Medizin und Anästhesiologie in Göttingen. Im Oktober 2011 schloss ich mein Humanmedizinstudium mit dem Zweiten Abschnitt der Ärztlichen Prüfung erfolgreich ab.

Die hier vorliegende Dissertation begann ich im Jahr 2009 in der Abteilung Kardiologie und Pneumologie an der Universität Göttingen unter der Doktorvaterschaft von Herrn PD Dr. med. Rolf Wachter und der Betreuung von Herrn Dr. med. Frank Edelmann. 